NEW GRADUATES' VALUES AND THE EFFECT OF A NURSE TRANSITION PROGRAM ON NEOPHYTE STAFF NURSES' CLINICAL COMPETENCY, ROLE ADJUSTMENT, JOB SATISFACTION, LENGTH OF STAY, AND TURNOVER

Lillian Jane Currie

Charlottesville, Virginia

B.S.N., University of Virginia, 1976

M.S.N., George Mason University, 1986

A Dissertation Presented to the Graduate

Faculty of the University of Virginia

in Candidacy for the Degree of

Doctor of Philosophy

School of Nursing

University of Virginia

August 1994

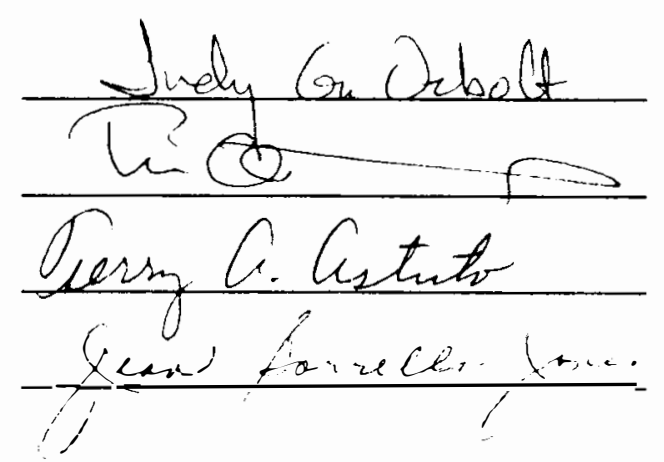


ii

- Copyright by

Lillian Jane Curie

All rights reserved August 1994 


\title{
New Graduates' Values and The Effect of a Nurse Transition Program on Neophyte Staff Nurses' Clinical Competency, Role Adjustment, Job Satisfaction, Length of Stay, and Turnover
}

\begin{abstract}
Persistent fluctuations in the supply of new graduate nurses into the labor force, concomitant with an ever increasing demand for experienced nurses in a variety of settings, has prompted hospital nursing administrators to increase their departments' attention on promoting staff nurse retention and reducing turnover. Specifically, in an attempt to address the causes and consequences of new graduates' experience of "reality shock" during their first year of employment, many institutions have implemented transition programs to aid in bridging the gap between education and service. Using longitudinal data from a convenience sample of 890 new graduate staff nurses, this study addressed the following questions: what is the effect of a nurse transition program on neophyte staff nurses' clinical competency, role adjustment, job satisfaction, length of stay, and turnover?; and, what are the nature and discriminating characteristics of neophyte nurses' professional and role-oriented values? Repeated measures analyses of variance (RM-ANOVAs) indicated that the new graduates' clinical competency significantly increased over time, as did their familiarity with work demands and the hospital environment. In addition, subjects demonstrated a significant increase in their level of job satisfaction concerning involvement. However, nurses' scores significantly declined over time with regard to their perceptions of the actual practice of professional nursing. Subjects' length of stay was slightly higher than the average length of stay of new graduates at the hospital study site prior to implementation of the transition program; and, compared to the pre-program neophyte turnover rate, nurses in the study had a
\end{abstract}


substantially lower rate of attrition. There were no significant differences between new graduates who stayed beyond 15 months after hire (stayers) and those who terminated employment within 15 months (leavers) on the outcome variables of clinical competency, role adjustment, and job satisfaction. Investigation of the professional and role-oriented values possessed by the new graduate nurses revealed that their strongest values were associated with dimensions of personal and professional benevolence, growth, and opportunity, and working effectively with others and within the hospital system. The results of RM-ANOVAs indicated that the new graduates' values significantly decreased over time with respect to intrinsic and extrinsic rewards and stress and autonomy. However, subjects' values related to organizational relationships and roles, as well as professional development and clinical practice, increased significantly over time. There were no significant differences between stayers and leavers with respect to their professional and role-oriented values.

The results of this study suggest that the transition program was effective in promoting the new graduates' increase in clinical proficiency and service role functioning, and in sustaining a positive degree of job satisfaction. In addition, the study's findings suggest that the transition program was effective in increasing the length of stay and lowering the turnover rates for new graduates at the study hospital. However, the study does not provide evidence that the transition program was effective in promoting the new graduates' professional role development. Rather, the results indicate that either the nurses' declining sense of professionalism was moderated by an increasing service orientation; or, that the consequences of their increasingly negative perceptions 
of professional nursing in general, and their professional role in particular, were not apparent within the time frame of data collection. Nevertheless, the results of this study have important implications for nursing education, practice, management, research and knowledge development.

Recommendations are made for additional analyses of the data in order to provide more in depth description of the relationships between neophyte staff nurses and their clinical skill level, role functioning, perceptions of professional nursing, and job satisfaction. Future research should also focus on the outcomes associated with the organizational structures currently advocated in the nursing and non-nursing organizational literature which may further enhance the clinical functioning, role adaptation, and job satisfaction of new graduate staff nurses in hospital settings. Furthermore, it is recommended that future research related to organizational factors include data that is collected through naturalistic methods of inquiry, as well as information on social and economic factors. Research of this type is needed in order to provide critical perspectives to interpretation of the such studies' findings and to assist in the formulation of more conclusive results with regard to transition programs and nurses' professional and role-oriented values. 


\section{UNIVERSITY OF VIRGINIA \\ SCHOOL OF NURSING}

Ph.D. Program

\section{DISSERTATION APPROVAL SHEET}

Formal approval is hereby given to this submitted dissertation by

Lillian Jane Currie on New Graduates' Values and The Effect of a Nurse Transition Program on Neophyte Staff Nurses' Clinical Competency, Role Adjustment, Job Satisfaction, Length of Stay, and Turnover.

July 12,1994

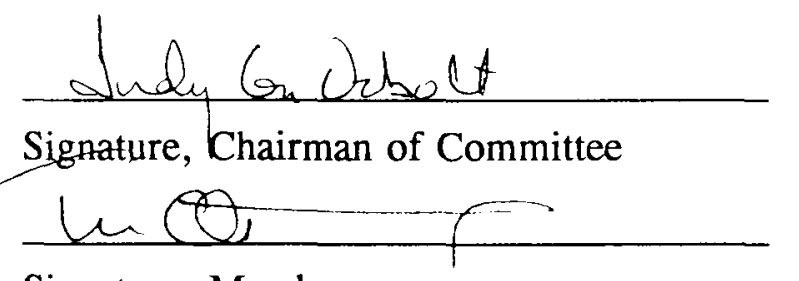

Signature, Member

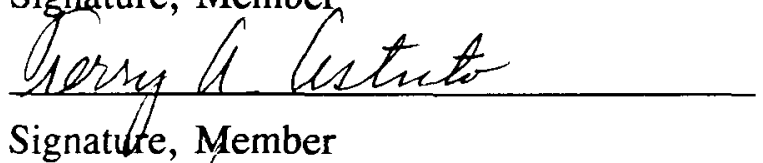

Signatute, Member
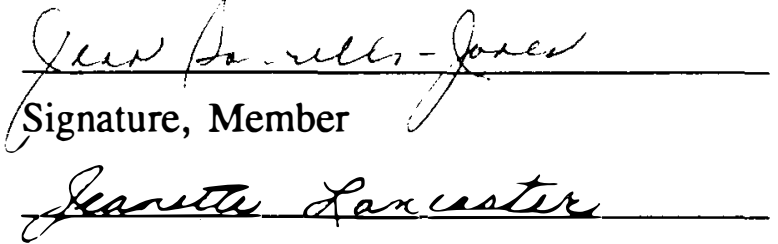

Signature

Dean, School of Nursing 
TABLE OF CONTENTS

Page

$\begin{array}{ll}\text { COPYRIGHT } & \text { ii }\end{array}$

ABSTRACT

SIGNATURE PAGE vi vi

LIST OF TABLES $\quad$ ix

DEDICATION $\quad$ xi

Chapter

I INTRODUCTION

Overview 2

Theoretical Framework $\quad 5$

Organizational Theory Perspectives $\quad 7$

Nature of the Problem 10

Problem Statement 12

Purpose $\quad 12$

Research Questions $\quad 13$

Significance $\quad 14$

$\begin{array}{ll}\text { Summary } & 17\end{array}$

II LITERATURE REVIEW $\quad 18$

$\begin{array}{ll}\text { Theoretical Framework } & 18\end{array}$

Neophyte Nurse Transition Programs $\quad 27$

Organizational Theory Perspectives on Employee Values 41

Nursing Literature and Research on Employee Values 50

Summary $\quad 55$

III METHODOLOGY $\quad 58$

Design $\quad 58$

Setting and Sample $\quad 60$

Description of the Independent Variable $\quad 61$

Dependent Measures 65

Data Analysis Procedures $\quad 69$ 
Limitations

Summary

IV RESULTS

Sample Characteristics

Research Question 1

Research Question 2

Research Question 3

Research Question 4

Research Question 5

Research Question 6

Research Question 7

Summary

V DISCUSSION

Overview

Interpretations

Strengths and Limitations 126

Implications

Recommendations

133

Summary

APPENDIX A. Consent Form 


\section{LIST OF TABLES}

Tables

Page

1 Transition Programs' Duration and Content

2 Demographic Characteristics for Each Panel Year and the Aggregate Sample

3 Significance of Differences on the Demographic Variables for the Panel Groups

4 Measures of Central Tendency and Dispersion and Results of Cross-Sectional Analyses on the Outcome Variables for the Panel Groups at the First Time Point

5 Demographic Characteristics and Significance of Differences for Stayers and Leavers

6 Measures of Central Tendency and Dispersion on the Outcome Variables for the Panel Groups and the Aggregate Sample at Each Time Point

7 RM-ANOVAs on the Outcome Variables for the Panel Year Groups

8 One-Sample RM-ANOVAs for Each Panel Group on the Outcome Variables

9 Length of Stay for Each Panel Year and the Aggregate Sample

10 Turnover Rates (TOR) for Each Panel Year and the Aggregate Sample

11 Measures of Central Tendency and Dispersion on the Outcome Variables for Stayers and Leavers at Each Time Point

12 RM-ANOVAs on the Outcome Variables for Stayers and Leavers 
14 RFS Factor Structure

15 Measures of Central Tendency and Dispersion for the NCS and RFS Factors at the Three Time Points

16 RM-ANOVAs on the NCS and RFS Factors

17 Measures of Central Tendency and Dispersion on the NCS and RFS Factors for Stayers and Leavers at the Three Time Points

18 RM-ANOVAs on the NCS and RFS Factors for Stayers and Leavers 
To my mother and father, Adele and James Currie, whose love, support, and guidance throughout my life have sustained me, strengthened me, and revealed to me the way to the better life.

And, to Vicki. 


\section{Chapter I}

\section{INTRODUCTION}

Prior to the more recent concem about tumover among hospital staff nurses in general, many nurse administrators and scholars focused much attention on the causes of, and solutions for, new graduate nurses' tumover in particular. This focus was stimulated by the theoretical work advanced in the 1970's by Kramer, Schmalenberg, and Benner \& Benner regarding the values and role conflict new nurses experienced upon entry in the work setting. In addition, this focus was sustained as new graduate transition programs were implemented in hospitals across the country in response to the belief that such programs would prevent or ameliorate this conflict. Unfortunately, there has been little systematic and objective evaluation as to the outcomes and benefits of these transition programs.

Using longitudinal data, this study examined the effect of a nurse transition program on neophyte staff nurses' clinical competency, role adjustment, job satisfaction, length of stay, and tumover. In addition, this study sought to identify the professional and role-oriented values new nurses possessed upon initial employment, to determine whether these values changed over time, and to discern whether differing values affected the nurses' length of stay.

This chapter provides an overview of the issues surrounding staff nurse turnover and the problems of the neophyte nurse's entry into professional practice. In addition, a description of the study's theoretical framework, statement of the problem, and 
delineation of the study's research questions are provided, followed by a discussion of the study's significance.

\section{Overview}

Persistent fluctuations in the supply of new graduates into the labor force, concomitant with an ever increasing demand for nurses in a variety of settings, has prompted hospital nursing administrators to increase their departments' attention on promoting staff nurse retention and reducing turnover. In addition, health care institutions have become keenly interested in identifying the causes of staff nurse turnover due to major changes in the structure of the health care delivery and increasing pressure to reduce costs. Furthermore, the negative effects of poor turnover and retention rates among staff nurses in hospitals are believed to be detrimental to quality patient care, industry competitiveness, personnel productivity, and professional enhancement (Alexander, 1989; Bame, 1993; Cavanagh, 1989; Garner, Smith, \& Piland, 1990; Hinshaw, Smeltzer, \& Atwood, 1987; Porter-O'Grady, 1986; Weisman, Alexander, \& Chase, 1981). Many believe that proper management of nursing employment stability will result in increased quality of patient care and increased patient and family satisfaction with care, as well as improved financial health and long-term survival of health care institutions (Bame, 1993; Hinshaw et al, 1987; Kramer, 1985; Pooyan, Eberhardt, \& Szigeti, 1990; Whaley, Young, Adams, \& Biordi, 1989).

Budgetary costs related to nurse turnover are perhaps of greatest concern to hospital and nursing administrators. In the last twenty years, estimates of annual nursing turnover have ranged from $30 \%$ to $70 \%$ (Blegen \& Mueller, 1987; McCloskey, 1974; 
Prescott \& Bowen, 1987; Weisman et al., 1981), with the current national average attrition rate ranging between 15 and 32 percent (Bame, 1993; Cowart \& Serow, 1992; Health Care Advisory Board, 1987; Vanevenhoven, Stull, \& Pinkerton, 1988; Virginia Hospital Association (VHA), 1991). Despite this overall decline, nursing tumover rates have been two to three times higher than rates for professionals of comparable education and gender (Bame, 1993). In addition, within this turnover cohort, nurses recruited in the last six months to one year are the most likely to resign; and in general, nurses average 2.3 years in their first hospital job (Health Care Advisory Board, 1987). Similarly, in a recent survey by the Virginia Hospital Association regarding the longevity of full-time registered nurses on hospital staffs, $14.7 \%$ of the nurses stayed less than one year and $13.6 \%$ stayed one to two years; thus, slightly more than $28 \%$ of the nurses resigned within two years of initial employment (VHA, 1991).

Despite the decline in tumover rates per agency in recent years (Bame, 1993; Hinshaw et al., 1987), the costs associated with turnover continue to be problematic for hospitals. The cost of recruiting and orienting a single registered nurse (RN) has been cited as ranging from $\$ 2,000$ to $\$ 5,000$ (Bame, 1993; Cowart \& Serow, 1992; Health Care Advisory Board, 1987; Hinshaw et al., 1987; Prestholdt, Lane, \& Mathews, 1988; Seybolt, 1986), while total replacement costs for one full-time $\mathrm{RN}$ have been estimated to range from $\$ 7000$ to $\$ 20,000$ (Stratton, Dunkin, Juhl, Ludtke, \& Geller, 1991; Vanevenhoven et al., 1988). According to a report by the Health Care Advisory Board (1987), replacement total-cost estimates ranging from $\$ 2,600$ to $\$ 17,500$ are based on the additive costs of recruitment $(\$ 800-\$ 5,000)$, orientation $(\$ 1,800-\$ 3,000)$, productivity 
loss $(\$ 0-\$ 2,500)$, and use of supplemental nurses $(\$ 0-\$ 7,000)$. And, in a study of four acute care hospitals in the southeast, Jones (1990) reported total turnover cost per RN to be from $\$ 6,886$ to $\$ 15,152$, with an average cost of $\$ 10,198$. Jones (1992) subsequently updated these cost data to reflect the effects of inflation rates and shifting dollar values: the previous mean tumover cost of $\$ 10,198$ was calculated to be equivalent to $\$ 12,147$. Given these turnover and cost estimates, one can easily determine the possibility of a hospital experiencing hundreds of thousands of dollars per year in turnover expenses (Cowart \& Serow, 1992; Johnston, 1991; Stratton, et al., 1991).

Although turnover of staff nurses in general has elicited much concern and attention, the tumover of new graduate nurses in particular has received substantial consideration and study. Tumover rates for new graduate, or neophyte, nurses attracted serious attention when it was initially reported in the 1970's to be approximately $50 \%$, and as high as $61 \%$ (Benner \& Benner, 1979; Kramer \& Baker, 1971; Kramer, 1974; McCloskey, 1974). In addition, new graduates' average length of stay in their first position after graduation was six months (Benner, 1974; Kramer \& Schmalenberg, 1978; McCloskey, 1974). Not only did new graduates frequently resign during the orientation period or shortly thereafter, but also according to a National League for Nursing (NLN) study, at 6 to 8 months after graduation, 25.3 percent of newly licensed RNs had worked for 2 or more institutions since graduation (NLN, 1979).

Recent reports also reveal similar statistics. According to the Health Care Advisory Board (1987), the average tenure of new graduates in their first hospital job was 13 months for diploma and associate degree nurses and 8.3 months for baccalaureate 
nurses. Coeling (1990), cites a 1989 study by the NLN which indicated that $24 \%$ of new nursing graduates seek job changes six to nine months after passing their professional licensure exam.

\section{Theoretical Framework}

The most dominant theory to emerge that attempted to explain the phenomenon of new graduate turnover was the notion that neophyte nurses experienced specific problems as they attempted to make the transition from nursing student to staff nurse. Drawing primarily from the sociology literature on professional-bureaucratic role conflict, it was proposed that this difficult passage resulted from the discrepancy in values and goals between nursing education and nursing service (Benner \& Benner, 1979; Kramer, 1968, 1974; Kramer \& Schmalenberg, 1977, 1978). In general, it was noted that the lack of attention to the role transition from student to graduate nurse, with its concomitant set of expectations and responsibilities, led to inadequate performance in the professional role, a sense of dissatisfaction and powerlessness in the nurse's work, and thus a resulting high turnover rate. The neophyte's feelings of disillusionment, frustration, and lack of self-assurance were perceived by most new graduates as a crisis and their response to this crisis has become known in nursing as "Reality Shock". According to Kramer (1974), reality shock is "the specific shock-like reactions of new workers when they find themselves in a work situation for which they have spent several years preparing and for which they thought they were going to be prepared, and then suddenly find they are not" (p. vii-viii). 
Specifically, Kramer $(1968,1974)$ theorized that the first job in a hospital setting was often marked by dramatically conflicting value systems, namely, the idealism of preservice education and the reality of nursing service. In school, nursing students were socialized to a professional model of nursing practice which included a whole-task system of providing care, and the use of judgment, autonomy, knowledge, and decision making. After graduation, a part-task system of care dominated in the work world due to hospitals' emphasis on the bureaucratic characteristics of efficiency, organization, responsibility, and cooperation. Thus, upon entering the work world, the new graduate experienced reality shock because of these unexpected, abrupt changes in values, norms, rewards, and sanctions. In order to both cope with and adapt to these changes, neophyte nurses typically respond by either increasing their loyalty to bureaucratic values concomitant with a decrease in professional values, or by maintaining their professional values and ignoring bureaucratic role influences. According to Kramer (1974), either of these responses eventually leads to increasing job frustration and dissatisfaction, and results in either voluntary or involuntary termination of employment with the hospital.

Kramer \& Schmalenberg (1978) described new graduates who successfully resolve these value and role conflicts as "bicultural", that is, they leamed to integrate school and work values and roles in meaningful, useful ways. Such nurses were able to practice professionally in a bureaucratic organization without undue amounts of role dissatisfaction which was determined to lead to a high incidence of turnover, frequent position changes with the same institution, and exodus from nursing altogether. These authors advocated the implementation of "bicultural training programs" as aids in 
facilitating the role adjustment of new graduates. Such programs aimed to help neophyte nurses achieve competence in the new work subculture without rejecting the values incorporated from nursing school.

The notion of mismatched expectations between education and service was further extended by Benner \& Benner (1979) who identified the conflicting views that nurse educators and nurse employers have about the work role and competency of the new graduate nurse. Areas of significant discrepancy existed between these three groups with regard not only to their values but also to their perceptions of the clinical competencies possessed and required upon entry into hospital nursing practice. Based on these discrepancies, these authors suggested that both nursing service and nursing education have a responsibility to prevent transition problems for the new graduate. Thus, they called for collaboration between service and education regarding curriculum planning, more realistic student clinical practice experiences, and the implementation of internship programs and longer orientation periods in order to enhance the acquisition of a realistic set of basic nursing skills.

\section{Organizational Theory Perspectives}

Since the time that Kramer and her colleagues initially expressed concern about the professional-bureaucratic role conflicts in nursing, attention to employee values and roles has become a prevalent topic in the organizational literature. In particular, this body of literature focuses on employees' values and roles from the perspective of organizational culture, conflict, excellence, and structure. 
According to Raelin (1991), a conflict in values, roles, and expectations between professionals and managers is at the heart of the professional's difficulties in integrating into the bureaucracy of organizations. This conflict, which the author describes as a cultural clash, arises from the fundamentally different educational experiences and early socialization processes that each group undergoes. The clash between these two groups is manifested in managers' expectation that professionals, as employees, will conform to organizational policies and procedures and abide by managers' directives. Professionals, on the other hand, prefer to align themselves with the standards of their discipline and the evaluation of their colleagues and associations. This alignment reduces their commitment to organizational exigencies and fosters, at best, marginal organizational loyalty. As a result of this conflict, professionals' application of their knowledge and skills is thwarted and organizational goals are difficult to achieve. In many cases, especially those involving novice professionals, the professional's frustration and inability to align himself with the organization leads to premature employment termination initiated by either the professional or the organization.

The belief that a discrepancy in values and expectations among individuals and/or groups in organizations is inimical to the accomplishment of goals, and hence organizational growth, effectiveness, and success is embraced by others as well (Grey \& Gelfond, 1990; Kennerley, 1992; Liedtka, 1992; Posner, Kouzes, \& Schmidt, 1985; Schaef \& Fassel, 1988; Shockley-Zalabak \& Morley, 1989; Ullman, 1987). Depree (1989) describes his belief in the establishment of a covenantal relationship between employer and employee that is based on shared values and meanings; genuine caring for, 
support of, and attention to the intrinsic needs and values of employees; and real and meaningful participation in and ownership of the creation of work goals and processes. Gardner (1990), Kanter (1983), and Deal \& Kennedy (1982) point out that the most successful organizations are those whose leaders have created a shared vision with workers based on the identification of common values and goals.

Senge (1990) also prescribes the development of a shared vision as an essential ingredient for creating an innovative, leaming, and thus successful, organization. Shared vision within an organization produces a common identity and destiny. At the root of an organization's shared vision are individuals' ability and freedom to identify and express their personal vision which evolves from their individual core values. Thus, an organization's vision, or mission, should be a reflection of the members' shared values and common aspirations. When organizational goals, processes, and activities evolve from individually held and commonly shared values and visions there is commitment to purpose and methods rather than just compliance to initiatives. According to Senge (1990), the alignment of organizational and workers' values, as well as employees' freedom to function in harmony with their values, is imperative to creating effective work units and producing organizational successes.

The nature of bureaucratically structured organizations as both source and sustenance for values and role conflicts also remains a prevalent theme in current organizational literature. Raelin (1991) describes the culture clash between professionals and managers as "particularly apparent within the confines of bureaucratic organizations" (p.2). Although many would claim that the difficulty in preventing or reducing this 
conflict will abate as organizations take on more humanistic cultures and processes, others would argue to the contrary because of the persistent dominance of the bureaucratic tradition in present day organizations (Clark, 1985; Clark \& Meloy, 1988; Kanter, 1989; Kofman \& Senge, 1993; Morgan, 1986). This tradition, with its reliance on formal structure, authority, efficiency, rationality, and impersonalness, remains deeply ingrained in current conceptions of organization and people in organizations. Consequently, the development of real solutions for the conflicts which professionals and organizations continue to encounter is thwarted. Hence, there are recommendations that extend beyond the notions of values alignment and shared vision to include radically new ways of thinking about and structuring organizations (Clark, 1985; Kofman \& Senge, 1993; Weick, 1979).

\section{Nature of the Problem}

In an attempt to address the causes and consequences associated with high neophyte staff nurse turnover in general, and the occurrence of "reality shock" in particular, nursing departments in hospitals turned to a variety of new organizational interventions. And, specifically in response to the theoretical work of Kramer and her colleagues, a burgeoning body of nursing literature advocated and described the implementation of neophyte nurse transition programs to aid in bridging the gap between education and service (Gibbons \& Lewison, 1980; Health Care Advisory Board, 1987; Kotecki, 1992; Lewison \& Gibbons, 1980; Roell, 1981; Schempp \& Rompre, 1986). Taking various forms and names such as bicultural training programs, preceptorships, and internships, these interventions were proposed to be solutions to the problems of low 
retention and high turnover among neophyte practitioners because they were believed to increase nurses' adjustment, confidence and competence. In addition, such transition programs were believed to increase job satisfaction, reduce dissatisfaction, and be instrumental in recruiting nursing personnel to hospitals. Note, for instance, the following description of ransition programs included in the Health Care Advisory Board's 1987 report:

[Transition programs] are extremely effective in reducing turnover among the high-volatility ranks of first-year nurses...[and are] highly recommended for virtually all Advisory Board members. Several hospitals have reported increases of up to $65 \%$ in new nurse retention after they lengthened and upgraded their orientation programs. An emphasis on practical skills and on-the-job training is recommended to help new RNs cope with their new responsibilities. The best programs also assign new nurses to experienced RNs who can serve as mentors during the often difficult transition from the theoretical world of academia to the realities of patient care. (p. 143).

In response to the plethora of literature advocating increasingly comprehensive and extensive orientation programs, in the last decade or so there has been a proliferation of neophyte nurse transition programs in hospitals across the country. However, the adoption of such designs and their fairly widespread implementation have occurred with little systematic and objective evaluation of their outcomes and effectiveness (Gibbons 
\& Lewison, 1980; Kotecki, 1992; Lewison \& Gibbons, 1980; Roell, 1981; Schempp \& Rompre, 1986).

In addition, as hospital and nurse executives have become engaged in the literature regarding organizational culture and its effects, there has been renewed interest in studying the values orientation of staff nurses and its relationship to employment issues (Coeling, 1990; Curran \& Miller, 1990; del Bueno \& Vincent, 1986; McDaniel \& Stumpf, 1993; Nyberg, 1991; Sovie, 1993). But, only a handful of studies have explored the characteristics and consequences of the professional and role-oriented values possessed by new graduate staff nurses (Ahmadi, Speedling, \& Kuhn-Weissman, 1987; Gardner, 1992; Green, 1988; Speedling, Ahmadi, \& Kuhn-Weissman, 1981).

\section{Problem Statement}

This study sought to answer the following questions: 1) What is the effect of a nurse transition program on neophyte staff nurses' clinical competency, role adjustment, job satisfaction, length of stay, and turnover?; and 2) what are the nature and discriminating characteristics of neophyte nurses' professional and role-oriented values?

\section{Purpose}

The purpose of this study was to assess whether, and to what degree, new graduate nurses' clinical competency, role adjustment, job satisfaction, and length of stay increase, and their rate of turnover decreases, as a result of their participation in a neophyte transition program. In addition, this study sought to determine if, among new graduates participating in the transition program, there are differences with regard to clinical competency, role adjustment, and job satisfaction between those who stay beyond 
15 months after hire, and those who terminate employment within 15 months. Relatedly, this study sought to identify the professional and role-oriented values new nurses possessed upon initial employment, to determine whether these values changed over time, and to discern whether differing values affected the nurses' length of stay.

\section{Research Questions}

Specifically, this study was guided by the following research questions:

1) Do neophyte nurses participating in the transition program demonstrate a significant increase over time in their clinical competency, professional role adjustment, and job satisfaction?

2) Is the length of stay of neophyte nurses participating in the transition program longer than the length of stay of new graduate nurses employed prior to implementation of the transition program?

3) Is the turnover rate of neophyte nurses participating in the transition program less than the turnover rate of new graduate nurses employed prior to implementation of the transition program?

4) Are there significant differences with regard to clinical competency, role adjustment, and job satisfaction between neophyte nurses whose length of stay exceeds 15 months and neophyte nurses who resign within 15 months of employment?

5) What professional and role-oriented values do neophyte nurses possess upon initial employment? 
6) Do neophytes nurses professional and role-oriented values change over time?

7) Are there significant differences with regard to professional and roleoriented values between neophyte nurses whose length of stay exceeds 15 months and neophyte nurses who resign within 15 months of employment?

\section{Significance}

The initial work of Kramer, Schmalenberg, and Benner regarding new graduates' entry into the hospital work force is still relevant in today's environment (Kramer, 1985). Specifically, this original body of literature brought into focus a problem which continues to be problematic. The demand for registered nurses requires continued attention to the issues of staff nurse turnover as the need for RNs is fueled by advances in medical technology, increasingly sophisticated job roles, rising patient acuity, changes in health care delivery structures and financing, and shifting societal demographics (Bame, 1993; Curran \& Miller, 1990; McCloskey \& Grace, 1990; Minnick, Roberts, Curran, \& Ginzberg, 1989). Given that the impact of these issues is felt in all arenas of professional nursing, the significance of this study lies in its potential contributions to nursing education, practice, management, research, and knowledge development.

Nursing education strategies are intimately related to the service sector's prevailing professional and economic issues. The results of this study can further inform nursing educators as to the strategies, activities, and behaviors which may ameliorate the negative consequences associated with students' transition from academe to employment. In addition, because the educational experience is the first phase of the professional's 
socialization process, we must validate the professional and role-oriented values imbued during this process. Furthermore, we must be able to discern whether such values are sustained over time and their relationship to nurses' tenure in the employment setting. Thus, this body of literature and research should be a major consideration for educators as they develop curricula and plan classroom and clinical activities, as well as develop mentoring relationships with students.

From a nursing practice perspective, this study may contribute to further identification and clarification of factors that hinder or promote new graduates' ability to integrate into the employment setting, become successful practitioners, and thus provide quality nursing care to patients and families. Nursing students and novice practitioners may draw upon this body of research to assist them in selecting places of employment which possess the characteristics found to be beneficial in making the transition from student to employee. New employees who are successful in this passage are more likely to fulfill the professional nurse role thus maximizing their individual potential and rendering quality nursing care.

This study's implications and significance to nursing management are multi-fold. First, as increasing numbers of hospitals rely on not only all RN staffs but also higher proportions of experienced nurses, the successful transition from student nurse to experienced practitioner becomes a critical factor in supporting nursing's work force. Assuming that new graduates' perceptions of positive and successful entry into professional practice will enhance their desire and ability to remain in the work force, then the efforts that nursing service place in easing this transition may contribute to 
increasing the supply of experienced nursing staff. In addition, further knowledge of the new nurse's values orientation can assist us in creating organizational processes that are more congruent with the discipline's values and desired roles.

Second, nursing administrators must accept the fact that, as a result of the factors surrounding the demand and supply issue, schools of nursing may continue to do little more than provide a general foundation for practice. Nursing service must ultimately assume responsibility for the performance of the new graduate, since it is the institution that is responsible for the quality of nursing care being provided in hospitals. Recent trends with regard to the implementation of longer and more substantive orientation programs indicate that hospital and nursing service administrators believe these programs enhance such performance (Health Care Advisory Board, 1987; VHA, 1991).

Third, in order to justify the substantial direct and indirect costs incurred by the implementation of nurse transition programs, and in light of their rapid and widespread implementation, the actual outcomes and benefits associated with them nead to be identified. Systematic and objective evaluation of neophyte nurse transition programs can provide nurse administrators with the necessary documentation to allocate human resources and justify cost expenditures, as well as guide policy and program decisions within their institutions. Relatedly, we must add to our knowledge base concerning new practitioners' disciplinary and role values in order to create organizational processes that are non-bureaucratic, more humanistic, professionally congruent, and vision driven.

Finally, the potential contribution of this study to nursing research and knowledge development is also evident. The longitudinal nature of the study's data will promote 
greater confidence in the validity and reliability of its findings, thus providing additional testing of the theoretical framework and extending the body of knowledge which has been articulated to date regarding neophyte nurses' values orientation, transition programs, and new graduate turnover.

\section{Summary}

The human and financial consequences to the patient, the nurse, and the hospital that are associated with staff nurse turnover necessitate that all nursing resources be protected. Nursing researchers and administrators must continue to be concerned with factors that contribute to the employment stability of both experienced and new graduate practitioners. However, studies regarding the turnover of neophyte nurses in particular are especially warranted since new graduates are the original source for the needed supply of experienced practitioners. Furthermore, interventions which support the new graduate's transition into and continued employment with hospitals must be investigated and their outcomes evaluated in order to justify their selection and implementation. This study's investigation of new graduate nurses' professional and role-oriented values, and the effect of a transition program on their clinical competency, role adjustment, job satisfaction, length of stay, and turnover contributes to meeting these research needs. 


\section{Chapter II}

\section{LITERATURE REVIEW}

This chapter provides a review of the theoretical framework and the subsequent research on transition programs as it pertains to neophyte staff nurses' clinical competency, role adjustment, job satisfaction, length of stay, and turnover. In addition, current organizational literature and research concerning employee values are reviewed, followed by a review of the nursing research regarding the characteristics and consequences of new graduates' professional and role-oriented values.

\section{Theoretical Framework}

Drawing from the sociology literature on professional-bureaucratic role conflict, Kramer (1968) examined the effect of exposure to bureaucratic employment on the professional values of new collegiate graduate nurses. Data were collected from a convenience sample of 59 new graduate nurses employed in hospitals determined by the investigator to have bureaucratic organizational structures. Using a longitudinal design, subjects completed questionnaires at graduation and at two time points after employment (i.e., 3 months and 6 months) to measure changes in the neophyte nurses' role conception, role deprivation or disillusionment, and role models. Results of data analysis indicated: an increase in the bureaucratic component and a decrease in the professional component of the nurses' role conception with length of employment; a shift from a school, or professionally-centered, role model to a work-centered role model (i.e., from instructor to head nurse); and a greater degree of role deprivation among subjects retaining instructor role models than among those who changed to work-centered role 
models. New graduate nurses who did not demonstrate an increase in loyalty to bureaucratic values and a decrease in professional values left hospital employment or nursing altogether in significantly greater numbers than those who did. In addition, subjects who left nursing practice, changed jobs because of dissatisfaction, or returned to school, had significantly greater role deprivation scores than subjects who remained in the same job for the six-month period of the study.

A follow-up of these same nurses two years later (Kramer, 1969a) showed an even further decrease in professional role conception, when compared to the six-month post graduation test score, or $\mathrm{t}_{3}$, obtained in the previous study. In addition, the followup study indicated that unless the bureaucratic orientation score increased, the role deprivation scores continued to rise or remained higher than the median of the group.

Based on these initial studies, as well as others which indicated similar findings among experienced nurses (Kramer, 1969b, 1970; Kramer \& Baker, 1971), and new data collected from the diaries of and interviews with new graduates, Kramer (1974) developed a formal description of her theoretical framework in which she linked the phenomena of professional-bureaucratic role conflict and "reality shock". The major tenets of this framework are that the professional orientation provided by college-based schools of nursing causes the role conflict new nurses experience when they begin their practice in bureaucratic work settings; and that the resolution of this conflict between the value systems of the idealism of preservice education versus the reality of nursing service is crucial in determining the nursing practice and employment stability of hospital staff nurses. 
According to Kramer (1974), nursing students are socialized in school to a professional model of nursing practice which includes a whole-task concept of providing care, and relies on the individual's use of her/his judgment, autonomy, knowledge, and decision making skills. This socialization process emphasizes the "shoulds" and "oughts", or "ideals", of nursing practice. Upon entering the work world, however, the new graduate encounters a part-task system of care and a culture which relies on the bureaucratic characteristics of efficiency, organization, responsibility, and cooperation. The hospital socialization process emphasizes the compromises or shortcuts required to get the job done and how values are put to work in the context of less than "ideal" situations, such as staff shortages and emergencies.

The new graduate nurse's confrontation with these conflicting value systems and socialization processes leads to role deprivation, or reality shock. During this phase of adjustment to the work world, the new nurse experiences feelings of disillusionment, betrayal, bitterness, and rejection. Moral outrage or anger, directed at one's former teachers or at the employing organization, is common because the neophyte feels the real world of nursing is not as it should be and not as she was told it would be. This phenomenon is usually manifested in her/his difficulties in caring for groups of patients, relating to work schedules, working with auxiliary workers, coordinating and relating the functions of the various hospital departments, and in physician-nurse conflicts.

As the values- and role-dissonance intensify, the new nurse begins to look for relief from these conflicts and difficulties. In order to both cope with and adapt to the unexpected, abrupt changes in values, norms, rewards, and sanctions, new graduates 
respond by either increasing their loyalty to bureaucratic values concomitant with a decrease in professional values, or by maintaining their professional values and ignoring bureaucratic role influences. In other words, some will over-identify with the work setting's values and processes and become preoccupied with task accomplishment, while also abdicating their professional values; and others will hold tenaciously to their professional values at the exclusion of incorporating any of the work world values. In both scenarios, since these responses are considered maladaptive, the nurse eventually experiences and demonstrates increasing frustration and dissatisfaction, often leading to either voluntary or involuntary termination of employment with the hospital.

In Kramer's (1974) opinion, the more appropriate and desirable response would be for the new graduate to incorporate a blend of the two value systems so that the strengths of both the educational and service cultures are drawn upon. Kramer (1974) labeled this blend "biculturalism" and described it as the ability to view conflict as healthy, creative, and potentially growth producing and to participate in collaborative, problem-solving behavior with others. Such a role transformation ultimately enables the nurse to become a creative change agent for the good of the patient, the hospital, and the profession.

In collaboration with Kramer, and using her theoretical framework, Benner (1974) examined the effect of a pilot "Reality Shock Program" as an ameliorating intervention for the occurrence of reality shock among new graduates during their first few months of hospital employment. The "Reality Shock Program" consisted of six seminars attended by twelve new graduate staff nurses. The main focus of the seminars was to 
assist the nurses to identify and manage the conflicts they were experiencing on their first jobs post graduation. The goal of the program was to facilitate constructive conflict resolution and thus to increase the chances for bicultural adjustment of these new graduates.

The seminars were co-taught by Benner and a staff development instructor and conducted on a weekly basis. The sessions were semi-structured, allowing for both participants' verbalization of feelings and experiences and presentation of didactic material about the culture shock framework. Seminar discussions were tape recorded and the text was analyzed after each seminar in order to plan intervention strategies for the next session. At the end of the seminar series, Benner concluded that the phases and cyclical nature of the socialization processes as outlined in Kramer's theory indeed occurred and that the nurses benefitted from the opportunities to vent and deal with their emotional responses to the conflicts they were experiencing. In addition, Benner concluded that although the nurses' introduction to the culture shock theory material was helpful for conceptually framing and understanding their problems, such material could be put into practice and used for actual conflict resolution only after the nurses' had dealt with their emotional responses to the varying situations. Based on these conclusions, Benner recommended the development of Reality Shock programs by hospital-based staff development departments which would utilize both group learning and self-paced, individualized leaming strategies. Like Kramer, Benner also postulated that such programs would facilitate bicultural role transformation and thus increase new graduates' job satisfaction, productivity, and tenure on the first job. 
Based on this initial, primarily descriptive, research by Kramer and Benner, both authors undertook large-scale research projects to explore further the basic constructs of the "reality shock" theory. The first, directed by Kramer, involved a nationwide study to evaluate, and quantify, the effectiveness of bicultural training programs in producing a more effective role transformation than traditional orientation programs (Kramer \& Schmalenberg, 1978). The study involved the inservice education departments of eight medical centers with approximately 35 to 40 new graduate nurses from each, for a total sample of 307 subjects. Two orientation programs were developed for each hospital: a clinical training program (CTP) simulating traditional orientation programs which emphasized clinical content (control group); and a bicultural training program (BTP), consisting of affective, cognitive, and behavioral components (experimental group). The affective component involved six weekly seminars designed to assist the participants in dealing with work-related conflicts on an emotional level. The cognitive component, five self-instructional modules, covered theoretical material regarding the mediation of conflicting demands and value systems. The behavioral component consisted of several all-day workshops on conflict resolution. These three components were believed to be instrumental to new graduate nurses' development of interpersonal competence which in tum is requisite for achieving bicultural role transformation.

Subjects were randomly assigned to either the control or experimental group according to their nursing unit and completed questionnaires pertaining to the dependent measures at the beginning of their orientation period, and again at nine and twelve months after employment. Due to subject attrition, data analysis was conducted on a 
final sample of 237 , with 124 subjects in the control group and 113 in the experimental group. The findings revealed that in comparison to new graduates who participated in the CTP, new graduates who participated in the BTP: retained higher professional role conceptions; selected more bicultural role behavior choices; scored higher on empathy for others' viewpoints; reported more frequent and more effective change-agent activity; received higher performance ratings; and reported fewer terminations in employment ( $10 \%$ versus $40 \%)$. In contrast to these results, there were no significant differences between the two groups with regard to their degree of role deprivation, self-actualization and self-esteem scores, and valuation of conflict. Kramer \& Schmalenberg (1978) concluded that the bicultural training programs were effective in helping new graduate nurses': maintain their whole-task conceptions of patient care; choose behavioral options that reflected concern for the whole-task system of work as well as judicious regard for the part-task system; operationalize their school ideals and values as change agents; perform at a higher level with regard to clinical competency; and extend their tenure of employment.

The other major research project stimulated by the reality shock framework, and directed by Benner, involved description and evaluation of the clinical competencies and work-entry experiences of new graduate nurses in hospital settings (Benner \& Benner, 1979). Written surveys were obtained from three groups: 160 new graduates, including all 1974 graduates of nursing programs in the two counties where the study was conducted plus a random sample of staff nurses from the 32 acute care hospitals in the area; 312 hospital nursing service and inservice directors, representing all the local 
hospitals; and 29 nurse educators, representing the nursing schools from which the new graduate subjects were drawn. The surveys required respondents to select the level of competency that a new nurse should possess, both ideally and in reality, for performing 112 nursing skills and activities. Developed by the authors, the 112 items reportedly reflected three different areas of beginning nursing practice: clinical judgment, technical skills, and work-role skills. For each item, one of five competency levels could be chosen: Complete Mastery; Competency; Safe but Practice Needed; Supervision Needed; and Supervision and Instruction Needed. In addition, focused interviews and open-ended survey questions were utilized in order to identify similar and dissimilar values held by the three groups.

The major focus of the data analysis involved comparisons between the respondent-identified ideal performance standards and the respondent-observed real performance. With regard to ideal performance, new graduates felt they should have complete mastery of or competence in $59 \%$ of the skills listed, whereas nurse educators and nursing service personnel selected these levels of competency for only $35 \%$ and $21 \%$ of the items, respectively. For those skills requiring supervision or supervision and instruction, new graduates selected only $3 \%$ of the items, compared to educators' $14 \%$ and service personnel's $10 \%$.

With regard to real, or observed, performance, new graduates judged themselves masterful or competent on $18 \%$ of the items, while educators reported new graduates to have actual mastery or competence on $9 \%$ of the skills, and nursing service respondents reported only $3 \%$ of the nurses to possess these levels of competency. Such differences 
between the three groups are noted again pertaining to the percentage of skills requiring supervision or supervision and instruction: new graduates felt they needed such direction and assistance on only $23 \%$ of the activities, versus $30 \%$ and $80 \%$ reported by educators and nursing service personnel, respectively.

These discrepancies between the ideal and real competency expectations were further analyzed using one-way analysis of variance which showed statistically significant differences between the three groups on 104 of the 112 items, with the greatest difference occurring between the new graduates and nursing service personnel. Since the nursing service respondents had significantly higher discrepancy scores than did new graduates on most of the items (92) and significantly higher scores than did educators on more than half of the items (64), the authors were unable to conclude that the major area of discrepant evaluation involved any one category of technical skills, clinical judgment, or work-role items. However, the eight items with no significant difference were all skills requiring clinical judgment and all three groups had lower expectations in terms of competency and mastery on these items.

Based on these findings, the authors concluded that although all three groups perceived large gaps between their ideal and observed level of new graduate performance, the neophyte nurses reported the highest aspirations for competence. They also considered themselves to be more competent than educators or nursing service personnel considered them to be. Nursing service respondents not only had lower ideal performance standards for new graduates than did educators or new nurses, but also had extremely low expectations of new graduates' actual competency levels. Furthermore, 
the authors noted that nursing service respondents rated new graduates on a par with auxiliary workers in terms of the skills they thought new graduates could perform safely for patients without supervision. Finally, in arguing that such mismatched expectations are sufficient to cause significant conflict between neophyte nurses and nursing service personnel, the authors advocated the implementation of internships programs and longer and more substantative orientations for new graduates, noting also that such recommendations were heavily favored by the majority of the respondents in the study.

\section{Neophyte Nurse Transition Programs}

Although a variety of types of transition programs for the neophyte nurse have been reported in the literature, the most widely cited is the internship program. Within the literature on internship programs, there are also tremendous differences across institutions regarding program content, structure, duration, criteria for participation, and evaluation methods. In general, however, these programs are defined as transitional strategies for new graduates that are distinguished by the organization from traditional orientation programs by virtue of their longer duration, comprehensiveness, closer supervision, and increased didactic instruction.

The initial impetus and ongoing objectives of each program described in the literature are identified in similar terms. In general, the desire to "bridge the gap" between student and professional, to provide a "transitional experience", and to meet through a formal programmatic effort the previously unmet needs of both new graduate and service personnel are commonly cited as reasons for program implementation (Dear, Celentano, Weisman \& Keen, 1982; Health Care Advisory Board, 1987; Martel \& 
Edmunds, 1972; Weiss \& Ramsey, 1977). More specifically, the literature reports that transition/internship programs have been instituted by hospitals in response to the following problems: (1) inadequacy of traditional hospital orientation programs to prepare new graduate nurses to perform at acceptable levels (Marchette \& Merker, 1985; Mims, 1984; Sams, Baxter \& Palmer-Smith, 1990); (2) job dissatisfaction, feelings of powerlessness, and high job turnover with its concomitant costs (Aldrich, 1988; Hunter, Pollman \& Moore, 1990; May, Minehan \& Deluty, 1981; Mims, 1984); and (3) difficulty in recruiting nursing personnel (Fleming, Woodcock \& Boyd, 1975; Hartshorn, 1992; Kasprisin \& Young, 1985; Mims, 1984; Ressler, Kruger \& Herb, 1991).

Table 1 provides a detailed description of the transition programs reviewed in this chapter. In general, however, the programs revealed the following characteristics:

1) type of program - eight referred to "Internship" programs in general medical-surgical areas, two described "Internship" programs in critical care units, three labeled their programs "Bicultural" training, and one referred to a "Transition" program;

2) program duration - ranged from six weeks to 12 months, with the majority reporting to be six months in length.;

3) intern educational preparation - ranged from programs for participants with BSN, ADN, and Diploma degrees to those for BSN graduates only, with the majority of programs involving the former;

4) use of preceptors - eight of the 14 programs utilized preceptors, five did not, and one did not specify; 
Table 1

Transition Programs' Duration and Content

\begin{tabular}{lll}
\hline Author(s) & Duration & Content \\
\hline
\end{tabular}

Weiss \& Ramsey 4 months
(1977)

Gibbons \& Lewison (1980)

Holloran, Mishkin

\& Hanson (1980)

Hollefreund, Mooney, 6 weeks Moore \& Jersan

(1981)

May, Minehan \& Deluty (1981)

Dear, Celentano, Weisman \& Keen (1982)
6 months Classes, conferences, seminars, and case presentations 8 hours per week.

5 months Six weekly seminars dealing with school to work values conflict (using Kramer's affective and cognitive components); 3 all-day workshops for graduates and head nurses on feedback and conflict resolution (using Kramer's behavioral component).

Five weekly seminars dealing with school to work values conflict (using Kramer's affective and cognitive components); 1 all-day workshop for graduates and head nurses on feedback and conflict resolution (using Kramer's behavioral component).

Not specified Six seminars dealing with school to work values conflict (using Kramer's affective and cognitive components); 3 all-day workshops for graduates and head nurses on feedback and conflict resolution (Kramer's behavioral component); preceptors used.

6 months Weekly all-day classes (clinical pathology, nursing interventions, technical skills, primary nursing, leadership, assertiveness, communication skills) and seminars (role transition, conflict management); preceptors used. 
Mims (1984)

Rufo (1984)

Weiss (1984)

Craver \& Sullivan

(1985)

Kasprisin \& Young

(1985)

Marchette \& Merker (1985)

Aldrich (1988)

Ressler, Kruger

\& Herb (1991)
9 months

12 months

2-4 months

6 months

6 months

4 months

2 months

6 months
2 weeks of all-day classes (technical skills, complex care entities); followed by weekly all-day classes on anatomy, physiology, pathophysiology, diagnostic work-ups, assessment techniques, medical management, nursing care management; clinical unit rotations.

All-day educational/feedback sessions; clinical unit rotations; preceptors used.

Classes and seminars: adjustment (psychosocial adaption to role); clinical competency (technical skills and judgment ability); pragmatics of the hospital system (support services, group membership, hospital organization).

Classes (160 hours): pharmacology, fluids and electrolytes, leadership, Bicultural Training; clinical unit rotation; preceptors used.

Four weekly all-day classes on clinical skills; weekly role transitions sessions over 6 months; preceptors used.

Comprehensive, individualized leaming experiences; preceptors used.

Intensive didactic instruction; clinical unit rotation; preceptors used.

Classes and self-leaming packages: pathophysiology, clinical techniques and procedures, physical assessment, pharmacology, organization and priorities, values clarification, stress management, communication skills; clinical unit rotation; preceptors used. 
5) program content - eight included clinical classroom instruction plus role adjustment seminars, two provided clinical classroom instruction only, three used role adjustment seminars only, and one offered a combination of classes with self-leaming packages on clinical topics.

The outcomes reported in the literature concerning these transition programs are reviewed according to the dependent variables in this study, that is: clinical competency, role adjustment, job satisfaction, length of stay and turnover.

Clinical competency. Several studies reported positive findings with regard to the effect of a transition program on neophytes' clinical competency. Using a longitudinal design with both randomly and conveniently selected test subjects and their matched controls, Weiss \& Ramsey (1977) reported statistically significant differences between interns $(n=16)$ and non-interns $(n=16)$ regarding knowledge of medications, use of equipment, effective functioning on evening and night shifts, decision making and priority setting, and work organization. Interns not only perceived themselves as more clinically proficient than controls, but also were rated by their head nurses as more competent than controls in acquiring, performing, and getting feedback on technical skills.

In a different study by Weiss (1984), eight hospitals that employed substantial numbers of new graduates elected to participate in a study using the same protocols employed in the Weiss \& Ramsey (1977) study. All new graduate nurses within these hospitals were selected as subjects. Nurses graduating in the first year of the study were the control group $(n=58)$, while nurses graduating the second year formed the 
experimental group $(n=63)$. The experimental group, or interns, were tested before and after participation in the program and their scores were compared to the non-interns who had had a traditional orientation in the preceding year. Results of $t$-tests for independent samples showed a significant increase in pretest-posttest mean scores on clinical skills competency for both the experimental and control groups; although, despite interns' higher mean score, posttest comparisons between the interns' $(M=73.2)$ and non-interns' $(M=70.6)$ were not significantly different. However, with regard to skills discrepancy, the experimental group demonstrated a significantly greater decrease $\left(t_{1}: M=87.1\right.$, $\mathrm{t}_{2}: M=16.0$ ) in their assessment of their conflict between desired and actual level of clinical skill than did the control group $\left(\mathrm{t}_{1}: M=81.7, \mathrm{t}_{2}: M=11.1 ; t=1.92, p<.05\right)$. According to the author, the interns' negative posttest score indicates they are exceeding their own expectations; that is, their perception of their actual skill level with regard to technical expertise, clinical judgment, and work performance is higher than their expectations of how competent a nurse should be.

Ressler, Kruger \& Herb (1991) compared critical care interns' scores on the Basic Knowledge Assessment Tool (BKAT) to those of experienced nurses who were also newly hired in the study unit. Data was collected at four time points: once upon hire, and three times after the experimental group $(n=24)$ had completed the internship program and the control group $(n=60)$ had completed the traditional orientation. Both groups demonstrated consistent increases in test scores across all time points, with the interns' mean scores the highest at each interval. Results of ANOVAs at $t_{2}$ and six months later at $t_{3}$ showed the interns to have significantly higher mean test scores on the $B K A T$ than 
the control group $(p<.01$ and $p<.05$ respectively), which included nurses with prior critical care experience. In addition to scores on the BKAT, the groups' clinical performance was also compared at $t_{3}$ using job evaluations. Results of ANOVAs revealed no significant differences between the groups' mean scores, with critical care experienced nurses in the control group possessing the highest mean score, followed by the intern group's mean score, with the medical-surgical experienced nurses in the control group having the lowest mean score. One year later at $t_{4}$, there were no statistically significant differences in mean test scores between the experimental and control groups. Frequency and types of medication errors for the two groups were also tabulated at $t_{3}$ and at $t_{4}$. Although the data reported on this measure is incomplete, the authors state that interns demonstrated a higher frequency of medication, IV, and transcription errors at both time points than did the control group nurses.

Positive findings regarding the effect of internship programs are also reported in two other studies. Mims (1984) relates that in comparison to new graduates in the control group $(n=26)$, nurses who participated in a critical care internship program $(n=45)$ perceived themselves as being more technically competent and demonstrated higher test scores measuring clinical knowledge. Kasprisin \& Young (1985) report that 59 interns were perceived by both head nurses and preceptors as able to perform entry level tasks as well or better than the 46 new graduates hired in the preceding year who did not participate in an internship program. It should be noted however, that neither of these studies provided data from which these conclusions are drawn nor exhibit rigor in their research designs. 
In contrast to these positive findings concerning transition programs and clinical competency, several studies report either no significant differences between interns and non-interns or instances where non-interns scored higher than interns. Using a quasiexperimental, pretest-posttest design, Gibbons \& Lewison (1980) reported preliminary findings from a three year research project. Although they report no significant differences between the experimental group $(n=7)$ and the matched control group $(n=7)$ on the Slater Nursing Competencies Rating Scale, it should be noted that the sample was small and t-tests were based on post-internship test scores only. Similarly, Marchette \& Merker (1985) found no significant differences between 13 interns and 13 non-interns in their pretests, adjusted posttests, or pretest to posttest scores concerning self-evaluation of clinical performance. Note, however, that indepth information pertaining to study design and sampling are not provided by these authors.

Studies by May, Minehan \& Deluty (1981) and Craver \& Sullivan (1985), using non-equivalent control group, posttest only designs, and having similarly small convenience sample sizes of 41 and 23 respectively, also found no significant differences between interns and non-interns on overall clinical performance evaluations. However, according to May et al. (1981), non-interns $(M=47.94)$ actually had higher mean scores than the intern group $(M=41.19)$ on the performance subscales measuring clinical knowledge and technical skills $(p<.01)$.

Finally, an investigation of an internship program by Dear, Celentano, Weisman \& Keen (1982), employing a panel time series design, was conducted over three years with data reported at the end of the project. New graduates who selected to participate 
in the internship program constituted the experimental group $(n=28)$ were matched with new graduates receiving a traditional orientation $(n=27)$. These authors reported no significant differences between the two groups on the Slater Nursing Competencies Rating Scale total score, as well as on each subscale of the instrument. In addition, there were no differences between interns and non-interns on clinical performance evaluations conducted by head nurses.

Role adjustment. Four of the studies discussed above regarding the effect of nurse transition programs on clinical competency also reported on changes in neophyte nurses' role adjustment. Weiss \& Ramsey (1977) reported that interns showed a larger increase than control nurses in both service role and professional role conception, indicating a greater alignment to patient care values and a greater identification with the profession of nursing. However, statistically significant increases in professional role conception occurred only among the BSN interns. In addition, whereas the control group demonstrated a decrease in their sense of power and ability to make change in their working environment, such decline did not occur among the intern group. According to the authors, these findings indicate the possibility that internship programs may not only enhance role functioning among some neophytes but also maintain a stable level among others.

In Weiss' (1984) study, comparisons between interns and non-interns regarding role adjustment were analyzed on four subscales. On subscales measuring service role and professional role conception, there were no significant differences between the two groups. On the bureaucratic role conception, however, the experimental group's mean 
score was significantly higher than the control group's (17.0 versus $15.7 ; t=2.38$, $p<.01)$. The author interpreted this finding as suggestive of the internship program's ability to foster new graduates' alignment with rules, regulations, and procedures governing job expectations. In other words, interns are more successful than non-interns at integrating their new work world roles. The fourth subscale concerning role adjustment dealt with role deprivation. On this measure, interns demonstrated a significant decrease in their mean deprivation score from pretest to posttest $\left(\mathrm{t}_{1}: M=21.8\right.$, $\left.\mathrm{t}_{2}: M=19.4 ; t=2.01, p<.05\right)$, while non-interns showed an increase $\left(\mathrm{t}_{1}: M=20.9\right.$, $\mathrm{t}_{2}: M=22.3$ ), although this increase was not statistically significant. In addition, the interns' lower mean score at posttest was not significantly different than the non-interns' mean score $(19.4$ versus $22.3 ; t=1.13$, n.s. $)$.

Gibbons \& Lewison (1980) and Dear et al. (1982) both report on role adjustment outcomes using Rizzo's Role Conflict and Ambiguity Scale. In each study, the experimental group had higher mean scores than the control group, indicating higher levels of role conflict and role ambiguity among interns, but also in each study these findings were statistically non-significant. In addition, Gibbons \& Lewison (1980) report that controls also had a significantly higher mean score on a role transition questionnaire, an instrument developed by the authors which purports to measure subjects' perceptions of their role adaption $(31.50$ versus $26.81, p<.01)$.

Findings regarding the effect of the transition programs on neophytes' role adjustment in studies not also investigating clinical competency were reported by Aldrich (1988). In this study concerning an internship program for nurses employed on 
neuroscience hospital units, a quasi-experimental, pretest-posttest design was used with 32 subjects in the experimental group participating in the internship program and 21 randomly selected subjects in the control group completing the traditional orientation. Within-groups and between-groups comparisons prior to and after internship and orientation programs revealed no significant differences between interns and non-interns. The author concluded that, as in the study by Dear et al. and also argued by those authors, effects of an internship program upon role adjustment of neophyte nurses may not emerge for several years. Thus, study designs which rely on collecting data at intervals based on months may be too limiting for any major change in role conception to occur, regardless of the type of orientation the new graduate receives; and therefore, investigations based on long-term analyses may be more helpful in detecting changes in role conception.

Job satisfaction. Changes in job satisfaction related to neophyte ransition programs are reported in the literature by only a handful of authors. Weiss \& Ramsey (1977) state that prior to the internship, interns as a group were significantly more dissatisfied than non-interns with regard to opportunities to participate in determination of methods, procedures, and setting of goals and to have authority within the hospital. However, post-program data revealed that interns increased in this level of job satisfaction to equal that of the control group. In addition, the control group demonstrated increased dissatisfaction in their assessment of the fairness of working conditions, job security, and financial rewards. Such declines did not occur for the 
experimental group, where job satisfaction increased in relation to both working conditions and the authority and power to share in decisions and goal setting.

As in the Weiss \& Ramsey (1977) study, Weiss (1984) collected data on this dependent variable also using Munson's Job Satisfaction Index, and reporting somewhat similar results. In this study, interns again demonstrated statistically significant increases in job satisfaction from pretest to posttest: on the status of available opportunities subscale, mean scores rose from 11.8 to $13.4(t=1.97, p<.05)$; and on the adequacy of existing opportunities subscale mean scores increased from 24.6 to $26.0(t=2.49$, $p<.01)$. For non-interns, mean scores also increased from pretest to posttest on both subscales, $12.0-12.6$ and 24.6-25.4 respectively, but these changes were not statistically significant. Unfortunately, no information is provided by the author as to comparisons between interns' and non-interns' posttest mean scores on this instrument.

In contrast to these rather positive findings, Gibbons \& Lewison (1980) and Craver \& Sullivan (1985) found no significant differences between interns and noninterns on measures of job satisfaction at the posttest time point, with both studies utilizing the Job Descriptive Index (JDI). Of note, however, in the Gibbons' \& Lewison's (1980) study, both groups experienced decreases from pretest to posttest in the $J D I$ total mean score as well as decreases on three of the five subscales (i.e, work, promotion, and supervision). On the pay and people subscales, interns' mean scores increased while non-interns' decreased. Of these changes, only the increase in the pay subscale score for the intern group is explained by the authors. According to the investigators, interns received 80 percent of the salary received by the control group until 
after completion of the transition program, at which time both groups received the same salary.

Last, Dear et al. (1982), reported no significant changes in job satisfaction over time for either the experimental or control groups using the JDI, stating that both groups' scores remained quite constant. However, on the final JDI total score, the control group $(M=189.6)$ demonstrated a higher level of overall job satisfaction than the experimental group $(M=164.8, p<.05)$. And although on all five subscales, except pay, the groups did not differ significantly, the non-interns' mean scores were higher than the interns.

Length of stay and turnover. The literature pertaining to the effect of transition programs on new graduates' length of stay is severely limited, yet with regard to turnover it is more consistent in its findings. Only May et al. (1981) reported length of stay data. In their study, the mean length of stay for non-interns was 16.55 months compared to interns' 15.62 months, but t-tests were non-significant. In addition, these authors reported non-significant differences between the two groups with regard to turnover rates (TOR), with non-interns having a TOR of $35 \%$ and the interns a TOR of $43 \%$.

In Weiss' (1984) study, non-interns' TOR after 10 months was $31 \%$, compared to interns' TOR for the same length of time of 7\%. And Kasprisin \& Young (1985) reported a TOR among the non-intern group to be $23.9 \%$ and among the intern group 11.9\%. In both studies, the statistical significance of these differences in TOR is not discussed. 
According to Craver \& Sullivan (1985), one year after completion of the internship program, $17 \%$ of the non-interns had resigned in contrast with $3 \%$ of the interns. These authors state that this difference is statistically significant using a Chi square analysis $(p<.022)$, but, given the study's small sample size $(n=29)$, this statistic should be accepted with caution since there is evidence of an insufficient number of subjects per cell for this test.

Studies not previously discussed in this review of the literature on transition programs but which also speak to this outcome variable were related by Holloran, Mishkin \& Hanson (1980), Hollefreund, Mooney, Moore \& Jersan (1981) and Rufo (1984). It should be noted that in each case, the authors provide insufficient information by which to assess the rigor of their study design nor the validity and reliability of their findings. In addition, no information is provided with regard to the statistical significance of the differences between groups' tumover rate. Holloran et al. (1980) report that in the year prior to implementation of their Bicultural Training Program, the TOR for new graduate nurses after 15 months was 41 percent. After implementation of the program, the TOR for all new graduates after 15 months had decreased to $31 \%$. Of this 31 percent, $28 \%$ had not participated in the program versus $3 \%$ who had. Hollefreund et al. (1981) recount that in the two years prior to implementation of a Reality Shock Program, 36\% and $35 \%$ of new graduates resigned within one year. In the succeeding two years, after all new graduates hired attended the mandatory program as part of their orientation, the TOR for new graduates within one year of employment decreased to $13 \%$ and $22 \%$. Finally, Rufo (1984) reports that in comparison to nurses 
who completed traditional orientations and whose TOR was $52.1 \%$ after four years, nurses who had participated in the internship program manifested a 38.6\% TOR for the same time period.

\section{Organizational Theory Perspectives on Employee Values}

Since the time that Kramer and her colleagues initially expressed concern about the professional-bureaucratic role conflicts in nursing, attention to employee values has become a prevalent topic in the organizational literature. For the purposes of this study, the organizational literature on employee values is reviewed from the perspectives of culture (in terms of conflict and excellence) and structure.

Organizational culture. A growing body of management literature deals with corporate culture and cultural values and their relationship to organizational productivity and individuals' work experience. Deal \& Kennedy (1982) claim that strong corporate cultures are the new 'old rule' for business success, and that a strong culture has almost always been the driving force behind continuing success in American business. According to these authors, the strong culture companies that they investigated all had rich and complex systems of values that were shared by the employees.

Posner et al. (1985) investigated the relationship between personal and organizational values among 1,498 managers representing differing levels of management in varying sizes of organizations. The questionnaires used in this study focused on the impact (low, moderate, high) of shared values on the perceptions of personal and organizational vitality. The authors report that their findings demonstrated that shared values were related to: feelings of personal/career success; organizational commitment 
and employee retention; awareness and self-confidence in understanding the values of others and the organization; ethical behavior; feelings of job and personal stress; the importance level to the individual of the organization's goals; and, the importance of organizational stakeholders. The authors concluded that congruence between the values of an organization and its employees, as well as the strength of the relationship, affect both the quality and character of employee commitment and the direction of energy and effort on behalf of the organization.

The role of values in organizations is also central to DePree's (1989) writing. Although his focus is primarily on the concept of leadership and the role of leaders, he clearly articulates a vision for successful organizations that is built upon the relationships of the individuals within them. In describing the art of leadership as "liberating people to do what is required of them in the most effective and humane way possible" (p. 1), he argues for the establishment of a covenantal relationship between the organization and the employee that is based on shared values and meanings. In addition, a covenantal relationship demonstrates genuine caring for, support of, and attention to the intrinsic needs and values of employees. Furthermore, such relationships are grounded in real and meaningful participation in and ownership of the creation of work goals and processes. According to DePree (1989), a covenantal relationship is open to influence and enables organizations to tolerate risk and be "hospitable to the unusual person and unusual ideas" (p. 60).

Shockley-Zalabak \& Morley (1989) investigated the relationship between personal and organizational values and its affect on satisfaction, success, and communication in 
a computer company using a grounded-theory approach. In-depth interviews were conducted with 183 engineers and managers in the company in order to identify sets of employee values and organizational thematic rules (values, beliefs, and assumptions). These sets were then factor analyzed and correlated with multiple variables. The study's findings indicated that organizational rules, when closely aligned with employees' personal values, positively predict the individual's satisfaction and estimation of the quality and success of the organization. In addition, close alignment between organizational rules and personal values was predictive of satisfaction with personal message sending activities and low uncertainty in message receiving. Based on these findings the authors conclude that organizations which broadly involve employees, not just managers, in the ongoing process of value development and articulation are more likely to identify and describe values which correspond to the values of diverse organizational members. Additionally, this diverse involvement at the critical level of values, beliefs, and assumptions is likely to stimulate improved perceptions of overall communication and general expectations about the organization's quality and success.

The alignment and merging of individual and organizational values are also cornerstones to Senge's (1990) notion of a successful organization. According to this author, the innovative, leaming organization is led by the shared vision of its members. Shared vision, as an overarching goal, compels new ways of thinking and acting within organizations, thus it fosters risk taking and experimentation. The source of an organization's shared vision is the personal visions of its members, which in turn arise from individuals' values, concerns, and aspirations. Critical, however, to this process 
is that organizations must have a climate and culture which encourages and enables individuals' to identify and express their personal values and vision. Senge (1990) also notes that an organization's vision, or mission, is not pronounced by management; but instead, is a by-product of interactions of individual visions. As these shared values and visions bubble up from people interacting at many levels within the organization, members experience a sense of common identity and purpose. This, in turn, leads not just to commitment to the vision but more importantly commitment to the organization. Members feel a sense of ownership of both part and whole. According to Senge (1990), the alignment of organizational and workers' values, as well as employees' freedom to function in harmony with their values, is imperative to creating effective work units and achieving organizational goals.

Conflicting values, roles, and expectations as the fundamental sources of strain for the professional entering the work setting are the basic premise of Joseph Raelin's book The Clash of Cultures: Managers Managing Professionals (1991). According to this author, who includes nurses in his discussion, an inherent conflict between managers and professionals in organizations results from a clash between their respective cultures, that is the corporate culture, which captures the commitment of managers, and the professional culture, which socializes professionals.

Managers' education and socialization emphasize interdisciplinary and practical approaches to problem solving. The corporate culture, which strongly influences their socialization, defines the managerial role essentially as articulating the goals of the organization, devising procedures to meet them, and directing others with respect to these 
predetermined means and methods. According to Raelin (1991), this culture is created and driven by a basic set of values that reflect management's continued adherence to the classic principles of bureaucracy: hierarchy, respect for authority, corporate efficiency, teamwork, and career ethic.

Professionals, on the other hand, who work in organizations as salaried employees, are socialized through their disciplines and culture to carry out their technical responsibilities as members of a professional group. The professional group is their reference point with regard to definition of work processes, standards of practice, informal feedback, and evaluation. Thus, their educational background and resulting socialization typically cause them to experience difficulty, especially early in their careers, in conforming to the direction of management due to the perceived loss of professional identity and autonomy. Furthermore, the professional culture is created and sustained by a basic set of values which counters that held by management. The professional value set is composed of a commitment to: (genuine) participation, defiance of authority, corporate effectiveness and social justice, individual initiative, and quality of life ethic.

Thus, the crux of the conflict between professionals and managers lies in their respective, and fundamental, differences in these socialized values, organizational role functioning, and group loyalties. Raelin (1991) also notes that for many novice professionals, their ability to cope with this conflict is seriously deficient and that resolution of the conflict is frequently found through premature job switching or disenchantment with, and in some cases even leaving, the profession. 
A more constructive and productive solution for both parties offered by Raelin (1991) would consist of a collaborative reconceptualization of the specification of both ends and means. Respecification of ends would involve management's clear articulation of organizational values and goals which are usually underspecified. Communicating this vision would give professionals an understanding of the end result required of them. In addition, management must also eliminate their overspecification of means, thereby reducing their control and supervision of professionals' operational decisions. Such freedom would allow professionals the desired, and socialized, autonomy to practice in accordance with their values, knowledge, and skills.

Furthermore, the realization of this solution, according to Raelin (1991), is contingent on organizations and managers overcoming their impersonalness and adopting humanistic values. Drawing upon the work of Douglas McGregor and Chris Argyris, the author describes humanistic values as being guided by four principles: consensus, trust, egalitarianism, and satisfaction of human needs. Consensus deals with an approach to decision making that is bottom-up and involves all individuals directly affected. Trust, a by-product of consensus decision making, allows individuals to take initiative without others fearing that hidden or illicit motives guide their efforts. Egalitarianism concerns the breaking down of formal roles between individuals in the organization and the deemphasis of rank and position. Satisfaction of human needs pertains to management's responsibility for providing opportunities for employees to grow and mature both personally and professionally. 
Support for Raelin's (1991) description of the conflicting value sets of managers and professionals can be found in a study by Grey \& Gelfond (1990). In a nationwide survey of middle managers, professionals, clerical, and hourly employees these authors found that the number one value among all groups was pay and benefits. The next most important work values held by both managers and professionals were job challenge and opportunities for advancement. After that, however, the value set differences between managers and professionals emerged in the following manner: (in descending order of importance) managers valued authority, supervision, and accomplishment; while professionals valued new skills, respect, and supervision. In addition, only about $50 \%$ of the professionals surveyed felt positive about their chances to develop new skills and talents and to experience a real sense of professional accomplishment.

Organizational Structure. Complementing the literature on values from a cultural perspective, another growing body of organizational literature deals with the nature of bureaucratically structured organizations as both source and sustenance for values conflict and poor organizational performance. Although bureaucratic structures can be useful in some organizations because of their efficiency in the performance of certain tasks and ability to reinforce and sustain particular patterns of power and control, their mechanistic orientation has severe limitations, especially in today's environment. Morgan (1986) identified four major limitations of the bureaucratic model: 1) it creates organizational forms that adapt poorly to changing circumstances; 2) it often results in mindless and unquestioned rules and procedures; 3) it encourages the development of subgoals and sets of interests that undermine the organization's primary goals; and 4) it 
tends to dehumanize employees and limit rather than mobilize the development of human capacities.

Numerous other publications have described the deficiencies of the bureaucratic model, the evidence of its absence in successful organizations today, and its anticipated further demise in successful and entrepreneurial organizations of the future (Kanter, 1983, 1989; Kofman \& Senge, 1993; Peters \& Waterman, 1982; Senge, 1990). Although this literature is primarily referenced with regard to organizational performance and innovation, the negative consequences of bureaucratically structured organizations on employees and their work experiences is also a pervasive theme. It is generally agreed that attention to employee values from an organizational perspective cannot be fully dealt with without a parallel concem with organizational structure.

Despite the progress described in much of the literature regarding organizations' advances in becoming more humanistic and responsive to employee values, some writers support Raelin's (1991) contention that the basic principles of bureaucracy continue to flourish in organizations today. In fact, a small contingent of authors argues that the bureaucratic model actually continues to dominate current organizations. The contention is that the bureaucratic tradition, with its reliance on formal structure, authority, efficiency, rationality, and impersonalness, remains deeply and thoroughly ingrained in current conceptions of organization and people in organizations (Clark, 1985; Clark \& Meloy, 1988; Morgan, 1986; Weick, 1979, 1985). Morgan (1986) states, "for many people it is almost second nature to organize by setting up a structure of clearly defined 
activities linked by clear lines of command, communication, coordination, and control" (p. 33).

According to Clark (1985), the basic assumptions and principles of the bureaucratic model (e.g., efficiency, calculability, substantive rationality, technical competence, formalistic impersonality, universality) continue to be viewed as valid and thus go unchallenged. As Clark (1985) states, the traditional bureaucratic structure "has been built upon, rationalized, adjusted, twisted, and modified for the past forty years, but its essential assumptions still govern the popular conception of organizations and administrators, the training programs for administrators in our colleges and universities, the research that is undertaken in the field, the development activities that produce our most usable and used technologies, and the way we talk about our work places" (p. 51). Hence, many of the alternative organizational models advocated in the current literature continue to reflect this "root" paradigm and thus fail to achieve meaningful and lasting solutions.

Real and successful alternatives to the bureaucratic model, on the other hand, require a transformation of thinking about people and organizations as well as fundamental changes in the structuring of organizations (Clark, 1985; Morgan, 1986; Schaef \& Wilson, 1988; Weick, 1979, 1985). For instance, Kofman \& Senge (1993) describe organizations that are "communities of commitment." Such organizations would be grounded in three principles: a culture based on transcendent human values of love, wonder, humility, and compassion; a set of practices for generative conversation and coordinated action; and, a capacity to see and work with the complex whole. 
Clark \& Meloy (1988) propose the creation of organizational structures that arise from the concepts of democracy and McGregor's Theory X and Theory Y. In such structures, our values and assumptions about people would be aligned (or conjoined) with our values and assumptions about organizational structure. Consequently, instead of having organizations that espouse a Theory $\mathrm{Y}$ view of people while operating with Theory X-type structures and processes; innovative and successful organizations would create $\mathrm{Y}-\mathrm{Y}$ structures. The $\mathrm{Y}-\mathrm{Y}$ organization would be structured "with the individual as its building block, exhibiting a total regard for persons. [It] would trade off control for empowerment, domination for freedom, and authority for consent. [It] would choose its leaders, concern itself with the self-actualization of all its members, share the power tools of the organization, de-emphasize hierarchical relationships, and create opportunities for self-fulfilling jobs" (Clark \& Meloy, 1988, p. 278).

\section{Nursing Literature and Research on Employee Values}

The influence of the organizational literature on corporate culture and cultural values and their relationship to organizational productivity and individuals' work experience is becoming evident in the nursing literature. In 1982, the American Nurses' Association (ANA) sponsored the original magnet hospital study (McClure, Poulin, Sovie \& Wandelt, 1982) which resulted in the designation of 41 hospitals across the nation as magnet hospitals. The criteria used for the selection these 41 hospitals were that they had been particularly successful in attracting and retaining professional nursing staff, and had reputations as being good places to work and as giving quality nursing care. In this original study, as well as in follow-up studies involving a subset of the initial sample, it 
was found that these hospitals all possessed the attributes of a culture of excellence: experimentation, core value formulation, and recognition of the competence and autonomy of the individual (Kramer, 1990a, 1990b; Kramer \& Schmalenberg, 1988a, 1988b; Kramer \& Schmalenberg, 1991a, 1991b). In particular, the magnet hospitals were renowned for their use of non-bureaucratic organizational stuctures, such as decentralization, shared governance, and career ladders. Moreover, these studies revealed significant organizational attention to the professional and personal values of the nursing staff and a sense of shared values with nursing and hospital administration. Additionally, the presence of these attributes of excellence correlated with high job satisfaction and high self-esteem among the nurses.

The influence of the organizational literature is also evident in nurses' concern with the necessity of attending to organizational culture, as well as the implications and anticipated outcomes associated with doing so (Caroselli, 1992; Coeling \& Simms, 1993a, 1993b; Curran \& Miller, 1990; del Bueno \& Vincent, 1986; Nyberg, 1991; Sovie, 1993; Thomas, Ward, Chorba, \& Kumiega, 1990). In addition, a few nursing research studies are beginning to appear which examine organizational culture and its relationship to a variety of work setting issues (Coeling \& Wilcox, 1988; Kramer \& Hafner, 1989; McDaniel \& Stumpf, 1993). However, whereas this literature deals with staff nurses in general, only a handful of studies, apart from those previously reviewed related to transition programs, have explored the characteristics and consequences of the professional and role-oriented values possessed by new graduate staff nurses. 
In a longitudinal study of 135 new graduate nurses in a large medical center, data were collected during the first month of employment with regard to their professional and bureaucratic value orientations (Speedling, Ahmadi, \& Kuhn-Weissman, 1981). In one set of questions, respondents were asked to rate the importance of several items which reflected either a task-value or a patient-value within the professional orientation, and to select the one item which they thought was most important: $49 \%$ chose areas which were task oriented (extensive knowledge of current nursing practice, and current knowledge of relevant therapy); and $44 \%$ chose areas which were patient oriented (ability to establish rapport with patients, and skill in dealing with social and psychological problems of patients). Other areas were insignificant, including working with doctors and supervisors, which ranked last and next to last, respectively.

In another set of questions, a professional value orientation was measured on a Likert-type scale using dimensions that deal primarily with loyalty to the nursing profession: involvement in professional organizations, scientific knowledge as the basis for practice, exercise of professional judgment in decision-making. The values inherent in the bureaucratic role conception, also measured on a Likert-type scale, were loyalty to the employing institution and those in authority, and following administrative rules and routines. Dividing the sample at the mean resulted in the following distribution:

$\begin{array}{ll}\text { Low Bureaucratic(LB)-Low Professional(LP) } & 26.6 \% \\ \text { High Bureaucratic(HB)-High Professional(HP) } & 24.9 \% \\ \text { Low Bureaucratic-High Professional } & 23.7 \% \\ \text { High Bureaucratic-Low Professional } & 24.9 \%\end{array}$

Data from these two sets of questions were also correlated with each other with the following findings: nurses in the categories LB-HP and HB-LP were more frequently 
found in patient-value categories, while LB-LP and HB-HP nurses were more frequently in task-value categories. In addition, new graduates who were high both in bureaucratic and professional orientations were higher on a scale measuring powerlessness.

Further examination of the data from this study was reported by the same authors in a subsequent publication (Ahmadi, Speedling, \& Kuhn-Weissman, 1987). New graduate nurses who had completed questionnaires during the first month of employment regarding their professional and bureaucratic value orientations, importance of job factors, satisfaction, and powerlessness were asked to complete the questionnaires again after 12 months. Between time of hire and a year later, mean bureaucratic (ideal) and professional (ideal) role conceptions, professional role discrepancy (ideal-real) and job satisfaction remained essentially unchanged. During this interval, however, mean bureaucratic actual situation (real), bureaucratic role discrepancy (ideal-real), and powerlessness increased, while professional actual situation (real) and importance of job factors decreased. In addition, bureaucratic role conception and bureaucratic actual situation were both negatively correlated with length of stay at termination; and, professional role conception was negatively correlated with both powerlessness and job satisfaction. The authors conclude that, although some of their findings do not confirm those reported by Kramer (1970), the results support the idea that bureaucratic and professional allegiances may operate in opposition to each other; and, that the newly graduated nurse, faced with discrepancies between school-taught values and workplace values, may develop a sense of powerlessness and job dissatisfaction. 
In a study by Green (1988), the relationships between role models and role perceptions were examined. The sample consisted on 25 senior nursing students in a baccalaureate nursing program who were administered questionnaires one month prior to graduation and three months after beginning hospital employment. Results of the study indicated that a majority of faculty role models of new graduates were replaced by work-related role models and that the most important role model characteristics were clinical experience and clinical performance. Role conception was measured in two ways: the first according to respondents perceptions of what is ideal in nursing, and the second according to their perceptions of what is really observed in nursing. From the first to the second time points: respondents demonstrated a $36 \%(p<.05)$ decrease in their ideal professional role conception and a $16 \%$ (n.s.) increase in their ideal bureaucratic role conception; and, a $16 \%$ (n.s.) decrease in their observed professional role orientation and a $28 \%(p<.05)$ increase in their bureaucratic role orientation.

Gardner (1992) examined the relationship between conflict and retention among 166 new graduates employed in a large midwestern hospital. Data were collected after six months and 12 months of employment. Subjects' mean scores for professional role conflict increased from 3.65 to 3.82 , however this change was not statistically significant, and there were also no significant differences in role conflict scores for nurses who stayed and those who terminated employment. Tests of correlation revealed no significant association between professional role conflict and turnover; however, job satisfaction was significantly negatively correlated with role conflict at both time points. 


\section{$\underline{\text { Summary }}$}

A review of the literature regarding the effect of transition programs on new graduate nurses' clinical competency, role adjustment, job satisfaction, and length of stay yields mixed and inconclusive findings. No clearly discernable, objective trend emerges as to either the outcomes or benefits of these programs with respect to these four variables. In contrast, the findings with regard to turnover are much more consistent and point in a positive direction. However, it is unknown to what extent these indications of decreased new graduate turnover are a result of the transition programs per se versus other organizational factors and events in the study hospitals that may influence a decline in nurses' turnover, and that are not measured, controlled for, or addressed, in these investigations. Furthermore, the apparent decrease in turnover as cited in these studies may reflect the overall national trend of reduced hospital staff nurse turnover which many hospital and nursing administrators have felt is due to the increasing instability in job markets and the economic downtum seen throughout the country beginning in the last decade. In other words, in light of shrinking employment opportunities and organizational downsizing occurring in many professions and industries, nursing's relatively stable job market and employment security may serve to increase nurses' job retention as they become a family's primary provider.

However, as evidenced in this review, nursing research in this area has attempted to extend the work originally introduced by Kramer, Schmalenberg, and Benner. In addition, this review reveals an important and positive feature in nursing service research. That is, although most transition programs were implemented with a stated 
intent to meet an institutional need, all the programs have essentially the same objective-to provide new graduate nurses with the opportunity to increase their clinical knowledge, skills, and self-confidence while making a smooth transition to the staff nurse role. Thus, hospitals have initiated these programs to meet the needs of new graduates as well as their own needs for cost containment and for the provision of patient care by competent, confident, and satisfied personnel.

Yet, frequently this body of research also exhibits serious weaknesses with regard to sampling techniques, generalizability, sample size, selection of instruments with sound psychometric properties, and data analyses. In addition to the inadequate rigor in overall study design, the significant variability in the transition programs' structure, process, and content from one study to another (see Table 1) hinders the ability to draw reliable conclusions from their findings. Furthermore, information provided by the authors is often incomplete and replete with opinions and suppositions as to the outcomes of these programs. It should be noted, however, that as is common in much of nursing service research, the notion of conducting a research study is often an after-thought, and thus frequently it is too late to introduce appropriate and sound study protocols.

The pragmatic approach to problem solving displayed in this body of nursing research has brought about not only a proliferation of short-term, institution-specific studies but also a serious lack of systematic and objective evaluation as to the long-term outcomes of transition programs. The major strength of this study is found in its large sample size $(\mathrm{n}=890)$ and its utilization of long-term data $(1983-1992)$. Thus the study has potential not only to detect significant changes in subjects regarding the variables 
under study but also to disclose meaningful and important information as to the benefits of neophyte nurse transition programs.

With regard to employee values, the fact that this topic is currently such a prevalent theme in the organizational literature and an increasingly popular issue in the nursing literature demonstrates both the importance of the theoretical work advanced by Kramer and her colleagues in the 1970's and its continued relevance today. In addition, the magnet hospitals studies have provided strong support of the notion that organizational structuring is a pivotal component to nurses' professional practice and job satisfaction. However, in general, review of the nursing literature reveals more opinion than empirical study of nurses' professional values and their relationship to employment issues. Furthermore, the research pertaining to the characteristics and consequences of the professional and role-oriented values possessed by new graduate staff nurses is quite limited. Yet, there is continued evidence that new graduate nurses experience changes in their value orientation over time, especially during the first year of employment, and that these changes may affect nurses' satisfaction with their work experience and employment tenure. This study sought to expand the knowledge base on new graduate nurses' professional and role-oriented values by identifying the values neophyte nurses possessed upon initial employment, and determining whether these values changed over time and affected new graduates' length of stay. 


\section{Chapter III}

\section{METHODOLOGY}

\section{Design}

This study addressed the following questions: 1) What is the effect of a nurse transition program on neophyte staff nurses' clinical competency, role adjustment, job satisfaction, length of stay and turnover?; and 2) What are the nature and discriminating characteristics of neophyte nurses' professional and role-oriented values? The purpose of this study was to assess whether, and to what degree, new graduate nurses' clinical competency, role adjustment, job satisfaction, and length of stay increase, and their rate of turnover decreases, as a result of their participation in a transition program. In addition, this study sought to determine if, among new graduates participating in the transition program, there are differences with regard to clinical competency, role adjustment, and job satisfaction between those who stayed beyond 15 months after hire (stayers) and those who terminated employment within 15 months (leavers). Relatedly, this study sought to identify the professional and role-oriented values new nurses possessed upon initial employment, to determine whether these values changed over time, and to discern whether differing values affected the nurses' length of stay.

Specifically, this study was guided by the following research questions:

1) Do neophyte nurses participating in the transition program demonstrate a significant increase over time in their clinical competency, professional role adjustment, and job satisfaction? 
2) Is the length of stay of neophyte nurses participating in the transition program longer than the length of stay of new graduate nurses employed prior to implementation of the transition program?

3) Is the turnover rate of neophyte nurses participating in the transition program less than the turnover rate of new graduate nurses employed prior to implementation of the transition program?

4) Are there significant differences with regard to clinical competency, role adjustment, and job satisfaction between neophyte nurses whose length of stay exceeds 15 months of (stayers) and neophyte nurses who resign within 15 months of employment (leavers)?

5) What professional and role-oriented values do neophyte nurses possess upon initial employment?

6) Do neophyte nurses' professional and role-oriented values change over time?

7) Are there significant differences with regard to professional and roleoriented values between neophyte nurses whose length of stay exceeds 15 months (stayers) and neophyte nurses who resign within 15 months of employment (leavers)?

To answer these questions, this study used secondary data and employed: 1) a 9x3 nonrandomized repeated measures design, combining one within-subjects factor (time; three levels: intake, and 6 and 12 months later) with one between-subjects factor (panel year; nine levels: 1983, 1984, 1985, 1986, 1987, 1988, 1989, 1990, and 1991); 
2) a $2 \times 3$ nonrandomized repeated measures design, combining one within-subjects factor (time; three levels: intake, and 6 and 12 months later) with one between-subjects factor (length of stay; two levels: stayers and leavers); 3) factor analysis of two of the instruments used in the study; and 4) reiteration of the $9 \times 3$ and a $2 \times 3$ repeated measures designs using the factor scores obtained from the factor analyses.

The secondary data were obtained using a longitudinal, panel study design (Final Report, 1987' Burns \& Grove, 1987; Polit \& Hungler, 1987) in which each panel was composed of new graduate nurses employed annually by the hospital study site beginning in 1983 and ending in 1991. All subjects in each of the nine panels participated in the transition program developed by the hospital and completed the same battery of instruments measuring the outcome variables of clinical competency, role adjustment, and job satisfaction. Data were collected on each of these three variables at three time points: on entry to work $\left(t_{1}\right)$; after six months of employment $\left(t_{2}\right)$; and after 12 months of employment $\left(t_{3}\right)$. Data regarding the outcome variables of length of stay and turnover were collected by ongoing tracking of subjects' dates of employment terminations.

\section{Setting and Sample}

The study site from which this data set was obtained is a 560 bed, tertiary care, regional medical center located in the southeast region of the United States. Permission

${ }^{1}$ This unpublished document, written by transition program staff at the original study site hospital, describes the procedures and findings regarding the transition program from 1983-1985. In the original study, outcome data from 1983-1985 were compared to outcome data collected from a baseline, pre-program implementation group in 1981. With the exception of aggregate length of stay and turnover rate, the data from the contrast group are not available for this secondary analysis. 
to use the data for secondary analysis was obtained from the hospital's president and the director of the department of nursing.

The sample for this study was composed of all new graduate nurses hired as staff nurses by the hospital study site beginning in 1983 and ending in 1991 . Therefore, this convenience sample consisted of 890 subjects who were newly graduated from diploma, associate degree, or baccalaureate degree nursing programs; sought and obtained employment at this medical center; and were hired prior to or recently after taking the national licensing examination. The number of subjects per panel year in the sample ranged from 68 to 155 .

Subjects' consent to inclusion in the study was obtained at the time of initial hiring by the hospital. That is, when new graduate nurses accepted employment at the hospital study site, they agread to participate in the procedures and evaluation activities associated with the transition program and signed a consent form to that effect (see Appendix A).

\section{Description of the Independent Variable}

Due to changes in the neophyte transition program's funding sources, some procedures and activities associated with the independent variable were changed over the ten year period that the data were collected. These changes essentially fell into two time periods: one from 1983 to 1987 and the other from 1988 to 1992.

Program procedures from 1983 to 1987 . During this time period, the procedures associated with the transition program at the hospital study site were modeled after, and designed to replicate, the program activities reported by Weiss \& Ramsey 
(1977) and Weiss (1984). The program focused on three components, or goals, related to facilitating the transition from student to staff nurse: 1) clinical competency - to increase technical and decision making skills on the clinical unit; 2) adjustment -to assist in the psychosocial adaptation to the staff nurse role; and 3) pragmatics of the hospital system - to increase understanding of the hospital environment. Utilizing competencybased centralized and decentralized activities, the transition program addressed these goals in four phases over the course of 12 months.

Phase I: General orientation. During the first week of employment, new graduates attended centralized orientation sessions which covered the hospital and department of nursing in general. In addition, subjects attended the seminar "Getting into the Group" which focused on the process and strategies of establishing oneself as a group member. In week two, decentralized unit orientations to the assigned patient care unit were conducted.

Phase II: Assessment of clinical skills and contracting. During this phase, beginning in week three and lasting until the end of six months, each new graduate conducted a self-evaluation of her clinical skills with regard to patient and family assessment, nursing interventions, medication administration, documentation, risk management, and patient support. Appendix B lists the statements for each clinical competency. For each behaviorally-anchored competency, the participant evaluated whether s/he was ready to demonstrate the competency in the clinical setting or needed to contract for leaming activities in order to develop the competency. Demonstrations of competencies and/or contracting for leaming activities was then performed with the 
head nurse and the clinical instructor assigned to the area. Contracts included identification of leaming need, negotiation for experiences to meet those needs, date for completion, and criteria for evaluation. Methods for meeting the identified learning needs included didactic classroom instruction, hands-on skills laboratory activities, reading materials, use of self-paced notebooks and computer-assisted modules, viewing of video tapes, caring for selected patients on the nursing unit, and observations of patient procedures in the hospital's clinical departments and labs.

Phase III: Adjustment and pragmatics of the hospital system. This phase, beginning in the fifth week, focused on assisting the new graduates' adjustment to the hospital environment and the staff nurse role. Seven different seminar sessions were available and participants were scheduled to attend each of the sessions at between the 5th and 21st week of employment. The seven seminars were: "Using Clinical Judgment," "Selecting Priorities," "Coping With The New Role," "Coping With Others," "Working Together," "Understanding The Hospital System," and "Blending Independence And The System." The seminars were led by nurse educators from the hospital and local schools of nursing and included viewing of video tapes, role playing, and group discussions. The competency statement and objectives for each seminar are listed in Appendix B.

Phase IV: Evaluation. At the end of six months, program participants evaluated their progress to date in joint conference with their head nurses and clinical instructors with regard to clinical competencies, role adjustment and hospital pragmatics. At this time, new contracts were developed if the required competencies had yet to be met. At 
the end of twelve months, or one year of employment, an annual performance evaluation was conducted.

Program procedures from 1987 to 1992. Beginning in 1987, some of the transition program's procedures were modified. The program's first goal regarding clinical competency remained the same; and no changes were made in either the data collection instruments or the times at which test batteries were administered. However, the program's formal structure was reduced to three months and its third goal regarding pragmatics of the hospital system was eliminated. These modifications were reflected within the program's phases as well.

Phase I: General orientation. During the first week of employment, new graduates still attended centralized orientation sessions which covered the hospital and department of nursing in general. However, the original seminar was replaced with a new seminar entitled "Thriving and Surviving," which dealt with the student to staff nurse ransition process and strategies for making a successful adjustment.

Phase II: Unit orientation, assessment of clinical skills and contracting. Beginning in week two, participants were oriented to their respective patient care units and began their self-evaluation of clinical skills. The list of competencies, processes for demonstration or contracting, and learning activities did not change. However, the head nurse's role in competency assessment and contracting was now performed by each participant's unit preceptor.

Phase III: Adjustment. The seven seminars were replaced with one day-long workshop which dealt with decision making and adjustment to the staff nurse role. The 
workshop was held during week twelve, which was the last week of the program's formal structure. In addition to the new graduates, these seminars were to be attended by the preceptors. See Appendix B for the workshop's objectives.

Phase IV: Evaluation. At the end of three months, competency evaluations involving participants, preceptors and clinical instructors occurred and contracts were renegotiated as needed. At the end of twelve months, or one year of employment, an annual performance evaluation was conducted.

\section{Dependent Measures}

The data used in this study were obtained from two sources: 1) instruments completed by participants in the transition program; and 2) department of nursing personnel records.

Instruments. Data were collected on the variables of clinical competency, role adjustment, and job satisfaction at three time points: on entry to work ( $\left.\mathrm{t}_{1}\right)$; after six months of employment $\left(t_{2}\right)$; and after 12 months of employment $\left(t_{3}\right)$. The test battery given to subjects at these three time points included five questionnaires. Copies of these instruments are included in Appendix C.

Profile data sheet. This form elicited participant information on 12 force-choice items relative to demographics, education, prior employment status, clinical interest, and career aspirations. It was constructed and evaluated for face validity by staff at the original study site.

Nursing Competency Assessment Scale (NCA). This self-rated instrument was devised by the clinical instructor group at the hospital study site to measure the outcome 
variable of clinical competence. The NCA divides nursing activities into seven categories: assessment, intervention, medication administration, documentation, risk management, patient support, and professionalization. There are 30 items on the scale (two to seven items per subscale) which uses a five-point Likert type rating from low (1) to high (5) level of proficiency. Scores may range from 30 to 150 for the NCA total, with subscales' scores ranging as follows: assessment (7-35); intervention (4-20); medication administration (2-10); documentation (3-15); risk management (3-15); patient support (5-25); and professionalization (6-30). An overall mean score for the NCA is determined, as well as a mean score for each of the seven subscales. This tool was reviewed by an expert panel for face and content validity, and reliability coefficients in the original study were reported to range from .76 to .90 (Final Report, 1987).

Nursing Characteristics Scale (NCS). Developed by William Holzemer at the University of Califormia at San Francisco (Final Report, 1987), this scale addressed the outcome variable of role adjustment. The instrument was designed to assess respondents' degree of idealism, realism, and disillusionment regarding the actual practice of nursing in the work world. The scale requested respondents to rate 14 attributes or characteristics of nursing practice (such as opportunities for advancement, leadership functions, and role status) along a five-point scale of increasing intensity, so that the higher the score, the more positive was the perception. Scores may range from 14 to 70 and a total mean value for the 14 items is reported. A reliability coefficient (Cronbach's alpha) of .81 was reported for the original study (Final Report, 1987). 
Staff Nurse Role Function Survey (RFS). Designed to measure the outcome variable of role adjustment, this questionnaire was developed by staff at the hospital study site using a group of head nurses from multiple representative hospitals as expert panels. The survey contained 21 items representing competencies deemed integral to the staff nurse role, and which, when measured over time, reflected familiarity with work demands and the hospital environment. The scale provided for subjects to self-rate their current level of confidence on each role function item using a five-point continuum from unsure (1) to very confident (5). A total mean score for the 21 items is reported with scores ranging from 21 to 105 . Instrument validity was addressed by expert review and by positive correlations with the Nursing Characteristics Scale. In the original study, reliability coefficients were reported to be .92 , with all items having a Cronbach's alpha over .90 (Final Report, 1987).

Job Satisfaction Index (JSI). This instrument measured the outcome variable of job satisfaction along four dimensions: intrinsic, involvement, interpersonal, and extrinsic. Intrinsic satisfaction included the opportunity to fully use skills and abilities on the job, to do important and worthwhile things, and to realize one's potential (items $1,2,3,4)$. Involvement satisfaction referred to the authority to share in the determination of methods and procedures, setting goals, and directing others on the job (items 9,14 , 15, 16). Interpersonal satisfaction described the opportunity to work closely with likable people, to have problems and difficulties understood by others, and to give help to others (items 10, 11, 12, 13). Extrinsic satisfaction included the fairness of working conditions, job security, and financial rewards (items 5, 6, 7, 8). Responses are on a five-point 
scale from dissatisfied (1) to very satisfied (5). Total scores on the instrument may range from 16 to 80 , with subscale scores ranging from 4 to 20 . An overall mean score for the index is reported, as well as a mean score for each of the four subscales. The scale was adapted from Munson's Job Satisfaction Index (Munson \& Heda, 1974) by staff at the hospital study site. Modifications included the addition of four items and a reduction in the scaling points from seven to five. Use of this modified version of the instrument in the original study reportedly demonstrated an internal consistency (Cronbach's alpha) coefficient of .90 (Final Report, 1987).

Personnel Records. Data regarding the outcome variables of length of stay and turnover were collected by ongoing tracking and entry of subjects' dates of employment terminations. In order to verify and update this data, department of nursing personnel records were reviewed by staff at the study site and dates of termination were obtained for all subjects who had resigned their positions by November 1992. In addition, subjects with continuing-employment status were also verified during this review of the personnel records.

Based on subjects' dates of employment and dates of termination or continuingemployment status, the outcome variable of length of stay, measured in months and years, was calculated. Turnover rates were determined by dividing the number of subjects who resigned yearly for the first three years by the total number of subjects in the group, then multiplying by 100 to yield a percent rate. 


\section{Data Analysis Procedures}

Data preparation. The secondary data set used in this study, which was written in dBase II, required numerous conditioning procedures before it was ready for analysis. This conditioning process included: cleaning for missing data; changing fields from character to numeric; restructuring from multiple-entry fields to single-entry fields; and some recoding to create total-score fields. With regard to missing data, cases with more than one data time point missing were deleted from the data analyses. In addition, notation was made of: subjects whose date of entry was after November 1991 since those subjects would not have completed a full 12-month transition program; and subjects whose date of entry was after August 1991 since their length of stay with respect to the 15-month cut-off could not be determined and therefore they could not be assigned to the stayers or leavers groups. Upon completion of the conditioning procedures, the data were imported into and analyzed using SPSS/PC+.

Descriptive Statistics. Descriptive statistics on the demographic variables and on all test variables were utilized to provide a description of the sample's demographic characteristics, and for description of the test variables' distributions as part of checking the assumptions for the planned statistical tests. In addition, chi-square analyses were conducted on the demographic variables to determine whether the nine panel groups were drawn from the same population and thus whether the aggregate sample was homogenous with respect to demographic characteristics. These tests were also performed on the groups designated as stayers and leavers to determine if there were demographic differences between the two groups. 
Analysis of Variance. Cross-sectional analyses of variance were performed on data collected at entry $\left(t_{1}\right)$ with respect to the outcome variables of clinical competency, role adjustment, and job satisfaction to determine whether the nine panel groups significantly differ on these measures prior to their participation in the transition program and thus whether the aggregate sample was homogenous with respect to these variables. One-way ANOVAs were used to allow for the inclusion of post hoc tests. The data were also evaluated for the assumptions of the ANOVA test: independence, normality, and homogeneity of variance (Kohler, 1988). In cases where the assumptions are violated, the one-way ANOVAs nonparametric equivalent, the Kruskal-Wallis test, was used.

In order to evaluate whether subjects' scores changed over time (longitudinal analysis for $9 \times 3$ design), between-subjects (panel year) repeated measures analyses of variance (RM-ANOVAs) were conducted on the outcome variables of clinical competency, role adjustment, and job satisfaction with the three time points serving as the within-subjects factor. Post hoc tests were included to determine whether there were significant differences between the nine panels and where the differences, if any, existed. In addition, one-sample RM-ANOVAs were performed for each panel group to provide within-subjects measures on the outcome variables across the three time points. Differences between stayers and leavers on the dependent variables (longitudinal analyses for $2 \times 3$ design) were evaluated using between-subjects (stayers versus leavers) RMANOVAs on the outcome variables of clinical competency, role adjustment, and job satisfaction with the three time points serving as the within-subjects factor. 
RM-ANOVAs are applications of the univariate analysis of variance model and are designed to evaluate differences in means across various time points on a sequence of dependent variables, with the possibility of also examining for differences in means between groups (between-subjects) and the (within-subjects) interaction of time and group (Bray \& Maxwell, 1985; Toothaker, 1991; Winer, 1971). Longitudinal analyses such as RM-ANOVA pose less sample size demands and are associated with greater precision and power (Roberts, 1989; Waltz \& Bausell, 1981; Winer, 1971).

The data were evaluated with regard to the governing statistical assumptions, which include: 1) observations are statistically independent of one another; 2) the dependent variables have a normal distribution; and 3) the $k$ groups present with homogeneity of variances (Stevens, 1992). According to Bray \& Maxwell (1985), ANOVA models are relatively robust to violations of the assumptions in many situations, although they are least robust with regard to the assumption of independence.

Length of stay and turnover rates were calculated for each panel year, for the groups designated as stayers and leavers, and for the aggregate sample. These values were grossly compared to the respective pre-program values. No statistical tests were conducted since the pre-program data was available only in an aggregate, summarized form.

Factor Analysis. In order to identify new graduates' professional and roleoriented values, two separate exploratory factor analyses (FA) were performed using subjects' scores obtained at $\mathrm{t}_{1}$ on the Nursing Characteristics Scale (NCS) and the Role Function Survey (RFS). The goal of factor analysis is to identify a relatively small 
number of factors for the purpose of representing relationships among sets of many interrelated variables. By identifying the not-directly-observable factors based on a set of observable variables, meaningful complexity reduction of a data set can be achieved. In short, factor analysis helps identify underlying constructs on the basis of observed correlations between variables that reflect commonality of constructs. The NCS and the RFS were chosen because the scales' items were assumed to be reflective of the intrinsic parameters that nurses might use to evaluate the nursing profession and their role as a staff nurse. Hence, the grouping of such items into factors may indicate underlying constructs, or values, possessed and shared by these new graduate nurses.

Prior to performing the FAs, the data were evaluated for their ability to generate statistically stable factor structures using the rule of $10: 1$ for the observation-to-variables ratio. In addition, the correlation matrix for each scale was examined to determine the appropriateness of the factor models (Tabachnick \& Fidell, 1989; Norusis 1988). The suitability of each correlation matrix was determined by the Kaiser-Meyer-Olkin (KMO) estimate of sampling adequacy, Bartlett's test of sphericity, and the number of offdiagonal elements greater than .09 in the anti-image correlation matrix (\% AIC).

For conducting both of the FAs, initial factor extraction was done using the principal components method (PCA). This method was chosen because it forms factors which are uncorrelated with each other and because of its ability to explain as much of the total variance in the data as possible with as few factors, or components, as possible (Kleinbaum, Kupper, \& Muller, 1988; Tabachnick \& Fidell, 1989). In addition, because PCA analyzes all the variance in the observed variables, it is recommended in situations 
where an empirical summary of the data set is desired, and when the factors may be used in other analyses, e.g., as dependent variables in RM-ANOVA (Stevens, 1992; Tabachnick \& Fidell, 1989). The criteria of a minimum eigenvalue of 1.0 was also utilized in order to determine the number of factors to be retained. According to Stevens (1992), Kaiser's rule (eigenvalues $\geq 1.00$ ) has been shown to be quite accurate when the sample size is greater than 250 and the mean communality is $\geq .60$. However, because one of the FAs did not possess an adequate mean communality, the Cattell scree test (obtained during initial component extraction without designation of a minimum eigenvalue criteria) was also used for confirming the number of factors appropriate for retention.

The factor solutions were rotated using the varimax approach. Varimax rotation was selected for several reasons. First, as an orthogonal rotation, the original principal components remain uncorrelated with each other, and therefore, consistent with using the PCA extraction method, factor independence is optimized. Second, since the goal of varimax rotation is to simplify factors by maximizing the variance of the loadings within factors and across variables, interpreting a factor is easier because it is obvious which variables correlate with it. And third, varimax tends to reapportion variance among factors so that they become relatively equal in importance, that is variance is taken from the first factors extracted and distributed among the later ones (Tabachnick \& Fidell, 1989). After rotation, the final factors were identified based on the significance of the factor loadings. To accomplish this, the factors were examined for loadings that 
accounted for at least $10 \%$ of the variance of a factor. Accordingly, .32 was used as a cutoff for the low end of each factor loading.

To determine whether subjects' professional and role-oriented values changed over time and whether there were differences between stayers' and leavers' values, the various repeated measures designs as described above were repeated using subjects' factor scores retained from the NCS and RFS factor analyses. For each factor identified at $t_{1}$, a factor score was computed by summing the values obtained from multiplying the values of the variables loaded on the factor by their corresponding factor weight. After factor scores for each factor at $t_{1}$ were computed, these procedures were repeated, using the $t_{1}$ factor weights, for the values of the $t_{2}$ and $t_{3}$ variables in order to obtain their respective factor scores. To measure change in factor scores over the three time points, the factor scores for the NCS and the RFS were entered as the dependent variables in the 9x3 (aggregate) and the $2 \times 3$ (stayers versus leavers) RM-ANOVAs.

\section{$\underline{\text { Limitations }}$}

The limitations of this study pertain to three areas of concern. First, the study results are not generalizable beyond the accessible population at the medical center where the data were collected due to the self-selecting nature of the convenience sample. Subjects included in the study selected employment at the study site, and in turn their participation in the transition program. Second, there were substantial missing data, especially involving data collection at the third time point. Examination of the data and knowledge of the data collection methods reveal that this limitation resulted from a combination of subject attrition, declining retum rate of the questionnaires over time, and 
lapses in provisions for distribution and collection of questionnaires. Nevertheless, these factors caused not only uneven cell counts but also inadequate sample sizes with respect to the panel year groups, and consequently interfered with the planned statistical procedures. Third, although the changes in the transition program between 1987 and 1988 were relatively minor, the longitudinal nature of the study and thus its findings may be compromised by this disruption in protocols.

\section{Summary}

Utilizing secondary data, this study used repeated measures analysis of variance (RM-ANOVA) to examine the effect of a nurse transition program on neophyte staff nurses' clinical competency, role adjustment, job satisfaction, length of stay and turnover. RM-ANOVAs were also used to determine if, among new graduates participating in the ransition program, there were differences with regard to clinical competency, role adjustment, and job satisfaction between those who stayed beyond 15 months after hire (stayers) and those who terminated employment within 15 months (leavers). In addition, this study utilized exploratory factor analyses, with subsequent repeated measures designs based on the factor scores obtained from the FAs, in order to identify the nature and consequences of new graduates' professional and role-oriented values. 


\section{Chapter IV}

\section{RESULTS}

The purpose of this study was to assess whether, and to what degree, new graduate nurses' clinical competency, role adjustment, job satisfaction, and length of stay increase, and their rate of turnover decreases, as a result of their participation in a neophyte transition program. In addition, this study sought to determine if, among new graduates participating in the transition program, there are differences with regard to clinical competency, role adjustment, and job satisfaction between those who stay beyond 15 months after hire, and those who terminate employment within 15 months. Relatedly, this study sought to identify the professional and role-oriented values new nurses possessed upon initial employment, to determine whether these values changed over time, and to discern whether differing values affected the nurses' length of stay.

Secondary data, obtained using a longitudinal, panel study design, were analyzed using repeated measures analysis of variance and factor analysis. Sample description and the results of the statistical tests specific to the study's seven research questions are presented in this chapter.

\section{Sample Characteristics}

Panel Year Groups and the Aggregate Sample. The sample for this study consisted of 890 new graduate nurses hired as staff nurses by the hospital study site beginning in 1983 and ending in 1991. The demographic characteristics of the subjects in each panel year and the aggregate sample are provided in Table 2. As the table shows, the aggregate sample was composed primarily of women (94.3\%), who were 24 
Table 2

Demographic Characteristics for Each Panel Year and the Aggregate Sample

\begin{tabular}{|c|c|c|c|c|c|c|c|c|c|c|}
\hline Variable & $\begin{array}{c}1983 \\
(n=155) \\
\mathrm{f}(\%)\end{array}$ & $\begin{array}{c}1984 \\
(n=68) \\
f(\%)\end{array}$ & $\begin{array}{c}1985 \\
(n=110) \\
f(\%)\end{array}$ & $\begin{array}{c}1986 \\
(n=114) \\
f(\%)\end{array}$ & $\begin{array}{c}1987 \\
(n=93) \\
f(\%)\end{array}$ & $\begin{array}{c}1988 \\
(n=68) \\
\mathrm{f}(\%)\end{array}$ & $\begin{array}{c}1989 \\
(n=93) \\
f(\%)\end{array}$ & $\begin{array}{c}1990 \\
(n=91) \\
f(\%)\end{array}$ & $\begin{array}{c}1991 \\
(n=98) \\
\mathrm{f}(\%)\end{array}$ & $\begin{array}{c}\text { Total } \\
(n=890) \\
f(\%)\end{array}$ \\
\hline \multicolumn{11}{|l|}{ Age (years) } \\
\hline$\leq 24$ & $113(72.9)$ & $47(69.1)$ & $64(58.7)$ & $59(51.8)$ & $62(67.4)$ & $37(57.8)$ & $30(61.2)$ & $32(52.5)$ & $18(39.1)$ & $462(60.9)$ \\
\hline $25-29$ & $23(14.8)$ & $14(20.6)$ & $23(21.1)$ & $29(25.4)$ & $19(20.7)$ & $16(25.0)$ & $10(20.4)$ & $13(21.3)$ & $11(23.9)$ & $158(20.8)$ \\
\hline $30-34$ & $13(8.4)$ & $5(7.4)$ & $10(9.2)$ & $13(11.4)$ & $8(8.6)$ & $2(3.1)$ & $5(10.2)$ & $8(13.1)$ & $9(19.6)$ & $73(9.6)$ \\
\hline $35-39$ & $5 \quad(3.2)$ & $1(1.5)$ & $10(9.2)$ & $5(4.4)$ & $2(2.2)$ & $5(7.8)$ & $4(8.2)$ & $3(4.9)$ & $5(10.9)$ & $40(5.3)$ \\
\hline \multicolumn{11}{|l|}{ Gender } \\
\hline Female & $144(93.5)$ & $61(89.7)$ & $104(94.5)$ & $108(94.7)$ & $89(96.7)$ & $59(92.2)$ & $50(98.0)$ & $58(93.5)$ & $44(97.8)$ & 717 (94.3) \\
\hline Male & $10(6.5)$ & $7(10.3)$ & $6(5.5)$ & $6(5.3)$ & $3(3.3)$ & $5(7.8)$ & $1(2.0)$ & $4(6.5)$ & $1(2.2)$ & $43(5.7)$ \\
\hline \multicolumn{11}{|l|}{ Marital } \\
\hline \multicolumn{11}{|l|}{ Status } \\
\hline Single & $120(77.4)$ & $44(64.7)$ & $62(56.4)$ & $64(56.1)$ & $57(61.3)$ & $40(62.5)$ & $26(51.0)$ & $26(42.6)$ & $17(37.0)$ & $456(59.8)$ \\
\hline$<1 \mathrm{yr}$ & $5(3.2)$ & $5(7.4)$ & $9(8.2)$ & $8(7.0)$ & $7(7.5)$ & $7(10.9)$ & $6(11.8)$ & $5(8.2)$ & $2(4.3)$ & $54(7.1)$ \\
\hline$>1 \mathrm{yr}$ & $26(16.8)$ & $18(26.5)$ & $26(23.6)$ & $35(30.7)$ & $24(25.8)$ & $13(20.3)$ & $14(27.5)$ & $23(37.7)$ & $19(41.3)$ & $198(26.0)$ \\
\hline Divorced & 3 (1.9) & $0 \quad(0.0)$ & $8 \quad(7.3)$ & $2(1.8)$ & $4(4.3)$ & $4(6.3)$ & $4(7.8)$ & $6(9.8)$ & $5(10.9)$ & $36(4.7)$ \\
\hline \multicolumn{11}{|l|}{$\begin{array}{l}\text { Basic Nsg } \\
\text { Education }\end{array}$} \\
\hline$A D$ & $62(40.0)$ & $24(35.3)$ & $60(54.5)$ & $61(53.5)$ & $44(47.3)$ & $30(46.9)$ & $23(45.1)$ & $33(54.1)$ & $28(60.9)$ & 365 (47.9) \\
\hline Diploma & $6(3.9)$ & $2(2.9)$ & $8(7.3)$ & $8(7.0)$ & $1(1.1)$ & $1(1.6)$ & $1(2.0)$ & $1(1.6)$ & $1(2.2)$ & $29(3.8)$ \\
\hline BS & $87(56.1)$ & $42(61.8)$ & $42(38.2)$ & $45(39.5)$ & $48(51.6)$ & $33(51.6)$ & $27(52.9)$ & $27(44.3)$ & $17(37.0)$ & $368(48.3)$ \\
\hline Other & $0 \quad(0.0)$ & $0(0.0)$ & $0(0.0)$ & $0(0.0)$ & $0(0.0)$ & $0(0.0)$ & $0(0.0)$ & $0(0.0)$ & $0(0.0)$ & $0(0.0)$ \\
\hline
\end{tabular}




\begin{tabular}{|c|c|c|c|c|c|c|c|c|c|c|}
\hline Variable & $\begin{array}{c}1983 \\
(n=155) \\
f(\%)\end{array}$ & $\begin{array}{c}1984 \\
(n=68) \\
\mathrm{f}(\%)\end{array}$ & $\begin{array}{c}1985 \\
(n=110) \\
\mathrm{f}(\%)\end{array}$ & $\begin{array}{c}1986 \\
(n=114) \\
f(\%)\end{array}$ & $\begin{array}{c}1987 \\
(n=93) \\
\mathrm{f}(\%)\end{array}$ & $\begin{array}{c}1988 \\
(n=68) \\
f(\%)\end{array}$ & $\begin{array}{c}1989 \\
(n=93) \\
\text { f (\%) }\end{array}$ & $\begin{array}{c}1990 \\
(n=91) \\
\text { f }(\%)\end{array}$ & $\begin{array}{c}1991 \\
(n=98) \\
\mathrm{f}(\%)\end{array}$ & $\begin{array}{c}\text { Total } \\
(n=890) \\
f(\%)\end{array}$ \\
\hline \multicolumn{11}{|l|}{ Highest } \\
\hline \multicolumn{11}{|l|}{ Degree } \\
\hline \multicolumn{11}{|l|}{ Expected } \\
\hline BS & $50(32.5)$ & $15(22.1)$ & $40(36.4)$ & $48(42.1)$ & $26(28.3)$ & $17(27.0)$ & $17(33.3)$ & $16(26.2)$ & $16(34.8)$ & $245(32.3)$ \\
\hline MSN & $83(53.9)$ & $43(63.2)$ & $59(53.6)$ & $56(49.1)$ & $48(52.2)$ & $29(46.0)$ & $27(52.9)$ & $37(60.7)$ & $24(52.2)$ & 406 (53.5) \\
\hline Nsg PhD & 7 (4.5) & 4 (5.9) & $6(5.5)$ & $3(2.6)$ & $11(12.0)$ & $7(11.1)$ & $4(7.8)$ & $2(3.3)$ & $3(6.5)$ & $47(6.2)$ \\
\hline OtherPhD & $2(1.3)$ & $0(0.0)$ & $0(0.0)$ & $1(0.9)$ & $1(1.1)$ & $1(1.6)$ & $1(2.0)$ & $0(0.0)$ & $0(0.0)$ & $6(0.8)$ \\
\hline \multicolumn{11}{|l|}{ School } \\
\hline \multicolumn{11}{|l|}{ Employment } \\
\hline Full-time & $7(4.5)$ & $4(5.9)$ & $13(11.8)$ & $10(8.8)$ & $8(8.6)$ & $5(7.8)$ & $3(5.9)$ & $5 \quad(8.2)$ & $2(4.3)$ & $57(7.5)$ \\
\hline Part-time & $95(61.3)$ & $46(67.6)$ & $57(51.8)$ & $58(50.9)$ & $54(58.1)$ & $44(68.6)$ & $31(60.8)$ & $41(67.2)$ & $30(65.2)$ & $456(59.8)$ \\
\hline Summer & $26(16.8)$ & $8(11.8)$ & $14(12.7)$ & $16(14.0)$ & $18(19.4)$ & $5(7.8)$ & 7 (13.7) & $4(6.6)$ & $2(4.3)$ & $100(13.1)$ \\
\hline None & $26(16.8)$ & $9(13.2)$ & $25(22.7)$ & 27 (23.7) & $12(12.9)$ & 8 (12.5) & 8 (15.7) & $10(16.4)$ & $9(19.6)$ & $134(17.6)$ \\
\hline \multicolumn{11}{|l|}{ School } \\
\hline \multicolumn{11}{|l|}{$\begin{array}{l}\text { Employment } \\
\text { in Nursing }\end{array}$} \\
\hline Yes & $106(68.4)$ & $49(72.1)$ & 40 (36.7) & $49(43.4)$ & $63(69.2)$ & $42(66.7)$ & $40(78.4)$ & $45(73.8)$ & $28(62.2)$ & $462(61.1)$ \\
\hline No & 24 (15.5) & 10 (14.7) & $43(39.4)$ & 38 (33.6) & $18(19.8)$ & $13(20.6)$ & $3(5.9)$ & $6(9.8)$ & $8(17.8)$ & 163 (21.6) \\
\hline N/A & $25(16.1)$ & $9(13.2)$ & $26(23.9)$ & $26(23.0)$ & $10(11.0)$ & $8(12.7)$ & 8 (15.7) & $10(16.4)$ & $9(20.0)$ & $131(17.3)$ \\
\hline \multicolumn{11}{|l|}{$\begin{array}{l}\text { Experience } \\
\text { in hospital }\end{array}$} \\
\hline Nsg Asst & $66(42.6)$ & $26(38.2)$ & $35(31.8)$ & $28(24.6)$ & $36(38.7)$ & $15(22.1)$ & $21(22.6)$ & $32(35.2)$ & $5(5.1)$ & $264(29.7)$ \\
\hline LPN & 18 (11.6) & $5(7.4)$ & $10(9.1)$ & $14(12.3)$ & $19(20.4)$ & $8(11.8)$ & $10(10.8)$ & $10(11.0)$ & $11(11.2)$ & $105(11.8)$ \\
\hline Extem(s) & $44(28.4)$ & $32(47.1)$ & $1(0.9)$ & $10(8.8)$ & $13(14.0)$ & $18(26.5)$ & $15(16.1)$ & $18(19.8)$ & $6(6.1)$ & $157(17.6)$ \\
\hline Extem(o) & $27(17.4)$ & $4(5.9)$ & $4(3.6)$ & $14(12.3)$ & $9(9.7)$ & $3(4.4)$ & $7(7.5)$ & $2(2.2)$ & $3(3.1)$ & 73 (8.2) \\
\hline Other & $51(32.9)$ & $30(44.1)$ & 49 (44.5) & $44(38.6)$ & $27(29.0)$ & $25(36.8)$ & $16(17.2)$ & $20(22.0)$ & $12(12.2)$ & $274(30.8)$ \\
\hline
\end{tabular}




\begin{tabular}{|c|c|c|c|c|c|c|c|c|c|c|}
\hline Variable & $\begin{array}{c}1983 \\
(n=155) \\
\mathrm{f}(\%)\end{array}$ & $\begin{array}{c}1984 \\
(n=68) \\
f(\%)\end{array}$ & $\begin{array}{c}1985 \\
(n=110) \\
f(\%)\end{array}$ & $\begin{array}{c}1986 \\
(n=114) \\
f(\%)\end{array}$ & $\begin{array}{c}1987 \\
(n=93) \\
f(\%)\end{array}$ & $\begin{array}{c}1988 \\
(n=68) \\
f(\%)\end{array}$ & $\begin{array}{c}1989 \\
(n=93) \\
f(\%)\end{array}$ & $\begin{array}{c}1990 \\
(n=91) \\
f(\%)\end{array}$ & $\begin{array}{c}1991 \\
(n=98) \\
\mathrm{f}(\%)\end{array}$ & $\begin{array}{c}\text { Total } \\
(n=890) \\
f(\%)\end{array}$ \\
\hline \multicolumn{11}{|l|}{ Clinical } \\
\hline \multicolumn{11}{|l|}{ Interest } \\
\hline ICU/ER & $42(27.1)$ & $19(27.9)$ & $31(28.2)$ & $31(27.2)$ & $28(30.1)$ & $20(29.4)$ & $15(16.1)$ & $21(23.1)$ & $14(14.3)$ & $221(24.8)$ \\
\hline Medical & $20(12.9)$ & $14(20.6)$ & $13(11.8)$ & $14(12.3)$ & $8(8.6)$ & $6(8.8)$ & $4(4.3)$ & $5(5.5)$ & $7(7.1)$ & $91(10.2)$ \\
\hline Surgical & $25(16.1)$ & $9(13.2)$ & $13(11.8)$ & $13(11.4)$ & $19(20.4)$ & $6(8.8)$ & 9 (9.7) & $7(7.7)$ & $10(10.2)$ & $111(12.5)$ \\
\hline Pediatrics & $29(18.7)$ & $7(10.3)$ & $13(11.8)$ & $22(19.3)$ & $16(17.2)$ & $6(8.8)$ & $11(11.8)$ & $3(3.3)$ & $2(2.0)$ & 109 (12.2) \\
\hline Maternity & $17(11.0)$ & $14(20.6)$ & $23(20.9)$ & $18(15.8)$ & $15(16.1)$ & $13(19.1)$ & $6(6.5)$ & $12(13.2)$ & $5(5.1)$ & $123(13.8)$ \\
\hline Psych & $5(3.2)$ & $0(0.0)$ & $4(3.6)$ & $1(0.9)$ & $4(4.3)$ & $7(10.3)$ & $1(1.1)$ & $7(7.7)$ & $3(3.1)$ & $32(3.6)$ \\
\hline Rehab & $2(1.3)$ & $0(0.0)$ & $4(3.6)$ & $3(2.6)$ & $0(0.0)$ & $2(2.9)$ & $1(1.1)$ & $1(1.1)$ & $0(0.0)$ & $13(1.5)$ \\
\hline Geriatrics & $6(3.9)$ & $0 \quad(0.0)$ & $0(0.0)$ & $4(3.5)$ & $1(1.1)$ & $0 \quad(0.0)$ & $0(0.0)$ & $0(0.0)$ & $1(1.0)$ & $12(1.3)$ \\
\hline Commnty & $1(0.6)$ & $2(2.9)$ & $6(5.5)$ & $4 \quad(3.5)$ & $0 \quad(0.0)$ & $0(0.0)$ & $1(1.1)$ & $0(0.0)$ & $1(1.0)$ & $15(1.7)$ \\
\hline Other & $8(5.2)$ & $3(4.4)$ & $3(2.7)$ & $4 \quad(3.5)$ & $2(2.2)$ & $8(11.8)$ & $45(48.4)$ & $35(38.5)$ & $55(56.1)$ & $163(18.3)$ \\
\hline \multicolumn{11}{|l|}{ Hospital } \\
\hline \multicolumn{11}{|l|}{ Practice } \\
\hline \multicolumn{11}{|l|}{ Intent } \\
\hline$<1 \mathrm{yr}$ & $2(1.3)$ & $0 \quad(0.0)$ & $0(0.0)$ & $0(0.0)$ & $0(0.0)$ & $1(1.6)$ & $1(2.0)$ & 1 (1.7) & $1(2.2)$ & $6(0.8)$ \\
\hline $1-3$ yrs & $25(16.1)$ & $12(17.9)$ & $17(15.5)$ & $20(17.5)$ & $13(14.1)$ & $9(14.3)$ & $4(7.8)$ & $14(23.7)$ & 7 (15.6) & $121(16.0)$ \\
\hline $4-10$ yrs & $73(47.1)$ & $34(50.7)$ & $44(40.0)$ & $53(46.5)$ & $51(55.4)$ & $26(41.3)$ & $31(60.8)$ & $25(42.4)$ & $17(37.8)$ & $354(46.8)$ \\
\hline Always & $48(31.0)$ & $19(28.4)$ & $45(40.9)$ & $38(33.3)$ & $25(27.2)$ & $20(31.7)$ & $12(23.5)$ & $19(32.2)$ & $19(42.2)$ & $245(32.4)$ \\
\hline Other & $7(4.5)$ & $2(3.0)$ & $4(3.6)$ & $3(2.6)$ & $3(3.3)$ & 7 (11.1) & $3(5.9)$ & $0(0.0)$ & $1(2.2)$ & $30(4.0)$ \\
\hline \multicolumn{11}{|l|}{$\begin{array}{l}\text { Why this } \\
\text { hospital? }\end{array}$} \\
\hline Familiar & $21(13.6)$ & $12(17.9)$ & 10 & $8 \quad(7.0)$ & $17(18.5)$ & $13(20.3)$ & $9(17.6)$ & 9 (14.8) & $8(17.4)$ & $107(14.1)$ \\
\hline Pay & 3 (1.9) & $0(0.0)$ & $1(0.9)$ & $2(1.8)$ & $3(3.3)$ & $1(1.6)$ & $3(5.9)$ & $2(3.3)$ & $3(6.5)$ & $18(2.4)$ \\
\hline Cont Ed & $37(24.0)$ & $11(16.4)$ & $27(24.5)$ & $31(27.2)$ & $22(23.9)$ & $7(10.9)$ & $10(19.6)$ & $10(16.4)$ & $12(26.1)$ & $167(22.0)$ \\
\hline Near Hm & $12(7.8)$ & $3(4.5)$ & $8(7.3)$ & $10(8.8)$ & $7(7.6)$ & 7 (10.9) & $4(7.8)$ & $6(9.8)$ & $3(6.5)$ & $60(7.9)$ \\
\hline Med Ctr & $30(19.5)$ & $21(31.3)$ & $33(30.0)$ & $31(27.2)$ & $16(17.4)$ & 14 (21.9) & $12(23.5)$ & $9(14.8)$ & $8(17.4)$ & 174 (22.9) \\
\hline Sig Other & $10(6.5)$ & 8 (11.9) & $2(1.8)$ & $9(7.9)$ & $5(5.4)$ & $6(9.4)$ & $5(9.8)$ & 4 (6.6) & $3(6.5)$ & $52(6.9)$ \\
\hline Wk Envir & $33(21.4)$ & $9(13.4)$ & $24(21.8)$ & $19(16.7)$ & $19(20.7)$ & $10(15.6)$ & $6(11.8)$ & $14(23.0)$ & $4(8.7)$ & $138(18.2)$ \\
\hline Other & $8(5.2)$ & $3(4.5)$ & $5(4.5)$ & $4(3.5)$ & $3(3.3)$ & $6(9.4)$ & $2(3.9)$ & 7 (11.5) & $5(10.9)$ & $43(5.7)$ \\
\hline
\end{tabular}


years or younger $(60.9 \%)$ in age, and single $(59.8 \%)$. The sample was essentially evenly divided with regard to basic nursing education, that is $47.9 \%$ of the subjects had an associate degree and $48.3 \%$ reported baccalaureate preparation. A significant majority of the subjects also indicated that they expected to pursue additional education, with over half (53.5\%) reporting that they intended to obtain an MSN. In addition, the majority of subjects had worked part-time $(59.8 \%)$ in nursing positions $(61.1 \%)$ while in nursing school and identified the intensive care unit and emergency room as their areas of clinical interest. Of particular interest to this study's research questions concerning length of stay and turnover is the fact that while almost $48 \%$ of the newly graduated nurses intended to work in a hospital setting from four to ten years, $16.8 \%$ of the subjects indicated an intent to work in a hospital setting less than three years. And considering this study's inquiry about nurses' values, it is interesting to note that the top four reasons given for selection of the study site hospital for employment were: developing medical center (22.9\%); opportunity to continue education (22\%); positive work environment (18.2\%); and familiar territory (14.1\%).

Chi-square analyses were conducted on the demographic variables to determine whether the nine panel groups were drawn from the same population and thus whether the aggregate sample was homogenous with respect to the demographic characteristics. Results of these analyses are provided in Table 3. As the table indicates, there were no significant differences between the nine groups with regard to gender, highest degree expected, school employment, experience in the hospital, length of time intending to work in a hospital setting, and reasons for selecting the study site hospital for 
Table 3

Significance of Differences on the Demographic Variables for the Panel Groups

\begin{tabular}{lccc}
\hline Variable & $X^{2}$ & $d f$ & $p<$ \\
\hline Age & 57.52 & 32 & .004 \\
Sex & 6.90 & 8 & --- \\
Marital Status & 67.98 & 32 & .0002 \\
Basic Nursing Education & 29.85 & 16 & .019 \\
Highest Degree Expected & 34.51 & 32 & --- \\
School Employment & 38.04 & 32 & --- \\
Employment in Nursing & 71.41 & 16 & .0001 \\
Experience in Hospital & 8.94 & 32 & --- \\
Clinical Interest & 352.55 & 72 & .0001 \\
Hospital Practice Intent & 36.48 & 32 &.-- \\
Why this Hospital & 66.05 & 56 & --- \\
\end{tabular}

employment. However, statistically significant differences were found between these panel groups concerning age, marital status, basic nursing education, school employment in nursing, and clinical interest area. These differences can be explained by the missing data in the last three panel groups (i.e., 1989, 1990, 1991); and by the higher proportion of total subjects in the first panel group (i.e., 1983) which created a higher than expected frequency count in various cells for that group across the demographic variables. Since the demographic characteristics of the first panel group were still representative of a new 
graduate profile, these differences were deemed to be negligible and thus it was concluded that the nine panel groups were drawn from the same population and that the aggregate sample was homogenous with respect to demographic characteristics.

Cross-sectional analyses of variance were also performed on data collected at entry $\left(t_{1}\right)$ with respect to the outcome variables to determine whether the nine panel groups significantly differ on these measures prior to their participation in the transition program and thus whether the aggregate sample was homogenous with respect to these variables. One-way ANOVAs were planned to allow for the inclusion of post hoc tests. However, evaluation of the data indicated that although the ANOVA assumption of independence of observations was met, examination of the variables' distributions revealed numerous violations of the assumption of normality. In addition, the values for Cochrans $\mathrm{C}$ and Bartlett-Box $\mathrm{F}$ indicated violation of the assumption of homogeneity of variance. In light of these violations, the one-way ANOVA's nonparametric equivalent, the Kruskal-Wallis test, are reported.

Table 4 summarizes the measures of central tendency and the results of the Kruskal-Wallis cross-sectional analyses for the nine panel groups with regard to subjects' mean scores on the outcome variables at $t_{1}$. As the table shows, there were significant differences between the groups on the Medications and the Professionalization subscales of the NCA, the NCS total, the RFS total, and the JSI total, as well as three of the JSI's subscales--Intrinsic, Interpersonal, and Extrinsic. Post hoc tests revealed that these differences were primarily due to the first group's (1983) lower mean scores on these outcome variables as compared to other groups' means. It was concluded, therefore, that 
Table 4

Measures of Central Tendency and Dispersion and Results of Cross-Sectional Analyses on the Outcome Variables for the Panel Groups at the First Time Point

\begin{tabular}{|c|c|c|c|c|c|c|c|c|c|c|c|c|c|c|c|c|c|c|c|c|}
\hline \multirow{2}{*}{ Variable } & \multicolumn{2}{|c|}{1983} & \multicolumn{2}{|c|}{1984} & \multicolumn{2}{|c|}{1985} & \multicolumn{2}{|c|}{1986} & \multicolumn{2}{|c|}{1987} & \multicolumn{2}{|c|}{1988} & \multicolumn{2}{|c|}{1989} & \multicolumn{2}{|c|}{1990} & \multicolumn{2}{|c|}{1991} & \multirow{2}{*}{$x^{2}$} & \multirow[b]{2}{*}{$p<$} \\
\hline & M & $\boldsymbol{D}$ & M & $\boldsymbol{D}$ & $M$ & $\boldsymbol{D}$ & M & $\Phi$ & M & D & $M$ & $\boldsymbol{D}$ & M & $\boldsymbol{D}$ & $M$ & D & $M$ & $\boldsymbol{D}$ & & \\
\hline NCA Tored & 105.47 & 21.17 & 110.36 & 12.83 & 110.88 & 13.19 & 109.29 & 13.80 & 113.59 & 12.84 & 110.45 & 14.04 & 111.37 & 13.90 & 100.71 & 14.11 & 104.07 & 19.19 & 13.64 & - \\
\hline 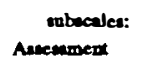 & 23.64 & 4.44 & 24.18 & 3.63 & 23.89 & 3.89 & 24.44 & 3.71 & 24.60 & 3.47 & 23.54 & 4.04 & 25.12 & 4.08 & 23.62 & 3.99 & 23.19 & 4.07 & 10.75 & -. \\
\hline Inervertion & 13.45 & 3.02 & 14.08 & 2.46 & 14.01 & 2.26 & 13.88 & 2.51 & 14.17 & 2.36 & 14.28 & 2.57 & 14.10 & 2.18 & 13.87 & 2.82 & 13.20 & 3.73 & 7.16 & - \\
\hline Modicasions & 6.63 & 1.53 & 6.76 & 1.35 & 7.20 & 1.31 & 7.04 & 1.43 & 7.56 & 1.38 & 6.92 & 1.82 & 7.02 & 1.44 & 6.87 & 1.56 & 6.96 & 1.46 & 26.20 & .001 \\
\hline Riuk Mgresx & 11.38 & 2.96 & 12.02 & 1.69 & 11.95 & 1.71 & 11.84 & 1.80 & 12.40 & 1.74 & 12.28 & 2.00 & 11.75 & 1.95 & 11.73 & 2.07 & 11.50 & 2.47 & 9.48 & - \\
\hline Poxienx Suppon & 19.30 & 5.08 & 20.63 & 2.67 & 20.83 & 2.50 & 20.05 & 2.98 & 20.69 & 2.87 & 20.86 & 2.52 & 20.28 & 2.82 & 19.60 & 2.94 & 19.73 & 3.20 & 10.29 & .. \\
\hline Profescioaral & 20.66 & 4.62 & 21.42 & 3.33 & 21.97 & 3.46 & 20.98 & 3.79 & 20.2 & 3.69 & 2.21 & 3.29 & 22.04 & 3.52 & 21.20 & 3.44 & 20.52 & 4.30 & 23.20 & .003 \\
\hline NCS Tord & 49.17 & 7.98 & 49.97 & 5.01 & 53.13 & 5.15 & 52.14 & 4.64 & 52.36 & 5.65 & 52.17 & 6.05 & 52.7 & $4 . \pi$ & 52.98 & 6.19 & 53.63 & 6.00 & 33.98 & .0001 \\
\hline RFS Toral & 73.09 & 10.49 & 73.97 & 9.59 & 2.27 & 10.98 & 71.59 & 11.40 & 75.40 & 8.49 & 74.41 & 11.50 & 72.33 & 11.14 & 0.18 & 10.43 & 66.89 & 14.90 & 18.56 & .02 \\
\hline JSI Toral & 57.63 & 8.97 & 60.40 & 8.89 & 62.62 & 9.03 & 02.87 & 8.95 & 60.46 & 7.88 & 58.12 & 7.94 & 63.25 & 7.14 & 63.71 & 7.91 & 63.55 & 9.20 & 29.00 & .0003 \\
\hline Involvemest & 13.98 & 2.76 & 15.02 & 2.61 & 14.86 & 2.86 & 15.15 & 2.48 & 14.67 & 2.04 & 14.56 & 1.92 & 15.12 & 2.54 & 14.56 & 2.85 & 15.43 & 2.50 & 12.62 & - \\
\hline Imerpernosal & 15.36 & 2.84 & 16.35 & 2.86 & 16.75 & 2.49 & 16.93 & 2.44 & 16.43 & 2.57 & 16.22 & 3.06 & 17.06 & 2.00 & 16.85 & 2.09 & 16.65 & 2.62 & 2.41 & .004 \\
\hline Exrineic & 13.43 & 2.58 & 13.50 & 2.61 & 15.00 & 2.34 & 14.65 & 2.83 & 13.49 & 3.02 & 13.35 & 2.67 & 15.23 & 1.82 & 15.15 & 2.51 & 15.16 & 2.40 & 38.02 & .0001 \\
\hline
\end{tabular}


the nine panel groups were not homogenous upon entry into the transition program with respect to the variables measuring clinical competency, role adjustment, and job satisfaction.

Stayers and Leavers. The demographic characteristics for the two groups stayers and leavers are provided in Table 5. As can be seen from this table, and also when compared to the descriptive information in Table 2, these two groups portray the same overall characteristics as the aggregate sample with respect to the demographic variables.

Table 5

Demographic Characteristics and Significance of Differences for Stayers and Leavers

\begin{tabular}{|c|c|c|c|c|c|}
\hline Variable & $\begin{array}{c}\text { Stayers } \\
n=583 \\
\mathbf{f}(\%)\end{array}$ & $\begin{array}{c}\text { Leavers } \\
\begin{array}{c}n=306 \\
\mathrm{f}(\%)\end{array}\end{array}$ & $X^{2}$ & $d f$ & $p<$ \\
\hline \multicolumn{6}{|l|}{ Age (years) } \\
\hline$\leq 24$ & $290(59.7)$ & $172(63.2)$ & 3.95 & 4 & - \\
\hline $25-29$ & $106(21.8)$ & $52(19.1)$ & & & \\
\hline $30-34$ & $45(9.3)$ & $28(10.3)$ & & & \\
\hline $35-39$ & $25(5.1)$ & $15(5.5)$ & & & \\
\hline 240 & $20(4.1)$ & $5(1.8)$ & & & \\
\hline \multicolumn{6}{|l|}{ Gender } \\
\hline Female & $454(93.8)$ & $263(95.3)$ & .73 & 1 & - \\
\hline Male & $30(6.2)$ & $13(4.7)$ & & & \\
\hline \multicolumn{6}{|l|}{ Marital Status } \\
\hline Single & $280(57.6)$ & $176(63.8)$ & 5.88 & 4 & - \\
\hline Married < $1 \mathrm{yr}$ & $32(6.6)$ & $22(8.0)$ & & & \\
\hline Married >1 yr & $140(28.8)$ & $58(21.0)$ & & & \\
\hline Separated & $12(2.5)$ & $6(2.2)$ & & & \\
\hline Divorced & $22(4.5)$ & $14(5.1)$ & & & \\
\hline \multicolumn{6}{|l|}{ Basic Nsg Education } \\
\hline$A D$ & $246(50.6)$ & $119(43.1)$ & 4.01 & 2 & - \\
\hline Diploma & $17(3.5)$ & $12(4.3)$ & & & \\
\hline BS & $223(45.9)$ & $145(52.5)$ & & & \\
\hline Other & $0(0.0)$ & $0(0.0)$ & & & \\
\hline \multicolumn{6}{|c|}{ Highest Degroe Expected } \\
\hline BS & $161(33.3)$ & $84(30.4)$ & .94 & 4 & - \\
\hline MSN & $253(52.4)$ & $153(55.4)$ & & & \\
\hline MS & $36(7.5)$ & $19(6.9)$ & & & \\
\hline Nsg Doctorate & $29(6.0)$ & $18(6.5)$ & & & \\
\hline Other Doctorate & $4(0.8)$ & $2(0.7)$ & & & \\
\hline
\end{tabular}




\begin{tabular}{|c|c|c|c|c|c|}
\hline Variable & $\begin{array}{c}\text { Stayers } \\
n=583 \\
\mathrm{f}(\%)\end{array}$ & $\begin{array}{c}\text { Leavers } \\
n=306 \\
\mathrm{f}(\%)\end{array}$ & $X^{2}$ & $d f$ & $p<$ \\
\hline \multicolumn{6}{|l|}{ School Employment } \\
\hline Full-time & $40(8.2)$ & $17(6.2)$ & 8.74 & 4 & - \\
\hline Part-time & $290(59.7)$ & $166(60.1)$ & & & \\
\hline Summer & $53(10.9)$ & $47(17.0)$ & & & \\
\hline None & $91(18.7)$ & $43(15.6)$ & & & \\
\hline Other & $12(2.5)$ & $3(1.1)$ & & & \\
\hline \multicolumn{6}{|c|}{ School Employment in Nursing } \\
\hline Yes & $293(60.7)$ & $169(61.9)$ & 1.82 & 2 & - \\
\hline No & $100(20.7)$ & $63(23.1)$ & & & \\
\hline N/A & $90(18.6)$ & $41(15.0)$ & & & \\
\hline \multicolumn{6}{|l|}{ Experience in hospital } \\
\hline Nsg Asst & $168(28.8)$ & $96(31.4)$ & .63 & 4 & - \\
\hline LPN & $71(12.2)$ & $34(11.1)$ & & & \\
\hline Extern (study site) & $100(17.2)$ & $57(18.6)$ & & & \\
\hline Extern (other) & $44(7.5)$ & $29(9.5)$ & & & \\
\hline Other & $183(31.4)$ & 91 (29.7) & & & \\
\hline \multicolumn{6}{|l|}{ Clinical Interest } \\
\hline ICU/ER & $150(25.7)$ & $71(23.2)$ & 14.20 & 9 & - \\
\hline Medical & $58(9.9)$ & $33(10.8)$ & & & \\
\hline Surgical & $66(11.3)$ & 45 (14.7) & & & \\
\hline Pediatrics & $67(11.5)$ & $42(13.7)$ & & & \\
\hline Matemity & $77(13.2)$ & $46(15.0)$ & & & \\
\hline Psychiatry & $24(4.1)$ & $8(2.6)$ & & & \\
\hline Rehabilitation & $10(1.7)$ & $3(1.0)$ & & & \\
\hline Geriatrics & $7(1.2)$ & $5(1.6)$ & & & \\
\hline Community & $6(1.0)$ & $9(2.9)$ & & & \\
\hline Other & $118(20.2)$ & $44(14.4)$ & & & \\
\hline \multicolumn{6}{|l|}{ Hospital Practice Intent } \\
\hline$<1 \mathrm{yr}$ & $1(0.2)$ & $5(1.8)$ & 10.80 & 4 & .029 \\
\hline $1-3$ yrs & $85(17.7)$ & $36(13.1)$ & & & \\
\hline 4-10 yrs & $229(47.6)$ & $125(45.5)$ & & & \\
\hline Always & $145(30.1)$ & $100(36.4)$ & & & \\
\hline Other & $21(4.4)$ & $9(3.3)$ & & & \\
\hline \multicolumn{6}{|l|}{ Why this hospital? } \\
\hline Familiar Territory & $61(12.6)$ & $46(16.8)$ & 4.95 & 7 & - \\
\hline Pay & $13(2.7)$ & $5(1.8)$ & & & \\
\hline Cont Education Opport. & $106(21.9)$ & $61(22.3)$ & & & \\
\hline Near Home & $38(7.8)$ & $22(8.0)$ & & & \\
\hline Medical Center & $111(22.9)$ & $63(23.0)$ & & & \\
\hline Near Significant Other & $34(5.8)$ & $18(6.6)$ & & & \\
\hline Work Environment & $96(19.8)$ & $42(15.3)$ & & & \\
\hline Other & $26(5.4)$ & $17(6.2)$ & & & \\
\hline
\end{tabular}

Table 5 also contains the results of the chi-square analyses performed on the groups designated as stayers and leavers to determine if there were demographic 
differences between the two groups. As shown in the table, stayers and leavers did not differ significantly with respect to any of the demographic variables except the length of time they intended to work in a hospital setting. On this one characteristic, a higher proportion of stayers indicated an intent to remain in hospital nursing in the categories of 1 to 3 years and 4 to 10 years, while a higher proportion of leavers reported an intent to always work in a hospital setting. Of note also, is the finding that a higher proportion of leavers than stayers indicated an intent to work in a hospital setting for less than one year, $1.8 \%$ and $0.2 \%$ respectively. Actual length of stay (LOS) data for these two groups support these differences. Nurses in the stayers group had a considerably longer average LOS $(M=47.58$ months, $m d n=40.00, S D=28.87)$ than nurses in the leavers group $(M=3.85$ months, $m d n=5.71, S D=4.97)$.

Research Question 1: Do neophyte nurses participating in the transition program demonstrate a significant increase in their clinical competency, professional role adjustment, and job satisfaction? Table 6 presents descriptive summary statistics for the nine panel groups on the outcome variables at each of the three time points. As shown in the table, new graduates across the groups demonstrated a moderate level of proficiency upon hire with regard to their overall clinical competency (NCA) and their specific competencies (NCA subscales). Except for the 1988 group which had no data at the third time point for this instrument, subjects in the panel groups also demonstrated an increase in mean scores on the NCA total across all three time points with mean scores at $t_{3}$ indicating a moderately high level of clinical proficiency. In addition, subjects' mean scores on the NCA subscales pertaining to Intervention, Documentation, 
Table 6

Measures of Central Tendency and Dispersion on the Outcome Variables for the Panel Groups and the Aggregate Sample at Each Time Point

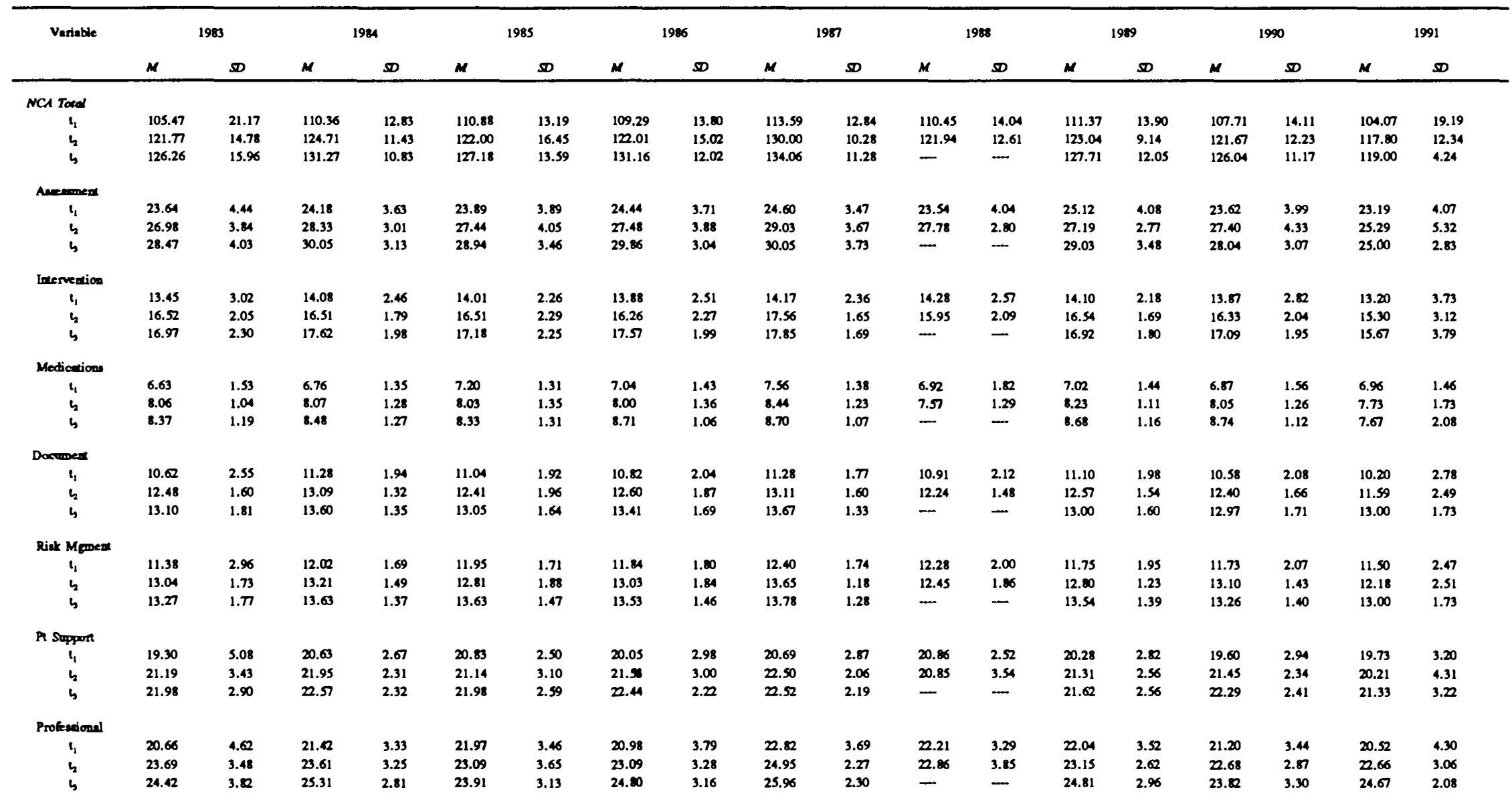




\begin{tabular}{|c|c|c|c|c|c|c|c|c|c|c|c|c|c|c|c|c|c|c|}
\hline \multirow[t]{2}{*}{ Veriable } & \multicolumn{2}{|c|}{1983} & \multicolumn{2}{|c|}{1984} & \multicolumn{2}{|c|}{1985} & \multicolumn{2}{|c|}{1986} & \multicolumn{2}{|c|}{1987} & \multicolumn{2}{|c|}{1988} & \multicolumn{2}{|c|}{1989} & \multicolumn{2}{|c|}{1990} & \multicolumn{2}{|c|}{1991} \\
\hline & M & $\boldsymbol{D}$ & M & D & M & $\boldsymbol{D}$ & $M$ & $\boldsymbol{D}$ & $M$ & $\boldsymbol{D}$ & $M$ & $\boldsymbol{D}$ & M & $\boldsymbol{D}$ & M & D & $M$ & $\boldsymbol{D}$ \\
\hline \multicolumn{19}{|l|}{ NCS Toral } \\
\hline $4_{1}$ & 49.17 & 7.98 & 49.97 & 3.01 & 53.13 & 5.15 & 52.14 & 4.64 & 52.36 & 5.65 & 52.17 & 6.05 & 52.7 & 4.7 & 52.98 & 6.19 & 53.60 & 6.00 \\
\hline is & 90.72 & 5.22 & 48.91 & 5.13 & 49.20 & 6.97 & 47.97 & 6.87 & 48.22 & 6.49 & 47.05 & 6.78 & 49.14 & 6.76 & 50.72 & 7.31 & 52.05 & 7.61 \\
\hline b & 48.54 & 7.05 & 47.12 & 6.87 & 49.19 & 6.01 & 47.35 & 8.09 & 48.46 & 8.17 & - & - & 50.20 & 5.71 & 52.36 & 6.68 & 5.33 & 8.39 \\
\hline \multicolumn{19}{|l|}{ MFS Toral } \\
\hline$l_{1}$ & 73.09 & 10.49 & 73.97 & 9.59 & 72.27 & 10.98 & 71.59 & 11.40 & 75.40 & 8.49 & 74.41 & 11.50 & 72.33 & 11.14 & 70.18 & 10.43 & 66.89 & 14.90 \\
\hline \& & 81.66 & 8.79 & 79.67 & 9.17 & $\pi .15$ & 10.39 & 79.82 & 11.44 & $\$ 0.31$ & 8.55 & 8.52 & 8.61 & 0.09 & 8.91 & 75.70 & 8.57 & 74.84 & 9.17 \\
\hline is & 84.25 & 9.99 & 82.88 & 9.44 & 82.03 & 9.80 & 85.02 & 8.93 & 87.27 & 8.59 & - & - & 83.29 & 9.70 & 81.36 & 9.47 & 83.67 & 6.35 \\
\hline \multicolumn{19}{|l|}{ JSI Toral } \\
\hline$l_{1}$ & 5.03 & 8.97 & 60.40 & 8.89 & 62.62 & 9.03 & 02.87 & 8.95 & 60.46 & 7.88 & 38.12 & 7.94 & 63.25 & 2014 & 63.71 & 7.97 & 63.55 & 9.20 \\
\hline is & 60.06 & 7.61 & 60.19 & 7.27 & 60.23 & 8.04 & 61.07 & 9.62 & 60.35 & 6.59 & 7.15 & 7.54 & 60.52 & 7.68 & 59.69 & 10.8 & 59.97 & 9.06 \\
\hline$b^{2}$ & 38.64 & 7.49 & 5.07 & 9.71 & 60.21 & 8.29 & $\$ 9.17$ & 8.82 & 59.77 & 7.94 & 65.33 & 5.03 & 60.70 & 6.28 & 60.37 & 10.13 & $\ldots$ & - \\
\hline \multicolumn{19}{|l|}{ Imerineic } \\
\hline i & 14.98 & 2.02 & 15.48 & 2.70 & 16.11 & 2.48 & 16.15 & $2 . \pi$ & 15.94 & 2.14 & 14.17 & 2.50 & 16.31 & 2.32 & 16.48 & 2.39 & 16.52 & 2.78 \\
\hline is & 15.60 & 2.32 & 15.37 & 2.26 & 15.41 & 2.33 & 15.81 & 2.56 & 15.66 & 2.20 & 14.55 & 2.50 & 15.94 & 2.47 & 15.42 & 3.17 & 15.55 & 2.61 \\
\hline b & 15.43 & 2.20 & 14.91 & 2.60 & 15.60 & 2.5 & 15.00 & 2.95 & 15.40 & 2.39 & 15.67 & 2.08 & 15.95 & 2.13 & 15.54 & 2.90 & - & - \\
\hline \multicolumn{19}{|l|}{ Irvolvememt } \\
\hline$t_{1}$ & 13.98 & 2.76 & 15.02 & 2.61 & 14.86 & 2.86 & 15.15 & 2.48 & 14.67 & 2.04 & 14.56 & 1.92 & 15.12 & 2.54 & 14.56 & 2.85 & 15.43 & 2.50 \\
\hline$b_{4}$ & 15.04 & 2.25 & 14.79 & 2.35 & 14.81 & 2.52 & 15.03 & 3.15 & 15.30 & 1.97 & 14.33 & 2.27 & 15.03 & 2.21 & 14.29 & 3.17 & 14.91 & 2.49 \\
\hline b & 15.20 & 2.05 & 14.61 & 2.58 & 15.12 & 2.59 & 14.80 & 2.70 & 15.13 & 2.05 & 15.67 & 1.53 & 15.05 & 2.03 & 14.63 & 3.01 & - & - \\
\hline \multicolumn{19}{|c|}{ Ioteperionel } \\
\hline$t_{1}$ & 15.36 & 2.84 & 16.35 & 2.86 & 16.75 & 2.49 & 16.93 & 2.44 & 16.43 & 2.57 & 16.22 & 3.06 & 17.06 & 2.00 & 16.85 & 2.09 & 16.65 & 2.62 \\
\hline 4 & 16.16 & 2.10 & 16.37 & 2.07 & 15.93 & 2.49 & 16.11 & 2.67 & 16.84 & 1.99 & 15.6 & 2.24 & 15.79 & 1.94 & 16.22 & 2.7 & 15.48 & 2.90 \\
\hline$b$ & 15.66 & 2.33 & 15.14 & 2.81 & 15.63 & 2.40 & 15.94 & 2.66 & 16.40 & 2.30 & 19.00 & 1.73 & 15.97 & 1.99 & 15.60 & 3.29 & - & - \\
\hline \multicolumn{19}{|l|}{ Extrinsic } \\
\hline$t_{1}$ & 13.43 & 2.58 & 13.50 & 2.61 & 15.00 & 2.34 & 14.65 & 2.83 & 13.49 & 3.02 & 13.35 & 2.67 & 15.23 & 1.82 & 15.15 & 2.51 & 15.16 & 2.40 \\
\hline 4 & 13.00 & 2.74 & 13.65 & 2.62 & 14.26 & 2.40 & 14.03 & 2.63 & 12.76 & 2.75 & 12.91 & 2.53 & 13.67 & 2.70 & 13.81 & 2.89 & 14.05 & 2.59 \\
\hline b & 12.32 & 2.55 & 12.63 & 3.11 & 13.86 & 2.28 & 13.21 & 2.54 & 12.83 & 2.74 & 15.00 & 1.00 & 13.50 & 2.38 & 14.60 & 2.7 & $\ldots$ & $\ldots$ \\
\hline
\end{tabular}

Nole: Doted lines for the 1988 and 1991 group inticere mising dea for the time point. 
Risk Management, and Professionalization showed consistent increases across the three time points and across all groups. However, 1991 subjects' mean scores on the NCA subscales for Assessment and Medications decreased between the second and third time point; and the 1988 group showed a decrease in mean scores between the first and second time point on the Patient Support subscale.

Professional role adjustment takes into account subjects' responses on both the Nursing Characteristics Scale (NCS) and the Role Function Survey (RFS). As the NCS scores in Table 6 show, the new graduates generally displayed a moderately positive perception of nursing practice both upon employment and after one year on the job. This perception of nursing practice was quite consistent across the nine groups. In contrast, there was a large degree of variability in the groups' changes in mean scores on the NCS over time. Subjects in the 1983 group demonstrated an increase in their mean scores on the NCS from $t_{1}$ to $t_{2}$, followed by a decrease in mean scores from $t_{2}$ to $t_{3}$. The mean scores for new graduates in the $1984,1985,1986$, and 1988 groups, however, displayed a consistent decline over the three time points. And mean scores for subjects in the remaining groups $(1987,1989,1990,1991)$ decreased between the first and second time points, but increased between the second and third time points.

With regard to the RFS scores, new graduates across the nine groups reported a moderately high degree of confidence in their familiarity with work demands and the hospital environment at the time of employment. This degree of confidence consistently improved over time for all groups as indicated by the increase in subjects' mean scores on the RFS (see Table 6). 
Job satisfaction scores (JSI) for subjects in the study indicated a moderately high degree of overall satisfaction across the groups upon hire and after one year of employment (see JSI total, Table 6). However, similar to subjects' scores on the NCS, there was large variability in the manner with which mean scores changed over time. New graduates in the 1983 group reported an increase in their mean scores on the JSI from $t_{1}$ to $t_{2}$, followed by a decrease in mean scores from $t_{2}$ to $t_{3}$. The mean scores for subjects in the 1984, 1985, 1986, 1987, and 1991 groups, however, displayed a consistent decline over the three time points. And mean scores for respondents in the other groups $(1988,1989,1990)$ decreased between the first and second time points, but increased from the second to the third time points. On the JSI's subscales, an increase in mean scores from $t_{1}$ to $t_{2}$ with a decrease from $t_{2}$ to $t_{3}$ were reported as follows (scale/panel group): Intrinsic/1983; Involvement/1987; Interpersonal/1983, 1984, 1987; Extrinsic/1984. Decreases in mean scores across all three time points were demonstrated by the following (scale/panel group): Intrinsic/1984, 1986, 1987, 1991; Involvement/1984, 1986, 1991; Interpersonal/1985, 1986, 1990, 1991; Extrinsic/1983, $1985,1986,1989,1991$. Consistent increases in subjects' mean scores for the three time points were reported on only the Intrinsic and Involvement subscales by the 1988 and 1983 groups respectively. Finally, a decrease in mean scores between the first and second time point, followed by an increase from the second to the third time points was demonstrated by the following (scale/panel group): Intrinsic/1985, 1989, 1990; Involvement/1985, 1988, 1989, 1990; Interpersonal/1988, 1989; Extrinsic/1987, 1988, 1990. 
In order to determine whether these changes in subjects' scores over time were statistically significant, RM-ANOVAs were conducted on the outcome variables of clinical competency, role adjustment, and job satisfaction. The first step in performing the RM-ANOVAs was to evaluate the data with regard to the test's statistical assumptions. The assumption of statistical independence of observations was met as each subject contributed scores to one, and only one, panel group. Inspection of the distributions of the dependent variables revealed some mild to moderate violations of normality. The assumption of equality of variances was also violated for all but three of the dependent variables (i.e., NCS total, JSI Involvement, JSI Extrinsic) as indicated in statistically significant tests of sphericity; however, the Greenhouse-Geisser values for the significant variables were large (.88 to .99), thus indicating that their departure from sphericity was minimal. Given the relative robustness of ANOVA models to violations of the assumptions of normality and homogeneity of variances, the violations existing in this data set were not considered to be deterrents to continuing with the RM-ANOVAs.

The results of the RM-ANOVAs are summarized in Table 7. New graduates' clinical competencies increased significantly over time for the NCA total $\left(F_{T}=72.26\right.$, $p<.0001)$ and each of the seven NCA subscales: Assessment $\left(F_{T}=46.07, p<.0001\right)$; Intervention $\left(F_{T}=81.75, p<.0001\right)$; Medications $\left(F_{T}=59.34, p<.0001\right)$; Documentation $\left(F_{T}=65.97, p<.0001\right)$; Risk Management $\left(F_{T}=32.81, p<.0001\right)$; Patient Support $\left(F_{T}=28.75, p<.0001\right)$; Professionalization $\left(F_{T}=45.75, p<.0001\right)$. In addition, subjects demonstrated significant increases across time in the aspect of their role functioning 
Table 7

RM-ANOVAs on the Outcome Variables for the Panel Year Groups

\begin{tabular}{lcccccc}
\hline \multicolumn{1}{c}{ Variable } & \multicolumn{2}{c}{ Panel Year Cohort } & \multicolumn{4}{c}{ Time } \\
& $F$ & $d f$ & $p<$ & $F$ & $d f$ & $p<$ \\
\hline NCA Total & 1.77 & 7,172 & --- & 72.26 & 2,344 & .0001 \\
Assessment & 2.19 & 7,179 & .037 & 46.07 & 2,358 & .0001 \\
Intervention & .71 & 7,221 & --- & 81.75 & 2,442 & .0001 \\
Medications & .96 & 7,220 & --- & 59.34 & 2,440 & .0001 \\
Documentation & .81 & 7,220 & --- & 65.97 & 2,440 & .0001 \\
Risk Management & .68 & 7,218 & --- & 32.81 & 2,436 & .0001 \\
Patient Support & .58 & 7,220 & --- & 28.75 & 2,440 & .0001 \\
Professionalization & 1.42 & 7,219 & --- & 45.75 & 2,438 & .0001 \\
NCS Total & 3.26 & 7,216 & .003 & 8.95 & 2,432 & .0001 \\
$R F S$ Total & 1.18 & 7,210 & --- & 34.41 & 2,420 & .0001 \\
JSI Total & 1.09 & 7,169 & --- & 1.89 & 2,338 & --- \\
$\quad$ Intrinsic & 1.10 & 7,175 & --- & 2.29 & 2,350 & -- \\
Involvement & .96 & 7,181 & --- & 3.06 & 2,362 & .048 \\
Interpersonal & 1.05 & 7,183 & --- & 2.04 & 2,366 & --- \\
Extrinsic & 2.14 & 7,176 & .041 & .14 & 2,352 & $\ldots$ \\
\hline
\end{tabular}

concerning confidence in their familiarity with work demands and the hospital environment (RFS total: $F_{T}=34.41, p<.0001$ ), and their level of job satisfaction with regard to involvement $\left(F_{T}=3.06, p<.048\right)$. However, statistically significant decreases were found on new graduates' NCS measure of role functioning which pertains to perceptions of actual professional nursing practice $\left(F_{T}=8.95, p<.0001\right)$. No significant changes over time were detected for subjects' scores on the JSI total $\left(F_{T}=1.89\right.$, n.s. $)$, nor 
for the JSI subscales regarding Intrinsic $\left(F_{T}=2.29\right.$, n.s. $)$, Interpersonal $\left(F_{T}=2.04\right.$, n.s. $)$, and Extrinsic $\left(F_{T}=.14\right.$, n.s. $)$.

Table 7 also summarizes the between-group (panel year) differences across the three time points on the outcome variables. As shown in the table, there were no significant differences between the nine panel groups on the NCA total, six of the NCA subscales, RFS total, JSI total, and three of the JSI subscales. However, significant differences between the groups existed on the NCA subscale for Assessment, the NCS total, and the Extrinsic subscale of the JSI. Post hoc tests revealed that the significant results between the groups on the Assessment subscale were due to differences between the 1984 and 1987 groups, on the one hand, and the 1991 group, on the other hand $\left(X^{2}=14.42, p<.04\right)$. Significant results between the groups on the NCS total were due to differences between the groups at each of the three time points: at $t_{1}\left(X^{2}=33.98\right.$, $p<.0001$ ), the 1983 group scored substantially lower than the $1985,1986,1987,1990$, and 1991 groups; at $t_{2}\left(X^{2}=17.02, p<.03\right)$, the 1984 subjects' scores were significantly lower than the 1991 subjects' scores; and at $t_{3}\left(X^{2}=16.81, p<.02\right)$, the mean scores for the 1984 and 1986 groups were significantly lower than those of the 1990 group. With regard to the JSI Extrinsic subscale, significant group differences occurred between the 1983 group and the 1985 group at $t_{1}\left(X_{2}=38.02, p<.0001\right)$, and the 1983 group and the 1990 group at $t_{3}\left(X_{2}=25.26, p<.001\right)$.

One-sample RM-ANOVAs were also conducted for each panel group separately to provide within-subjects measures on the outcome variables across the three time points. Table 8 summarizes these results, with the exception of two groups $(1988,1991)$ 
Table 8

One-Sample RM-ANOVAs for Each Panel Group on the Outcome Variables

\begin{tabular}{|c|c|c|c|c|c|c|c|c|c|c|c|c|c|c|c|c|c|c|c|c|c|c|c|c|c|}
\hline Variablo & & 1903 & & & 1984 & & & 1985 & & & 1966 & & & 1907 & & & 19 & & 1969 & & & 1990 & & & $1991^{*}$ \\
\hline & $\boldsymbol{F}$ & $\phi$ & $p<$ & $\boldsymbol{F}$ & $\phi$ & $p<$ & $\boldsymbol{F}$ & $\phi$ & $p<$ & $\boldsymbol{F}$ & $\phi$ & $p<$ & $\boldsymbol{F}$ & $\Phi$ & $p<$ & $\boldsymbol{F}$ & $\notin p<$ & $\boldsymbol{F}$ & $\Phi$ & $p<$ & $\boldsymbol{F}$ & $\$ 1$ & $p<$ & $\boldsymbol{F}$ & $\$ p<$ \\
\hline NCI Toend & 25.02 & 2,76 & .0001 & 43.73 & 2,62 & .0001 & 18.40 & 2,76 & .0001 & 66.41 & $2, x$ & .0001 & 17.96 & 2,16 & .0001 & & $N / A$ & 24.48 & 2,16 & .0001 & 21.93 & 2,28 . & .0001 & & $N / A$ \\
\hline 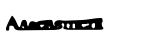 & 24.22 & 2,82 & .0001 & 37.88 & 2,64 & .0001 & 17.26 & 2,80 & .0001 & 47.98 & 2,70 & .0001 & 4.83 & 2,16 & .023 & & N/A & 18.21 & 2,16 & .0001 & 13.58 & 2,30 & .0001 & & N/A \\
\hline bneorvention & 34.58 & 2,84 & .0001 & 35.93 & 2,66 & .0001 & 27.54 & 2,90 & .0001 & 71.24 & 2,86 & .0001 & 33.84 & 2,38 & .0001 & & N/A & 14.12 & 2,32 & .0001 & 22.25 & $2,44$. & .0001 & & $N / A$ \\
\hline Modicatione & 37.26 & 2,82 & .0001 & 31.78 & 2,66 & .0001 & 13.60 & 2,90 & .0001 & 31.93 & 2,86 & .0001 & 15.07 & 2,38 & .0001 & & N/A & 15.22 & 2,32 & .0001 & 41.03 & 2,44 & .0001 & & N/A \\
\hline Dunmanetion & 25.55 & 2,84 & .0001 & 30.32 & 2,66 & .0001 & 17.93 & 2,88 & .0001 & 40.55 & 2,86 & .0001 & 15.11 & 2,38 & .0001 & & $N / A$ & 12.92 & 2,32 & .0001 & 28.01 & 2,44 & .0001 & & N/A \\
\hline Riol Momad & 12.71 & 2,82 & .0001 & 7.43 & 2,64 & .001 & 12.50 & 2,90 & .0001 & 17.22 & 2,86 & .0001 & 8.612 & 2,40 & .001 & & $N / A$ & 10.44 & 2,32 & .0001 & 10.54 & 2,40 & .0001 & & N/A \\
\hline Pi Support & 4.52 & 2,84 & .014 & 6.88 & 2,66 & .002 & 4.142 & 2,90 & .019 & 18.20 & 2,86 & .0001 & 11.33 & 2,38 & .0001 & & $N / A$ & 4.09 & 2,32 & .026 & 10.13 & 2,42 . & .0001 & & N/A \\
\hline Proficenionel & 18.09 & 2,82 & .0001 & 24.06 & 2,66 & .0001 & 18.71 & 2,88 & .0001 & 38.10 & 2,86 & .0001 & 9.82 & 2,40 & .0001 & & $N / A$ & 6.51 & 2,32 & .004 & 3.84 & 2,42 & .029 & & $N / A$ \\
\hline NCS Toued & 2.57 & 2,82 & - & 5.70 & 2,66 & .005 & 15.76 & 2,88 & .0001 & 13.64 & 2,84 & .0001 & 1.312 & 2,38 & - & & N/A & 4.14 & 2,32 & .025 & 5.26 & 2,40 & .009 & & $N / A$ \\
\hline RFS Toond & 30.86 & 2,78 & .0001 & 15.59 & 2,66 & .0001 & 23.95 & 2,88 & .0001 & 33.53 & 2,80 & .0001 & 12.56 & 2,36 & .0001 & & $N / A$ & 7.83 & 2,32 & .002 & $17 . \pi$ & 2,40 . & .0001 & & $N / A$ \\
\hline JSI Toad & .23 & 2,68 & - & .32 & 2,52 & - & .22 & 2,76 & - & 1.04 & 2,64 & - & .492 & 220 & - & & N/A & .48 & 2,22 & - & 2.75 & 2,36 & - & & $N / A$ \\
\hline merimeic & .36 & 2,70 & - & .332 & 2,54 & - & .932 & 2,76 & - & 2.61 & 2,66 & - & .562 & 2,22 & - & & N/A & 3.47 & 2,22 & .049 & 2.352 & 2,40 & - & & N/A \\
\hline Enotraned & 2.93 & 2,74 & - & .10 & 2,56 & - & 1.99 & 2,76 & - & .08 & 2,70 & - & .452 & 2,22 & - & & $N / A$ & .76 & 2,24 & - & .472 & 2,40 & - & & N/A \\
\hline brespersonal & 2.07 & 2,74 & - & 1.35 & 2,58 & - & 2.212 & 2,76 & - & 1.61 & 2,70 & - & .812 & 2,22 & - & & N/A & .27 & 2,24 & - & 2.94 & 2,42 & - & & N/A \\
\hline Extrumatic & 10.90 & 2,70 & - & .332 & 2,58 & - & 3.31 & 2,76 & .042 & 1.73 & 2,68 & - & 2.082 & 2,20 & - & & $N / A$ & 3.17 & 2,22 & - & 2.432 & 2,38 & - & & N/A \\
\hline
\end{tabular}

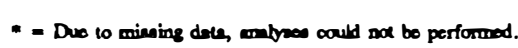


for which the analyses could not be performed due to missing data. This table illustrates that each of the panel year groups demonstrated significant increases in mean scores for the NCA total, the seven NCA subscales, and the RFS total. On the NCS, subjects in five of the groups $(1984,1985,1986,1989,1990)$ had significant decreases in their scores, while the other two groups' changes in mean scores were nonsignificant. With regard to job satisfaction, each panel group's change in mean scores was nonsignificant on the JSI total, and the Involvement and Interpersonal subscales. However, on the Intrinsic subscale, a significant decrease was detected for the 1989 group; and on the Extrinsic subscale, the 1983 and 1985 panel groups demonstrated statistically significant decreases in subjects' mean scores.

Research Question 2: Is the length of stay of neophyte nurses participating in the transition program longer than the length of stay of new graduate nurses employed prior to implementation of the transition program? The length of stay (LOS) for new graduates in each panel year and the aggregate sample are provided in Table 9. The table demonstrates that the aggregate sample's average LOS is slightly higher $(M=33.16$, $S D=30.84)$ than the average LOS of new graduate nurses at the hospital study site prior to implementation of the transition program, which was 30 months. It should be noted that, as evidenced in Table 9, the average LOS shows some variation for the years 1983 through 1988 but for the remaining three years the values consistently decline. These declining values are more an artifact of the timing of data collection rather than true reflections of length of stay. In other words, because the secondary data set with respect 
Table 9

Length of Stay for Each Panel Year and the Aggregate Sample

\begin{tabular}{|c|c|c|c|c|c|c|c|c|c|c|}
\hline Variable & 1983 & 1984 & 1985 & 1986 & 1987 & 1988 & 1989 & 1990 & 1991 & Aggregate \\
\hline \multicolumn{11}{|l|}{ LOS (yrs) } \\
\hline Range & $0-9.5$ & $0-8.6$ & $0-7.9$ & $0-6.9$ & $0-5.8$ & $0-4.8$ & $0-3.7$ & $0-2.6$ & $0-1.9$ & $0-9.5$ \\
\hline$M$ & 4.19 & 3.31 & 3.05 & 3.01 & 2.35 & 2.86 & 2.16 & 1.94 & 1.31 & 2.78 \\
\hline$m d n$ & 3.00 & 2.30 & 2.05 & 2.35 & 1.30 & 3.40 & 3.00 & 2.50 & 1.50 & 2.00 \\
\hline$S D$ & 3.55 & 3.03 & 2.94 & 2.63 & 2.18 & 1.68 & 1.48 & .87 & .43 & 2.57 \\
\hline \multicolumn{11}{|l|}{ LOS (mos) } \\
\hline Range & $0-115$ & $0-103$ & $0-95$ & $0-83$ & $0-69$ & $0-58$ & $0-45$ & $0-31$ & $0-23$ & $0-115$ \\
\hline$M$ & 50.29 & 39.10 & 36.55 & 36.14 & 27.57 & 34.31 & 25.69 & 22.87 & 15.78 & 33.16 \\
\hline$m d n$ & 36.00 & 27.50 & 24.50 & 28.00 & 14.00 & 41.00 & 36.00 & 29.00 & 18.00 & 23.00 \\
\hline$S D$ & 42.64 & 36.32 & 35.28 & 31.49 & 26.23 & 20.17 & 17.56 & 10.19 & 5.25 & 30.84 \\
\hline
\end{tabular}


to LOS was updated as of December 1992, the last three panel years do not have the same opportunity to demonstrate as long a LOS as the preceding years. Thus, the lowermean LOS of the 1989, 1990, and 1991 groups skew the mean LOS for the aggregate sample. In an attempt to correct for this problem, the LOS values for these three groups were treated as outliers, and a revised average LOS was calculated for a sub-aggregate sample which included only the years 1983 through 1988 . The revised sub-aggregate mean LOS of 37.5 months was also higher than the 30 -month average LOS reported for neophyte nurses prior to implementation of the transition program.

Research Question 3: Is the turnover rate of neophyte nurses participating in the transition program less than the turnover rate of new graduate nurses employed prior to implementation of the transition program? Table 10 provides the yearly turnover rates (TOR) for the first three years for each panel year and the aggregate sample. The TOR during the first year ranged from $15.3 \%$ to $42.9 \%$ with a TOR of $27.8 \%$ for the total sample. During the second year of employment, the TOR decreased for the aggregate sample to $14.2 \%$, with panel group ranges from $10.9 \%$ to $20.6 \%$. During the third year on the job, up to 30 months, an average of 6.2 percent of the new graduates had resigned. In comparison to a $48 \%$ pre-program neophyte TOR, the data provided in Table 10 reveal a substantially lower rate of attrition for new graduates after implementation of the transition program. None of the panel groups, nor the aggregate sample, demonstrated a TOR of greater than $42.9 \%$ (1987 group), even at the time of highest tumover which was during the first year of employment. 
Table 10

Tumover Rates (TOR) for Each Panel Year and the Aggregate Sample

\begin{tabular}{||c|c|c|c|c|c|c|c|c|c|c|}
\hline Variable & 1983 & 1984 & 1985 & 1986 & 1987 & 1988 & 1989 & 1990 & 1991 & Aggregate \\
\hline TOR: & $f(\%)$ & $f(\%)$ & $f(\%)$ & $f(\%)$ & $f(\%)$ & $f(\%)$ & $f(\%)$ & $f(\%)$ & $f(\%)$ & $f(\%)$ \\
\hline$<1$ year & $34(21.9)$ & $20(29.9)$ & $40(36.4)$ & $39(34.2)$ & $39(42.9)$ & $13(19.1)$ & $30(32.6)$ & $16(17.6)$ & $15(15.3)$ & $246(27.8)$ \\
\hline$<2$ years & $32(20.6)$ & $7(10.4)$ & $14(12.7)$ & $14(12.3)$ & $11(12.1)$ & $12(17.6)$ & $10(10.9)$ & $12(13.2)$ & - & $112(14.2)$ \\
\hline$<2.5$ yrs & $9(5.8)$ & $8(11.9)$ & $9(8.2)$ & $7(6.1)$ & $6(6.6)$ & $2(2.9)$ & $2(2.2)$ & - & - & $43(6.2)$ \\
\hline
\end{tabular}


Research Question 4: Are there significant differences with regard to clinical competency, role adjustment, and job satisfaction between neophyte nurses whose length of stay exceeds 15 months (stayers) and neophyte nurses who resign within 15 months of employment (leavers)? Descriptive summary statistics for the two groups designated as stayers $(n=583)$ and leavers $(n=306)$ on the outcome variables at each of the three time points are provided in Table 11. As the table illustrates, stayers and leavers each entered the transition program with a moderate level of proficiency with Table 11

Measures of Central Tendency and Dispersion on the Outcome Variables for. Stayers and Leavers at Each Time Point

\begin{tabular}{|c|c|c|c|c|}
\hline \multirow[t]{2}{*}{ Variable } & \multicolumn{2}{|c|}{$\begin{array}{c}\text { Stayers } \\
(n=583)\end{array}$} & \multicolumn{2}{|c|}{$\begin{array}{l}\text { Leavers } \\
(n=306)\end{array}$} \\
\hline & $M$ & $S D$ & $M$ & $S D$ \\
\hline \multicolumn{5}{|l|}{ NCA Total } \\
\hline$t_{1}$ & 108.74 & 16.73 & 109.67 & 14.64 \\
\hline$t_{2}$ & 122.65 & 13.89 & 122.45 & 13.09 \\
\hline$t_{3}$ & 128.36 & 13.95 & 128.02 & 11.90 \\
\hline \multicolumn{5}{|l|}{ Assessment } \\
\hline$t_{1}$ & 24.05 & 4.10 & 23.94 & 3.76 \\
\hline$t_{2}$ & 27.39 & 4.09 & 27.38 & 3.66 \\
\hline$t_{3}$ & 29.20 & 3.64 & 28.39 & 3.34 \\
\hline \multicolumn{5}{|l|}{ Intervention } \\
\hline$t_{1}$ & 13.94 & 2.69 & 13.74 & 2.64 \\
\hline$t_{2}$ & 16.39 & 2.32 & 16.35 & 1.97 \\
\hline$t_{3}$ & 17.22 & 2.16 & 17.27 & 1.90 \\
\hline \multicolumn{5}{|l|}{ Medications } \\
\hline$t_{1}$ & 7.00 & 1.47 & 6.97 & 1.52 \\
\hline$t_{2}$ & 8.01 & 1.38 & 8.11 & 1.15 \\
\hline$t_{3}$ & 8.50 & 1.22 & 8.59 & 1.09 \\
\hline \multicolumn{5}{|l|}{ Documentation } \\
\hline$t_{1}$ & 10.89 & 2.21 & 10.86 & 2.09 \\
\hline$t_{2}$ & 12.47 & 1.83 & 12.62 & 1.81 \\
\hline$t_{3}$ & 13.16 & 1.68 & 13.48 & 1.48 \\
\hline \multicolumn{5}{|l|}{ Risk Management } \\
\hline$t_{1}$ & 11.83 & 2.24 & 11.88 & 2.03 \\
\hline$t_{2}$ & 12.92 & 1.81 & 13.11 & 1.68 \\
\hline$t_{3}$ & 13.50 & 1.53 & 13.38 & 1.52 \\
\hline
\end{tabular}




\begin{tabular}{|c|c|c|c|c|}
\hline Variable & $M$ & $S D$ & $M$ & $S D$ \\
\hline \multicolumn{5}{|l|}{ Patient Support } \\
\hline$t_{1}$ & 20.08 & 3.53 & 20.32 & 3.25 \\
\hline$t_{2}$ & 21.39 & 3.10 & 21.29 & 2.98 \\
\hline$t_{3}$ & 22.21 & 2.60 & 21.86 & 2.37 \\
\hline \multicolumn{5}{|l|}{ Professionalization } \\
\hline$t_{1}$ & 21.33 & 4.05 & 21.76 & 3.57 \\
\hline$t_{2}$ & 23.31 & 3.28 & 23.30 & 3.10 \\
\hline$t_{3}$ & 24.56 & 3.39 & 24.83 & 2.75 \\
\hline \multicolumn{5}{|l|}{ NCS Total } \\
\hline$t_{1}$ & 51.68 & 6.35 & 51.74 & 5.88 \\
\hline$t_{2}$ & 49.47 & 6.88 & 49.10 & 6.11 \\
\hline$t_{3}$ & 48.83 & 7.16 & 49.29 & 6.94 \\
\hline \multicolumn{5}{|l|}{ RFS Total } \\
\hline$t_{1}$ & 72.46 & 11.28 & 72.78 & 10.41 \\
\hline$t_{2}$ & 78.81 & 10.24 & 78.90 & 8.47 \\
\hline$t_{3}$ & 83.74 & 9.75 & 83.87 & 8.77 \\
\hline \multicolumn{5}{|l|}{ JSI Total } \\
\hline$t_{1}$ & 61.09 & 9.06 & 61.02 & 8.72 \\
\hline$t_{2}$ & 60.34 & 8.78 & 59.62 & 7.50 \\
\hline$t_{3}$ & 59.28 & 8.37 & 59.29 & 8.33 \\
\hline \multicolumn{5}{|l|}{ Intrinsic } \\
\hline$t_{1}$ & 15.77 & 2.60 & 15.78 & 2.70 \\
\hline$t_{2}$ & 15.55 & 2.61 & 15.54 & 2.18 \\
\hline$t_{3}$ & 15.44 & 2.51 & 15.21 & 2.52 \\
\hline \multicolumn{5}{|l|}{ Involvement } \\
\hline$t_{1}$ & 14.70 & 2.68 & 14.79 & 2.55 \\
\hline$t_{2}$ & 14.94 & 2.64 & 14.63 & 2.43 \\
\hline$t_{3}$ & 14.95 & 2.44 & 15.06 & 2.31 \\
\hline \multicolumn{5}{|l|}{ Interpersonal } \\
\hline$t_{1}$ & 16.44 & 2.67 & 16.24 & 2.61 \\
\hline$t_{2}$ & 16.15 & 2.48 & 15.89 & 2.28 \\
\hline$t_{3}$ & 15.75 & 2.55 & 15.62 & 2.61 \\
\hline \multicolumn{5}{|l|}{ Extrinsic } \\
\hline$t_{1}$ & 14.29 & 2.73 & 14.26 & 2.53 \\
\hline$t_{2}$ & 13.69 & 2.73 & 13.59 & 2.50 \\
\hline$t_{3}$ & 13.12 & 2.71 & 13.39 & 2.41 \\
\hline
\end{tabular}

regard to their overall clinical competency (NCA) and their specific competencies (NCA subscales). In addition, both groups demonstrated an increase in mean scores on the NCA total and the NCA subscales across all three time points with mean scores at $t_{3}$ indicating a moderately high level of clinical proficiency. 
With regard to role adjustment, the new graduates in each group displayed a moderately positive perception of nursing practice and a moderately high degree of confidence in their familiarity with work demands and the hospital environment both upon employment and after one year on the job. Specifically, on the NCS, while stayers' mean scores declined slightly from $t_{1}$ to $t_{3}$, leavers' scores also decreased from the first to the second time point, but increased at the third time point. And on the RFS, both groups' mean scores increased across the time points.

Job satisfaction scores (JSI) for subjects in the two groups indicated a moderately high degree of overall satisfaction across the groups upon hire and one year later despite a decrease in mean scores on the JSI total across the three time points. In addition, both groups' scores declined on the Intrinsic, Interpersonal, and Extrinsic subscales. In contrast, stayers and leavers demonstrated increases in mean scores on the Involvement subscale, although leavers did demonstrate a decline in their scores from $t_{1}$ to $t_{2}$.

Prior to determining whether there were significant differences between stayers and leavers on the outcome variables, the data were evaluated with regard to the statistical assumptions for RM-ANOVAs. The assumption of statistical independence of observations was met as each subject contributed scores to one, and only one, group. Evaluation of the distributions of the dependent variables revealed some mild to moderate violations of normality. The assumption of equality of variances was also violated for all but three of the dependent variables as indicated in statistically significant tests of sphericity. However, the Greenhouse-Geisser values of the significant variables were large (.88 to 1.00$)$, indicating that the departure from sphericity was minimal. 
Table 12 summarizes the results of the RM-ANOVAs. There were no significant differences between stayers and leavers with regard to their mean scores on the outcome Table 12

RM-ANOVAs on the Outcome Variables for Stayers and Leavers

\begin{tabular}{lcccccc}
\hline \multicolumn{1}{c}{ Variable } & \multicolumn{5}{c}{ Groups } & \multicolumn{3}{c}{ Time } \\
& $F$ & $d f$ & $p<$ & $F$ & $d f$ & $p<$ \\
\hline NCA Total & .11 & 1,178 & - & 123.89 & 2,356 & .0001 \\
Assessment & .40 & 1,185 & - & 95.11 & 2,370 & .0001 \\
Intervention & 1.20 & 1,227 & - & 156.31 & 2,454 & .0001 \\
Medications & .87 & 1,226 & - & 127.60 & 2,452 & .0001 \\
Documentation & .00 & 1,226 & - & 118.54 & 2,452 & .0001 \\
Risk Management & .26 & 1,224 & - & 55.38 & 2,448 & .0001 \\
Patient Support & 1.47 & 1,226 & - & 31.07 & 2,452 & .0001 \\
Professionalization & .01 & 1,225 & -- & 77.60 & 2,450 & .0001 \\
NCS Total & .15 & 1,222 & - & 25.35 & 2,444 & .0001 \\
RFS Total & .96 & 1,216 & - & 102.41 & 2,432 & .0001 \\
JSI Total & .03 & 1,175 & - & 2.52 & 2,350 & - \\
Intrinsic & .08 & 1,181 & - & 1.56 & 2,362 & - \\
Involvement & .11 & 1,187 & - & .36 & 2,374 & - \\
Interpersonal & .21 & 1,189 & -- & 2.96 & 2,378 & - \\
Extrinsic & .10 & 1,182 & - & 9.02 & 2,364 & .0001 \\
\hline
\end{tabular}

variables measuring clinical competency (NCA Total: $\left.F_{G}=.11, n . s.\right)$, role adjustment (NCS: $F_{G}=.15$, n.s.; RFS: $F_{G}=.96$, n.s.), or job satisfaction (JSI Total: $F_{G}=.03$, n.s.). Although stayers' had higher mean scores on more variables at $t_{1}$ and $t_{2}$ than leavers, and leavers had twice as many higher mean scores than stayers at $t_{3}$, these differences between the groups were not significant. 
As Table 12 also illustrates, stayers and leavers did demonstrate significant increases in their mean scores over time on the NCA total $\left(F_{T}=123.89, p<.0001\right)$, and each of the seven NCA subscales: Assessment $\left(F_{T}=95.11, p<.0001\right)$; Intervention $\left(F_{T}=156.31, \quad p<.0001\right) ; \quad$ Medications $\quad\left(F_{T}=127.60, \quad p<.0001\right) ; \quad$ Documentation $\left(F_{T}=118.54, p<.0001\right) ;$ Risk Management $\left(F_{T}=55.38, p<.0001\right)$; Patient Support $\left(F_{T}=31.07, p<.0001\right)$; Professionalization $\left(F_{T}=77.60, p<.0001\right)$. In addition, subjects in these groups demonstrated significant increases over time on the RFS variables measuring role adjustment $\left(F_{T}=102.41, p<.0001\right)$, while also displying a significant decrease over time on the NCS aspect of role adjustment $\left(F_{T}=25.35, p<.0001\right)$. Although there were no significant changes for time in the groups' scores for the JSI total $\left(F_{T}=2.52, n . s.\right)$ and three of the JSI subscales (Intrinsic: $F_{T}=1.56$, n.s.; Involvement: $F_{T}=.36$, n.s.; Interpersonal: $F_{T}=2.96$, n.s. $)$, a significant decrease was detected in the subscale measuring extrinsic job satisfaction $\left(F_{T}=9.02, p<.0001\right)$.

Research Question 5: What professional and role-oriented values do neophyte nurses possess upon initial employment? In order to identify new graduates' professional and role-oriented values, two separate factor analyses (FA) were performed using subjects' scores obtained at $\mathrm{t}_{1}$ on the Nursing Characteristics Scale (NCS) and the Role Function Survey (RFS). Prior to conducting the FAs, the data were evaluated for according to the criteria described in Chapter 3. The observation-to-variable ratios were 36:1 for the NCS and 35:1 for the RFS, which is sufficient to generate statistically stable factor structures. The suitability of the correlation matrix for each scale was examined to determine the appropriateness of the factor models. All indices were well within 
acceptable ranges. The Kaiser-Meyer-Olkin (KMO) indexes were .889 and .872 for the NCS and the RFS respectively. The Bartlett sphericity $X^{2}$ indexes of 3310.89 $(p<.000001)$ for the NCS and $5268.69(p<.000001)$ for the RFS indicated that neither correlation matrix was an identity matrix and thus both were suitable for factor analysis. Lastly, with respect to the NCS, a total of 36 or $19.8 \%$ of the off-diagonals in the antiimage covariance matrix (AIC) were $>.09$, indicating that $70.2 \%$ of the partial correlation coefficients were $>.09$. And, on the RFS, a total of $28(10.3 \%)$ of the offdiagonals in the AIC were $>.09$, signifying that $79.7 \%$ of the partial correlation coefficients were $>.09$. These values show that there were sufficient association among each instrument's items to extract possible factor structures and to identify differentiated factor structures.

NCS Factor Analysis. Using the criteria of a minimum eigenvalue of 1.0, initial extraction of the NCS items yielded a three-factor solution. However, because the mean communality was inadequate (.54), the Cattall scree test was also utilized. Visual inspection of the scree plot confirmed the retention of three factors, which were then subjected to varimax rotation. All instrument items were sorted into factors using the minimum loading of .32 as the cutoff, which resulted in factor loadings of .51 or more for each factor. No ambiguous or dual loadings were observed. Table 13 summarizes the results of the three factor solution which accounted for $54 \%$ of the total variance. The dimensions reflected in the factor structure were named as follows. Factor I, Personal and Professional Intrinsic Rewards, was composed of nine variables which 
Table 13

NCS Factor Structure

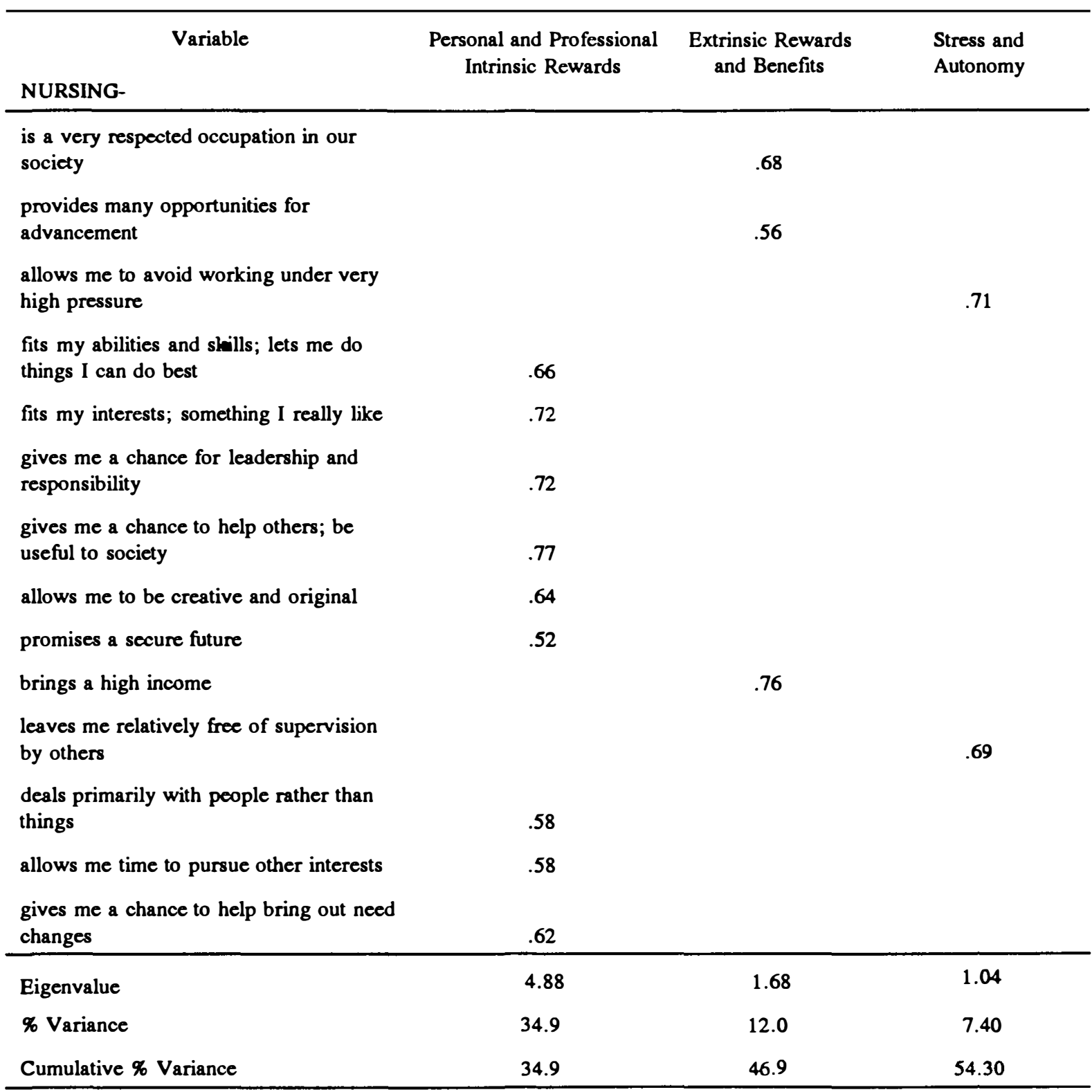

accounted for almost $35 \%$ of the variance of the three-factor solution. These items reflected values associated with both personal and professional benevolence, growth,opportunity, and long-term employment security. Factor 2, Extrinsic Rewards 
and Benefits, representing $12 \%$ of the solution's variance, was comprised of three variables that depicted values of societal and professional recognition, as well as financial security. Factor 3, Stress and Autonomy, consisted of two variables which addressed stress manifestation and professional independence. This factor accounted for the least amount of the solution's variance, or 7.4 percent.

RFS Factor Analysis. Initial extraction of the RFS produced 5 factors with a minimum eigenvalue of 1.0 and a mean communality $\left(h^{2}\right)$ of .63 . Although the mean $h^{2}$ was adequate, examination of the scree plot confirmed the appropriateness of retaining the five factors. This initial solution was rotated using the varimax approach and the instrument's items were sorted for minimum loadings of .32 per factor. Factor loadings ranged from .46 to .79 , and there were no ambiguous or dual loadings. Summarized in Table 14, this five-factor solution accounted for $63 \%$ of the total variance. The dimensions reflected in the factor structure were named in the following manner. Factor I, Organizational Relationships, accounted for $37 \%$ of the solution's variance and was composed of seven items. These items reflected values pertaining to communicating and working effectively with others and within the organizational system. Factor 2, Organizational Functioning, was comprised of four variables representing values associated with getting work accomplished, such as identifying and utilizing resources, policies and procedures. Ten percent of the solution's variance was explained by this factor. Factor 3, Work Group Responsibilities and Job Conditions, accounted for 6\% of the variance and consisted of five items which addressed unit work roles and adjustment 
Table 14

\section{RFS Factor Structure}

\begin{tabular}{|c|c|c|c|c|c|}
\hline Variable & $\begin{array}{l}\text { Organizational } \\
\text { Relationships }\end{array}$ & $\begin{array}{l}\text { Organizational } \\
\text { Functioning }\end{array}$ & $\begin{array}{c}\text { Work Group } \\
\text { Responsibilities \& } \\
\text { Job Conditions }\end{array}$ & $\begin{array}{l}\text { Professional } \\
\text { Development }\end{array}$ & Nursing Process \\
\hline
\end{tabular}

Utilize the nursing process in giving patient care

Accurately and consistently document care

Familiar with care protocols relating to clinical area

Familiar with unit policies and procedures

Familiar with hospital philosophy and objectives

Identify hospital resources both human and material which contribute to care delivery

Use hospital resources appropriately

Feel comfortable as part of unit work group

Adjust to time schedule and demands of staffing pattern

Set own personal goals for continued growth and professional development

Assess leaming needs and seek out educational opportunities 


\begin{tabular}{|c|c|c|c|c|c|}
\hline Variable & $\begin{array}{l}\text { Organizational } \\
\text { Relationships }\end{array}$ & $\begin{array}{l}\text { Organizational } \\
\text { Functioning }\end{array}$ & $\begin{array}{l}\text { Work Group } \\
\text { Responsibilities \& } \\
\text { Job Conditions }\end{array}$ & $\begin{array}{l}\text { Professional } \\
\text { Development }\end{array}$ & Nursing Process \\
\hline Understand charge nurse responsibilities & & & .46 & & \\
\hline $\begin{array}{l}\text { Supervise and direct ancillary care } \\
\text { personnel }\end{array}$ & & & 47 & & \\
\hline $\begin{array}{l}\text { Work with the organizational and } \\
\text { bureaucratic system }\end{array}$ & .56 & & & & \\
\hline $\begin{array}{l}\text { Cooperate knowledgeably with other } \\
\text { hospital services and departments }\end{array}$ & .59 & & & & \\
\hline Demonstrate good communications skills & .60 & & & & \\
\hline Deal assertively with conflict situations & .76 & & & & \\
\hline Relate collegially with the medical staff & .76 & & & & \\
\hline Cope constructively with work stress & .63 & & & & \\
\hline $\begin{array}{l}\text { Solve most work related problems as they } \\
\text { arise }\end{array}$ & .66 & & & & \\
\hline Feel satisfied with working conditions & & & .62 & & \\
\hline Eigenvalue & 7.83 & 2.12 & 1.26 & 1.05 & 1.02 \\
\hline \% Variance & 37.30 & 10.10 & 6.0 & 5.0 & 4.9 \\
\hline Cumulative $\%$ Variance & 37.30 & 47.40 & 53.4 & 58.4 & 63.3 \\
\hline
\end{tabular}


to time schedules and staffing. Factor 4, Professional Development, with $5 \%$ of the variance, consisted of two items and dealt with values regarding the setting ofprofessional goals, assessing learning needs, and seeking educational opportunities. Factor 5, Nursing Process, was comprised of three variables which addressed values pertaining to familiarity with clinical protocols and utilization of the nursing process. This last factor explained $4.9 \%$ of the solution's variance.

Research Question 6: Do neophytes nurses professional and role-oriented values change over time? In order to determine whether the subjects professional and roleoriented values changed over time, the factor scores retained from the NCS and RFS factor analyses were entered as the dependent variables in RM-ANOVAs. Table 15 displays the descriptive summary statistics for the three factors of the NCS and the five Table 15

Measures of Central Tendency and Dispersion for the NCS and. $\underline{\text { RFS Factors at the Three Time Points }}$

\begin{tabular}{|c|c|c|c|}
\hline Factor Name & $M \stackrel{t_{1}}{(S D)}$ & $\stackrel{t_{2}}{M}(S D)$ & $M \stackrel{t_{3}}{(S D)}$ \\
\hline \multicolumn{4}{|l|}{ NCS: } \\
\hline Personal and Professional Intrinsic Rewards & $6.19(.79)$ & $5.85(.81)$ & $5.86(.85)$ \\
\hline Extrinsic Rewards and Benefits & $4.75(.93)$ & $4.48(1.03)$ & $4.32(1.07)$ \\
\hline Stress and Autonomy & $1.97(.64)$ & $2.03(.65)$ & $1.87(.87)$ \\
\hline \multicolumn{4}{|l|}{$R F S:$} \\
\hline Organizational Relationships & $5.45(.93)$ & $5.69(.82)$ & $6.00(.81)$ \\
\hline Organizational Functioning & $3.62(.85)$ & $4.14(.66)$ & $4.38(.65)$ \\
\hline $\begin{array}{l}\text { Work Group Responsibilities and Job } \\
\text { Conditions }\end{array}$ & $4.57(1.01)$ & $5.09(.89)$ & $5.42(.85)$ \\
\hline Professional Development & $3.81(.64)$ & $3.73(.59)$ & $3.87(.59)$ \\
\hline Nursing Process & $4.42(.71)$ & $4.87(.64)$ & $5.19(.62)$ \\
\hline
\end{tabular}


factors of the RFS at each of the three time points. On the NCS, the assumptions for RM-ANOVA concerning statistical independence and equality of variance were met, but there were mild violations of the assumption of normality. For the RFS, the assumption of statistical independence was also met, but there were violations of the normality and homogeneity of variance assumptions. However, Greenhouse-Geisser values ranging from .84 to .94 indicated only a minimal departure from sphericity.

Results of the RM-ANOVAs on the NCS and RFS factors are provided in Table 16. The findings indicated that new graduate nurses' values decreased significantly over time on each of the three NCS factors, that is: personal and professional intrinsic rewards $\left(F_{T}=9.88, p<.0001\right)$; extrinsic rewards and benefits $\left(F_{T}=8.55, p<.0001\right)$; and stress and autonomy $\left(F_{T}=3.49, p<.031\right)$. In contrast, there was a significant increase Table 16

\section{RM-ANOVAs on the NCS and RFS Factors}

\begin{tabular}{lccc}
\hline \multicolumn{1}{c}{ Factor Name } & $F$ & $d f$ & $p<$ \\
\hline$N C S:$ & & & \\
Personal and Professional Intrinsic Rewards & 9.88 & 2,440 & .0001 \\
Extrinsic Rewards and Benefits & 8.55 & 2,438 & .0001 \\
Stress and Autonomy & 3.49 & 2,438 & .031 \\
$R F S:$ & & & \\
Organizational Relationships & 34.55 & 2,440 & .0001 \\
Organizational Functioning & 61.74 & 2,444 & .0001 \\
Work Group Responsibilities and Job Conditions & 18.77 & 2,426 & .0001 \\
Professional Development & 5.81 & 2,444 & .003 \\
Nursing Process & 77.12 & 2,444 & .0001 \\
\hline
\end{tabular}


for time on each of the five RFS factors: organizational relationships $\left(F_{T}=34.35\right.$, $p<.0001)$; organizational functioning $\left(F_{T}=61.74, p<.0001\right)$; work group responsibilities and job conditions $\left(F_{T}=18.77, p<.0001\right)$; professional development $\left(F_{T}=5.81, p<.003\right)$; and nursing process $\left(F_{T}=77.12, p<.0001\right)$.

Research Question 7: Are there significant differences with regard to professional and role-oriented values between neophyte nurses whose length of stay exceeds 15 months (stayers) and neophyte nurses who resign within 15 months of employment (leavers)? Table 17 displays the descriptive summary statistics on the three factors of the NCS and the five factors of the RFS for the two groups at the three time points.

\section{Table 17}

Measures of Central Tendency and Dispersion on the NCS and RFS Factors for Stayers and Leavers at the Three Time Points

\begin{tabular}{|c|c|c|}
\hline Factor Name & $\begin{array}{l}\text { Stayers } \\
M(S D)\end{array}$ & $\begin{array}{l}\text { Leavers } \\
M(S D)\end{array}$ \\
\hline \multicolumn{3}{|l|}{ NCS: } \\
\hline \multicolumn{3}{|l|}{ Personal and Professional Intrinsic Rewards } \\
\hline$t_{1}$ & $6.19(.82)$ & $6.19(.74)$ \\
\hline$t_{2}$ & $5.86(.85)$ & $5.81(.71)$ \\
\hline 5 & $5.85(.87)$ & $5.91(.76)$ \\
\hline \multicolumn{3}{|l|}{ Extrinsic Rewands and Benefits } \\
\hline$t_{1}$ & $4.71(.95)$ & $4.80(.89)$ \\
\hline$t_{2}$ & $4.50(1.02)$ & $4.46(1.06)$ \\
\hline$t_{3}$ & $4.32(1.05)$ & $4.35(1.17)$ \\
\hline \multicolumn{3}{|l|}{ Stress and Autonomy } \\
\hline 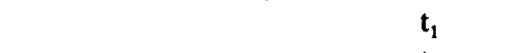 & $1.98(.64)$ & $1.95(.63)$ \\
\hline$t_{2}$ & $2.05(.65)$ & $1.96(.63)$ \\
\hline$t_{3}$ & $1.95(.67)$ & 2.07 (.59) \\
\hline \multicolumn{3}{|l|}{ RFS: } \\
\hline \multicolumn{3}{|l|}{ Organizational Relationships } \\
\hline$t_{1}$ & $5.45(.96)$ & $5.44(.89)$ \\
\hline$t_{2}$ & $5.68(.85)$ & $5.75(.73)$ \\
\hline$t_{3}$ & $5.99(.84)$ & $6.04(.69)$ \\
\hline
\end{tabular}




\begin{tabular}{|c|c|c|}
\hline Factor Name & $\begin{array}{l}\text { Stayers } \\
M(S D)\end{array}$ & $\begin{array}{l}\text { Leavers } \\
M(S D)\end{array}$ \\
\hline \multicolumn{3}{|l|}{ Organizational Functioning } \\
\hline 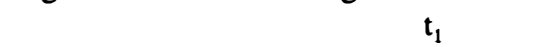 & $3.63(.85)$ & $3.60(.84)$ \\
\hline$t_{2}$ & $4.13(.67)$ & $4.17(.64)$ \\
\hline$t_{3}$ & $4.38(.65)$ & $4.39(.63)$ \\
\hline \multicolumn{3}{|c|}{ Work Group Responsibilities and Job Conditions } \\
\hline$t_{1}$ & $4.54(1.04)$ & $4.64(.94)$ \\
\hline$t_{2}$ & $5.11(.90)$ & $5.03(.86)$ \\
\hline$t_{3}$ & $5.41(.86)$ & $5.47(.81)$ \\
\hline \multicolumn{3}{|l|}{ Professional Development } \\
\hline$t_{1}$ & $3.78(.66)$ & $3.87(.61)$ \\
\hline$t_{2}$ & $3.72(.59)$ & $3.76(.60)$ \\
\hline$t_{3}$ & $3.86(.60)$ & $3.91(.52)$ \\
\hline \multicolumn{3}{|l|}{ Nursing Process } \\
\hline$t_{1}$ & $4.45(.72)$ & $4.38(.70)$ \\
\hline$t_{2}$ & $4.86(.66)$ & $4.90(.60)$ \\
\hline$t_{3}$ & $5.23(.61)$ & $5.05(.66)$ \\
\hline
\end{tabular}

As the table indicates, the factor scores declined over time for both groups on all three of the NCS factors, while increasing also for both groups on the RFS factors. In addition, on each NCS and RFS factor, leavers demonstrated slightly higher factor scores than stayers.

In order to determine whether these differences in factor scores between stayers and leavers were significant, RM-ANOVAs were performed with each group's factor scores serving as the dependent variables. Prior to conducting the analyses, the data was evaluated with regard to meeting the assumptions of the test. On the NCS, the assumptions concerning independence and equality of variance were met, but there were mild violations of the assumption of normality. For the RFS, the assumption of independence was also met, but there were violations of the normality and homogeneity of variance assumptions. However, Greenhouse-Geisser values ranging from .84 to .94 indicated that this departure from sphericity was minimal. 
Table 18 shows the results of the RM-ANOVAs conducted on the NCS and the RFS factors for stayers and leavers. As the table shows, the differences between the two Table 18

RM-ANOVAs on the NCS and RFS Factors for Stayers and Leavers

\begin{tabular}{lccc}
\hline \multicolumn{1}{c}{ Factor Name } & $F$ & $d f$ & $p<$ \\
\hline$N C S:$ & & & \\
Personal and Professional Intrinsic Rewards & .01 & 2,226 & -- \\
Extrinsic Rewards and Benefits & .11 & 2,225 & -- \\
Stress and Autonomy & .99 & 2,225 & -- \\
$R F S:$ & & & \\
Organizational Relationships & .99 & 2,226 & -- \\
Organizational Functioning & .00 & 2,228 & -- \\
Work Group Responsibilities and Job Conditions & .02 & 2,219 & -- \\
Professional Development & .03 & 2,228 & -- \\
Nursing Process & 2.57 & 2,228 & -- \\
\hline
\end{tabular}

groups' factor scores were nonsignificant. Therefore, it was concluded that differences in subjects' professional and role-oriented values did not discriminate between stayers and leavers in this study.

\section{$\underline{\text { Summary }}$}

Results of the data collected at three time points on new graduate staff nurses who participated in a neophyte transition program were presented in this chapter. Selected instruments measured subjects' clinical competency, role adjustment, and job satisfaction. In addition, differences between stayers and leavers with respect to the outcome variables were examined, and subjects' length of stay and turnover rate were determined. 
The results of RM-ANOVAs indicated that new graduates' clinical competency significantly increased over time, as did the aspects of their role adjustment concerning familiarity with work demands and the hospital environment. In addition, the nurses' demonstrated a significant increase in their level of involvement job satisfaction which deals with having the authority to share in the determination of methods and procedures, setting goals, and directing others on the job. However, subjects' scores significantly declined on the NCS portion of role adjustment which pertains to their perceptions of the actual practice of professional nursing. No significant changes over time were detected for the new graduates' overall job satisfaction, nor for their intrinsic, interpersonal, or extrinsic satisfaction. In addition, there were no significant differences between stayers and leavers on the outcome variables of clinical competency, role adjustment, or job satisfaction.

Subjects' length of stay was slightly higher than the average length of stay of new graduates at the hospital study site prior to implementation of the transition program. In addition, compared to the pre-program neophyte turnover rate, nurses in the study had a substantially lower rate of attrition.

This study also investigated the professional and role-oriented values possessed by the new graduate nurses. Factor analyses performed on the NCS and the RFS instruments resulted in a three-factor solution and a five-factor solution, respectively. The factor structures revealed that subjects' strongest values were associated with dimensions of personal and professional benevolence, growth, and opportunity, and working effectively with others and within the hospital system. RM-ANOVAs conducted 
on the factor scores retained from the factor analyses indicated that new graduates' values significantly decreased over time with respect to intrinsic and extrinsic rewards and stress and autonomy. However, subjects' values related to organizational relationships and roles, as well as professional development and clinical practice, increased significantly over time. There were no significant differences between stayers and leavers with respect to their professional and role-oriented values. 


\section{Chapter V}

\section{DISCUSSION}

\section{Overview}

Major changes in the structure of health care delivery and increasing pressure to reduce costs are challenging hospitals to increase their attention to the causes and consequences of staff nurse turnover. Premature turnover and low retention rates among staff nurses in hospitals have been found to be seriously detrimental to quality patient care, industry competitiveness, personnel productivity, and professional enhancement. The human and financial consequences to the patient, the nurse, and the hospital that are associated with staff nurse turnover necessitate that all nursing resources be protected. This is especially true as the demand for nurses outpaces the supply. Nursing researchers and administrators must continue to be concerned with factors that contribute to the employment stability of both experienced and new graduate practitioners. However, studies regarding the turnover of neophyte nurses in particular are especially warranted since new graduates are the original source for the needed supply of experienced practitioners. Furthermore, interventions which support the new graduate's transition into and continued employment with hospitals must be investigated and their outcomes evaluated in order to justify their selection and implementation.

The initial work of Kramer, Schmalenberg, and Benner regarding new graduates' entry into the hospital work force has been shown to be highly relevant in today's environment. Specifically, this original body of literature brought into focus a problem which is receiving increasing attention in the current organizational literature. That is, 
genuine attention to employees' values, and critical examination of the extent to which organizational culture and organizational structures are congruent with and supportive of employees' values. Moreover, the current organizational literature indicates that the experience of "reality shock" is not a phenomenon unique to nurses, and that attention to the needs of professionals during transition to and tenure with an organization is at the least a critical factor in organizational success, if not a moral imperative.

The nursing literature suggests that the implementation of neophyte transition programs will prevent or ameliorate the values and role conflicts new graduate nurses experience upon entry into hospital employment, and in turn reduce premature turnover and increase retention. If this is true, then the effort and costs expended in the design and implementation of such programs would offset the serious and extensive negative outcomes associated with denial of the problems new graduates' face in their transition from education to service or with selection of ineffective responses to these problems. However, nursing research on neophyte transition programs has yielded mixed and inconclusive results, and it is often weakened by methodological inadequacies.

This study utilized secondary data, obtained using a longitudinal, panel study design, to examine whether 890 new graduate nurses' clinical competency, role adjustment, job satisfaction, and length of stay increase, and their rate of turnover decreases, as a result of their participation in a transition program. In addition, the data were analyzed to determine if, among new graduates participating in the transition program, there are differences with regard to clinical competency, role adjustment, and job satisfaction between those who stayed beyond 15 months after hire (stayers) and those 
who terminated employment within 15 months (leavers). Relatedly, this study sought to identify the professional and role-oriented values the new nurses possessed upon initial employment, to determine whether these values changed over time, and to discern whether differing values affected the nurses' length of stay. Repeated measures analyses of variance (RM-ANOVA) were utilized to examine changes over time in subjects scores on the outcome variables, and factor analysis was used to identify the new graduates' values. A summary and discussion of the results, as well as the limitations and implications of the study, and recommendations for future research are presented in this chapter.

\section{Interpretations}

The results of RM-ANOVAs indicated that new graduates' clinical competency significantly increased over the three time points. That is, subjects' clinical competency rose from a moderate level of proficiency at the time of initial employment to moderately high level of proficiency 12 months later. These findings lend support to the results of previous studies on transition programs' positive effect on neophytes' clinical competency (Kasprisin \& Young, 1985; Kramer \& Schmalenberg, 1978; Mims, 1984; Ressler et al., 1991; Weiss, 1988; Weiss \& Ramsey, 1977). However, issues of carry-over effects associated with data collection using the same instruments across multiple time points may also be a factor in these results. Furthermore, the extent to which the transition program per se, versus the passage of time concomitant with the opportunity to experience the repetition of clinical tasks, brought about the nurses' increase in clinical competencies cannot be determined. 
With respect to role adjustment, subjects' moderately high degree of confidence regarding familiarity with work demands and the hospital environment, as reported on the RFS, increased significantly over time. In contrast, however, on the NCS, which pertains to subjects' perceptions of the actual practice of professional nursing, the nurses' scores demonstrated a significant decline. These contrasting results may be explained in several ways. First, familiarity with work and the environment may be a direct function of time spent on the job. Second, the program's seminars may have emphasized organizational and role functioning more than professional role clarification and strengthening. Third, demographic information revealed that $80.4 \%$ of the sample not only worked to some extent during nursing school but also that $61 \%$ of the employment was in nursing positions, with more than $17 \%$ of the subjects working as nurse externs at the study hospital. Thus, the improved scores on the RFS may also be explained by prior exposure to hospital work settings in general, and prior employment in the study site hospital in particular. Last, it is possible that these contrasting results reflect phenomena which are similar to other studies' findings that neophytes' bureaucratic role scores increase while their professional role scores decrease (Kramer, 1968; Weiss, 1984). In other words, although the NCS scores reflected moderately positive perceptions at both $t_{1}$ and $t_{3}$, the significant decline in scores observed across the groups indicates that the nurses felt increasingly negative about professional nursing in general, and their professional role in particular, while the increase in RFS scores indicates feelings of increasing confidence in navigating job requirements and the hospital system. These findings would therefore suggest that the transition program was not effective in 
sustaining or developing the new graduates' sense of professionalism nor their professional role, and thus refute the conclusions drawn by Kramer \& Schmalenberg (1978) and Weiss \& Ramsey (1977) regarding the benefits of transition programs on professional role conception. However, as Aldrich (1988) and Dear et al. (1982) have suggested, the effects of a transition program on neophyte nurses' role adjustment in general, and their professional role conception in particular, may not emerge for several years. If this is the case, then long-term followup and measurement of individuals' service and professional role conceptions would be warranted.

On the outcome measure of job satisfaction, no significant changes over time were detected for overall job satisfaction, nor for the subscales concerning intrinsic, interpersonal, or extrinsic satisfaction. Although the nurses' total satisfaction scores were moderately high upon employment as well as one year later, a downward trend in these scores was evident. On the involvement subscale, however, subjects' demonstrated a significant increase, indicating improved satisfaction with their authority to share in the determination of methods and procedures, setting goals, and directing others on the job. These findings are consistent with the results of previous studies reported in the literature, especially those in which professional and bureaucratic role conceptions are also measured (Weiss, 1984; Weiss \& Ramsey, 1977). In those studies, the investigators reported that increases in this dimension of satisfaction coincided with increases in a service, or bureaucratic, role conception. This is also true in this study given the findings related to the RFS data as discussed above. In addition, Gibbons \& Lewison (1980) found that decreases in the intrinsic and interpersonal aspects of job satisfaction 
accompanied decreases in the professional role orientation. In this study, subjects' increasingly negative perceptions of professional nursing practice occurred with decreases also in satisfaction with utilizing skills and abilities, doing important and worthwhile things, realizing their potential, and rewarding relationships with others. The literature on transition programs and subjects' nonsignificant scores in this study, suggest that although such programs may not bring about improved job satisfaction on the majority of dimensions measured, they may prevent significant declines in new nurses' overall satisfaction with their work. Another interpretation of these findings may be that significant increases in the subjects' involvement scores concomitant with nonsignificant decreases in the total, intrinsic, and interpersonal scores are indicative of the nurses' efforts to integrate their professional and service roles. According to reality shock theory, the bicultural nurse acquires the ability to blend both her professional and bureaucratic roles. Thus, the results of this study may reveal that subjects' were integrating their service and professional roles successfully enough that their increasing and decreasing scores, respectively, balanced one another and resulted in a stable and rather positive overall job satisfaction score.

The study's findings regarding the new graduates' longer length of stay (LOS) and lower tumover rate (TOR), as compared to pre-program LOS and TOR, suggest that the transition program was effective in increasing the nurses' retention. In addition, compared to national data on neophyte nurses' average LOS for the early 1980's which was 2.3 years (Health Care Advisory Board, 1987), the nurses in this study, for the years 1983 through 1985, had an average LOS of 3.5 years. With regard to TOR, this study's 
findings are also consistent with those of Holloran et al. (1980), Hollefreund et al. (1981), and Rufo (1984) where post-program implementation TOR was lower than preprogram TOR. However, the TOR findings in this study are less clear when compared to state and national data for this same period of time. For the state where the study site hospital is located, the 1980,1982 , and 1985 TORs were $25 \%, 23.6 \%$, and $18.5 \%$, respectively (Final Report, 1987). The TOR for nurses nationally was $30 \%$ in 1980 , $23 \%$ in 1982, and $18 \%$ in 1984 (Health Care Advisory Board, 1987). In this study, nurses' TOR was $21.9 \%$ in $1983,29.9 \%$ in 1984 , and $36.4 \%$ in 1985 (see Table 10). Thus, although the TOR for the 1983 panel year was lower than those reported at both the state and national level, the 1984 and 1985 panel years' TOR was substantially higher. In should be noted, however, that the state and national data reflect the TOR of registered nurses in general and not new graduate nurses in particular. If the national neophyte TOR of 50\% cited by some authors (Final Report, 1987) is used as the point of comparison, then clearly the TORs for the 1983-1985 panel years are significantly less. Although it is clear that LOS and TOR improved at the study site hospital after the implementation of the transition program, the extent to which local and national macroeconomic factors influenced this improvement is unknown. Given the severe recession experienced throughout the nation in the early 1980's, the improved LOS and TOR found in this study may reflect the nurses' need to enter and remain in the work force in order to contribute essential income for their families.

An interesting finding in this study involves the vast difference between stayers and leavers with regard to their respective LOS, concomitant with the significant 
difference between the groups on the demographic variable regarding intended length of stay (see Table 5). Nurses in the stayers cohort had a considerably longer average LOS $(M=47.58$ months, $m d n=40.00, S D=28.87)$ than nurses in the leavers group $(M=3.85$ months, $m d n=5.71, S D=4.97$ ). Since this study found that there were no significant differences between stayers and leavers with respect to the outcome variables of clinical competency, role adjustment, and job satisfaction, the groups' difference in LOS may be related to an "intent to leave" or "intent to stay" disposition held by subjects prior to employment. Such variables have been suggested by other researchers investigating the causes of nurse turnover. In fact, several studies have found that intent to leave, or anticipated turnover, is not only a significant intervening variable but also a major predictor of actual turnover among nurses (Curry, Wakefield, Price, Mueller, \& McCloskey, 1985; Hinshaw, Smeltzer, \& Atwood, 1987; Parasuraman, 1989; Weisman, Alexander, \& Chase, 1981). In addition, these investigators have hypothesized that more obvious differences between stayers and leavers may emerge later in the process of employment, and that at such time group differences with regard to performance, role conception, and job satisfaction may appear.

This study also sought to identify the professional and role-oriented values new nurses possessed upon initial employment. Factor analyses performed on the NCS and the RFS instruments revealed that subjects' strongest values were associated with dimensions of personal and professional benevolence, growth, and opportunity, and working effectively with others and within the hospital system. These two values accounted for the largest amount of the variance within their respective factor structures, 
thus illustrating the predominance among the subjects of: 1) an intrinsically driven professional and personal value system; and 2) a strong value orientation towards positive and cooperative working relationships with others as well as constructive interaction with the organization. These findings support prior research on employee values which has indicated that professionals in general, and new graduates in particular, are concerned with their ability to function within an organization in a manner that supports both their professional and service values. In addition, these results are consistent with the findings of an ANA survey of RNs working in hospitals which found that the four workplace issues of greatest importance were quality of patient care, adequate staffing, professional regard, and professional autonomy (Alspach, 1991). However, this study's findings may refute to some extent Raelin's (1991) contention that professionals are more strongly guided by loyalty to their professional values rather than by their service or organizational values. The factor analysis performed in this study demonstrated that the factor representing organizational relationships accounted for only a slightly higher percentage of variance in the RFS factor solution than the factor representing personal and professional intrinsic reward explained in the NCS factor structure, $37 \%$ and $35 \%$ respectively.

RM-ANOVAs conducted in order to determine whether these values changed over time indicated that new graduates' values related to organizational relationships and roles, as well as professional development and clinical practice, increased significantly. However, significant decreases over time were found for subjects' values pertaining to intrinsic and extrinsic rewards and stress and autonomy. These changes suggest that 
during the first year of employment new graduates' service, or bureaucratic, role values increased, but their professional role values decreased. In other words, as new nurses became increasingly concerned with their organizational and clinical practice functioning and development, their perception that nursing would offer personal and professional rewards, career opportunities and income, and professional autonomy declined. These findings are consistent with those of previous studies' on new graduates' change in role conception and values, in particular the investigations conducted by Kramer (1968, 1969a), Speeding et al. (1981), Ahmadi et al. (1987), and Green (1988). However, given subjects' relatively positive and stable satisfaction scores, improved LOS and TOR, the absence of significant differences between stayers and leavers with respect to their professional and role-oriented values, and the increasing service values concomitant with the decreasing professional values, these findings may provide additional evidence of this sample's bicultural character. As noted previously in this discussion, the nurses in this study may be demonstrating the ability to integrate the professional and bureaucratic values required when employed as professionals in traditional service organizations.

Another explanation for these findings may be related to elements associated with the hospital's culture and organizational structure. Many factors that are believed to be instrumental in influencing employees values, roles, satisfaction, and length of stay were not measured or controlled for in this study. Therefore, it is possible, if not probable, that the constellation of results found in this study are directly influenced by such unknown organizational factors. For instance, perhaps there was a good match between the hospital's culture and its structure and this cohort of nurses. In particular, the new 
graduates may have experienced an alignment in their values with certain organizational policies, procedures and rewards, or with other disciplines' personnel, or with other departments' operations. Moreover, the implementation of the transition program itself, as well as the specific protocols of the program, may have been perceived by the new graduates as positive and valuing gestures on the part of hospital and nursing administration towards the nurses. Such gestures can symbolize to the neophyte that administration values and cares about their successful and positive transition to employment as well as their overall work experience. Furthermore, elements of the transition program's design, such as the frequent contact with head nurses and clinical instructors, the regular contracting for clinical skill development, and the seminars not only attend to the needs of the new graduate but also convey that the nurses' individual needs are driving the process rather than just the needs of the institution.

\section{Strengths and Limitations}

The strengths of this study are found in the data's statistical power due to the large aggregate sample size, the relative stability of the transition program's protocols, and the longitudinal nature of its design which provided for not only multiple measures on individual subjects over time but also the inclusion of successive panel groups over nine years.

However, this study also exhibits several limitations. First, the study results are not generalizable beyond the accessible population at the medical center where the data were collected due to the self-selecting nature of the convenience sample. Subjects 
included in the study selected employment at the study site, and in turn their participation in the transition program.

Second, there were substantial missing data, especially involving data collection at the third time point. In particular, the 1988 group lacked data on all the outcome measures at the third time point, and there were only one to two cases in the 1991 group with complete JSI data at $t_{3}$. Examination of the data and knowledge of the data collection methods reveal that this limitation resulted from a combination of subject attrition, declining retum rate of the questionnaires over time, and lapses in provisions for distribution and collection of questionnaires. These factors caused not only uneven cell counts but also inadequate sample sizes with respect to the panel year groups, and consequently interfered with the planned statistical procedures (i.e., RM-ANOVAs).

Third, although the changes in the transition program between 1987 and 1988 were relatively minor, and between-group RM-ANOVAs as well as one-sample RMANOVAs do not indicate significant differences after the 1987-1988 modifications, the longitudinal nature of the study and thus its findings may have been compromised by this disruption in protocols.

Fourth, the data collection instruments used in this study, in particular the NCA and the RFS, have not benefitted from repeated use in research and therefore their psychometric properties are not extensively established. Although these instruments had acceptable validity and reliability coefficients when used previously (Final Report, 1987), additional evidence of their psychometric qualities are not documented. 
Fifth, concern may be raised as to the appropriateness of using data collected with the NCS and RFS for the identification of the subjects' underlying professional and roleoriented values. Although these instruments were designed to measure the attributes of professional nursing practice and role conception, respectively, the RFS psychometric qualities are not well established and it cannot be assured at this time that either instrument has the ability to survey nurses' values in a valid or reliable manner. Therefore, this study's findings regarding the new graduates' values should be accepted with caution.

Last, the findings of this study, especially those related to the nurses' values, may be constrained by the use of only quantitative measurements on the dependent variables. Supplemental qualitative data concerning subjects' changes over time on the outcome variables would have provided additional scope and depth about the nurses' experience in the transition program and their value orientations. In addition, information on various organizational factors and the hospital's structure which reflect the system's culture would have provided important dimensions to the interpretation of the study test results as well as have illuminated the interrelationships among the study's variables. Given the absence of this kind of qualitative data, it is unknown in what manner, or to what degree, the transition program and/or the hospital environment met the professional and personal core needs of the new graduates or supported their individual visions.

\section{Implications}

The results of this study suggest that the transition program was effective in promoting the new graduates' increase in clinical proficiency and service role 
functioning, and in sustaining a positive degree of job satisfaction. In addition, the study's findings suggest that the transition program was effective in increasing the length of stay and lowering the tumover rates for new graduates at the study hospital. However, the study does not provide evidence that the transition program was effective in promoting the new graduates' professional role development. Rather, the results indicate that either the nurses' declining sense of professionalism was moderated by an increasing service orientation; or, that the consequences of their increasingly negative perceptions of professional nursing in general, and their professional role in particular, were not apparent within the time frame of data collection. Nevertheless, the results of this study have important implications for nursing education, practice, management, research and knowledge development.

The findings of this study can inform nursing educators as to the strategies, activities, and behaviors that facilitate new practitioners in achieving higher levels of clinical competency and that support their role adjustment from student to professional. Curriculum design and classroom and clinical activities must not only allow for coverage of theoretical material but also provide for sufficient time and opportunities for students to practice simulated and real patient care skills. Resources must be found and allocated to provide students with clinical skills labs that are equipped with appropriate and current technology. Students also need clinical experiences where they have the opportunity to perform patient care activities on multiple occasions and to take care of larger numbers of patients. In addition, nursing educators need sufficient resources to provide alternative learning methods such as videotapes and self-paced and computer-assisted modules. 
Furthermore, although "reality shock" is not the buzz word today that it was some years ago, no one can deny that the phenomena remains a current problem for most new practitioners. Therefore, nursing educators must incorporate into their curricula content which addresses the causes and consequences of transition problems as well as realistic suggestions of methods that new graduates can use to cope with their new roles. One obvious strategy would be through the development of mentoring relationships with students in which faculty share their own transition experiences and assist the student in developing realistic conceptions of herself both as student nurse and future professional nurse. Another strategy must be that schools of nursing create cultures where students as novice practitioners are valued, respected, and treated with regard. In addition, faculty must have credibility as clinical instructors and nursing professionals. We cannot expect new nurses to enter initial employment with a sense of professionalism, respect, and self-esteem for themselves or others, including patients, if they have not received such while as students. The findings of this study with respect to the outcome variable of role adjustment and the nurses' change in values suggest that efforts such as these are especially needed.

From a nursing practice perspective, nursing students and new graduates, perhaps with the assistance of faculty, should use this body of research in selecting hospitals for their initial employment. Potential workplaces should be evaluated as to their orientation models, existence of transition programs, support programs, educational resources, and continuing education opportunities. In addition, information should be acquired during conversation and interviews as to the nature and frequency of clinical supervision and 
evaluation, and whether there are provisions for unit-level preceptors. Similarly, nurses in clinical instructors' and preceptors' positions in hospitals who have responsibility for or contact with new graduates should become familiar with this body of research so that, like educators in schools of nursing, they too can implement the strategies, activities, and behaviors found to be supportive and facilitative of new practitioners. In doing so, particular attention should be paid to the results concerning the new graduates' role adjustment. The protocols investigated in this study were extensive both in scope and depth in the areas of clinical skills and competency achievement. However, it appears that the seminars designed to address role adjustment were not sufficient in frequency, content, or methods to produce the desired effects. Consequently, those in hospital positions responsible for transition programs or professional development seminars should consider new or additional ways to address this aspect of the new nurses' transition.

The implications of this study for nursing management are multi-fold. First, nursing administrators and unit-level managers must not be so consumed with the retention of the experienced nurse that they overlook the importance of retaining the new graduate. In that light, nursing administrators and managers must continue to assess their institutions, departments, and units for organizational processes that are not supportive or facilitative of the new graduate's growth and development both clinically and professionally. This includes the implementation of transition type programs, preceptorships, and professional continuing education opportunities.

Second, they must implement organizational processes that are congruent with the values of professional nursing practice. This requires structures, operations, and 
relationships that allow and facilitate nurses to practice to their fullest capacity with respect, regard, and appropriate tangible rewards. Although the magnet hospitals study and similar research on hospitals utilizing decentralization, career ladders, and governance structures have provided nursing administrators with specific strategies found to be successful in recruiting and retaining nurses, serious consideration should also be given to the organizational literature reviewed in this study. In particular, the literature pertaining to the continued dominance of the bureaucratic paradigm (Clark, 1985) and the non-orthodox alternatives to organizing suggested by Clark \& Meloy (1988), Kofman \& Senge (1993), and Weick $(1979,1985)$ should be helpful to nurses as they try to think in new ways about their current organizations and envision changes for the future.

Third, nurses in hospitals need to systematicaliy and objectively evaluate the recruitment and retention programs that are currently in place. Whether such programs are targeted to new graduates or experienced nurses or both, the actual outcomes associated with them must be identified. With well designed program evaluation plans and the resultant data, nursing administrators are better able to make judicious decisions about program modifications and resource allocation. Moreover, with such documentation, nursing leaders are in a stronger position to receive the needed support from hospital administrators, physicians, and other disciplines to make changes in organizational processes that will create successful and value-congruent workplaces for nurses.

The implications of this study for nursing research and knowledge development are also apparent. First, given the continued research in the area of new graduates' 
transition from school to employment it is it clear that the theoretical framework advanced by Kramer and her colleagues remains an area of interest to both researchers and hospital nurse administrators. Thus, this study has provided new and additional testing of the framework and thereby contributes to the body of knowledge articulated to date regarding neophyte nurses' transition from academe to employment. Second, this study has demonstrated that Kramer and others were ahead of their times when they initially expressed their concerns about nurses experiencing professional-bureaucratic role conflicts. But more importantly, the current nursing and non-nursing organization literature on employee values, organizational culture, and organizational structure clearly indicates that continued research in this area is not only highly relevant but also critically needed. Today's social, economic, technologic, and health care delivery requirements demand that attention be paid to protecting all nursing resources. Therefore, investigations which focus on methods for promoting and retaining the supply of registered nurses are strongly indicated; and their importance underscores the significance of this study's examination of new graduates' values, their change over time, and their relationship to the nurses employment tenure. It is only by preserving the retention of the new graduate nurse that we will be able to maintain an adequate supply of experienced nurses.

\section{$\underline{\text { Recommendations }}$}

The results and limitations of this study give direction as to recommendations for future research. First, given the change in this study's protocols after 1986, the secondary data used could be further analyzed by examining whether there are 
differences between the 1983-1986 panel groups and the 1989-1991 groups. These analyses would provide additional information as to the role that the change in program protocols may have on the outcome measures. Second, statistical analyses such as multiple regression, cluster analysis, and discriminate analysis, also using this data set may assist in identifying the factors that most significantly contributed to the nurses longer length of stay, and in discerning more subtle similarities among and differences between stayers and leavers. Third, and related to the preceding, the findings of the present study would be further illuminated if additional tests were performed which examined the relationships between the outcome variables and specific demographic measures, such as the type and amount of employment during school, the length of time subjects' intended to work in a hospital setting, and subjects' basic nursing education.

Other than additional research using this study's data, this investigation has also demonstrated the need for further research on transition programs in general. Ideally, such research should also be longitudinal in design and include mechanisms for comparison with control groups, for instance, matched designs using new graduates who do not participate in a transition program. Preferably, the matched controls would be employed in the same institution so that the influence of organizational factors can be accounted or controlled for. Similarly, further research on transition programs and nurses' values should include qualitative methods in order to elucidate important personal and organizational factors that do not lend themselves to quantitative measurement. As evident in this study and previously discussed, further investigation of nurses' values is 
strongly recommended, yet research in this area would require sound and well established quantitative approaches with supplemental qualitative data.

The last recommendation is for research which focuses on the outcomes associated with organizational structures currently advocated in the nursing and non-nursing organizational literature purporting to enhance the clinical functioning, role adaptation, and job satisfaction of nurses in hospital settings. Such programmatic structures may help us identify ways of designing work places that approach the Y-Y concept of organizing advocated by Clark \& Meloy (1988). And as mentioned previously in this discussion, research of this nature must consider social, geographic, family, and economic factors that may influence nurses' performance, perceptions, and tenure.

\section{Summary}

The findings of this study provide further evidence that a neophyte transition program can be a sound and judicious strategy for hospitals to implement as an aid to new graduates' in their transition from school to professional practice. The results suggest that the transition program was effective in supporting the new graduates' increase in clinical proficiency and role adjustment, and in sustaining a positive degree of job satisfaction. In addition, the study's findings suggest that the transition program promoted longer length of stay and lower tumover rates for the new graduates at the study hospital. Recommendations are made, however, for additional research related to organizational factors, collected through naturalistic methods of inquiry, as well as information on social and economic factors, which would provide critical perspectives to interpretation of the study's findings and assist in the formulation of more conclusive 
results with regard to transition programs and nurses' professional and role-oriented values. 


\section{REFERENCES}

Ahmadi, K. S., Speedling, E. J., \& Kuhn-Weissman, G. (1987). The newly hired hospital staff nurse's professionalism, satisfaction, and alienation. Intermational Joumal of Nursing Studies, 24(2), 107-121.

Aldrich, S. K. (1988). A neurosciences internship and graduate nurse role conception. Joumal of Neuroscience Nursing, 20(6), 377-385.

Alexander, J. W. (1989). Programs of administrative research for nursing administration. In B. Henry, C. Amdt, M. Di Vincenti \& A. Marriner-Tomey (Eds.), Dimensions of nursing administration; Theory, research, education, and practice (p. 267-284). Boston: Blackwell Scientific Publications.

Alspach, J. G. (1991). Workplace issues for nurses: A holiday wish list. Critical Care Nurse, $\underline{11}(10), 8,10$.

Bame, S. I. (1993). Organizational characteristics and administrative strategies associated with staff tumover. Health Care Management Review, 18(4), 70-86.

Benner, P. (1974). Reality testing a reality shock program. In M. Kramer, Reality Shock: Why nurses leave nursing (p. 191-215). Saint Louis: The C. V. Mosby Company.

Benner, P. \& Benner, R. V. (1979). The new nurse's work entry: A troubled sponsorship. New York: The Tiresias Press, Inc.

Blegen, M. A. \& Mueller, C. W. (1987). Nurses' job satisfaction: A longitudinal analysis. Research in Nursing \& Health, 10, 227-237.

Bray, J. H. \& Maxwell, S. E. (1985). Multivariate analysis of variance. Newbury Park: Sage Publications.

Burns, N. \& Grove, S. K. (1987). The practice of nursing research: Conduct, critique and utilization. Philadelphia: W. B. Saunders Co.

Caroselli, C. (1992). Assessment of organizational culture: A tool for professional success. Qrthopaedic Nursing, 11(3), 57-63.

Cavanagh, S. J. (1989). Nursing tumover: literature review and methodological critique. Joumal of Advanced Nursing, 14, 587-596. 
Clark, D. L. (1985). Emerging paradigms in organizational theory and research. In Y. S. Lincoln (Ed.), Organizational theory and inquiry: The paradigm revolution (p. 43-78). Newbury Park, CA: Sage Publications, Inc.

Clark, D. L. \& Meloy, J. M. (1988). Renouncing bureaucracy: A democratic structure for leadership in schools. In T. Sergiovanni \& J. Moore (Eds.), Schooling for tomorrow: Directing reforms to issues that count (p. 272-294). Boston: Allyn \& Bacon.

Coeling, H. V. E. (1990). Organizational culture: Helping new graduates adjust. Nurse Educator, 15(2), 26-30.

Coeling, H. V. E. \& Simms, L. M. (1993a). Facilitating innovation at the nursing unit level through cultural assessment: How to keep management ideas from falling on deaf ears, Part 1. Joumal of Nursing Administration, 23(4), 46-53.

Coeling, H. V. E. \& Simms, L. M. (1993b). Facilitating innovation at the nursing unit level through cultural assessment: How to keep management ideas from falling on deaf ears, Part 2. Joumal of Nursing Administration, 23(5), 13-20.

Coeling, H. V. E. \& Wilcox, J. R. (1988). Understanding organizational culture: A key to management decision-making. Joumal of Nursing Administration, 18(11), 16-24.

Cowart, M. E. \& Serow, W. J. (Eds.). 1992. Nurses in the workplace. Newbury Park: Sage Publications.

Craver, D. M. \& Sullivan, P. P. (1985). Investigation of an internship program. Journal of Continuing Education in Nursing, 16(4), 114-118.

Curran, C. R. \& Miller, N. (1990). The impact of corportate culture on nurse retention. Nursing Clinics of North America, 25(3), 537-549.

Curry, J. P., Wakefield, D. S., Price, J. L., Mueller, C. W., \& McCloskey, J. C. (1985). Determinants of turnover among nursing department employees. Research in Nursing \& Health, $\underline{8}, 397-411$.

Deal, T. E. \& Kennedy, A. A. (1982). Corporate cultures: The rites and rituals of corporate life. Reading, MA: Addison-Wesley Publishing Co.

Dear, M. R., Celentano, D. D., Weisman, C. S., \& Keen, M. F. 91982). Evaluating a hospital nursing internship. Journal of Nursing Administration, 12(11), 16-20. 
del Bueno, D. J. \& Vincent, P. M. (1986). Organizational culture: How important is it? Journal of Nursing Administration, 16(10), 15-20.

DePree, M. (1989). Leadership is an art. New York: Doubleday.

Final Report. (1987). Unpublished document. Prepared by staff at the original study site hospital.

Fleming, B. W., Woodcock, A. G., \& Boyd, B. T. (1975). From student to staff nurse: A nurse internship program. American Journal of Nursing $75(4), 595-$ 599.

Gardner, D. L. (1992). Conflict and retention of new graduate nurses. Western Journal of Nursing Research, 14(1), 76-85.

Gardner, J. W. (1990). On leadership. New York: The Free Press.

Garner, J. F., Smith, H. L., \& Piland, N. F. (1990). Strategic nursing management: Power and responsibility in a new era. Rockville, Maryland: Aspen Publications.

Gibbons, L. K. \& Lewison, D. (1980). Nursing internships: A tri-state survey and model for evaluation. Journal of Nursing Administration, 10(2), 31-36.

Green, G. J. (1988). Relationships between role models and role perceptions of new graduate nurses. Nursing Research, 37(4), 245-248.

Grey, R. J. \& Gelfond, P. A. (1990). The people side of productivity: Responding to changing employee values. National Productivity Review, 9(3), 301-312.

Hartshorn, J. C. (1992). Evaluation of a critical care nursing internship program. Journal of Continuing Education in Nursing, $\underline{23}(1), 42-48$.

Health Care Advisory Board. (1987). Nurse recruitment and retention: Current hospital strategies. Washington, DC: The Advisory Board Company.

Hinshaw, A. S., Smeltzer, C. H., \& Atwood, J. R. (1987). Innovative retention strategies for nursing staff. Journal of Nursing Administration, 17(6), 8-16.

Hollefreund, B., Mooney, V. M., Moore, S., \& Jersan, J. (1981). Implementing a reality shock program. Journal of Nursing Administration, 11(1), 16-20.

Holloran, S. D., Mishkin, B. H., \& Hanson, B. L. (1980). Bicultural training for new graduates. Nurse Educator, $\underline{5}(1), 8-14$. 
Hunter, E. P., Pollman, J., \& Moore, B. (1990). An internship program's influence on recruitment and retention. Journal of Nursing Staff Development, $\underline{6}(6), 302-$ 304.

Johnston, C. L. (1991). Sources of work satisfaction/dissatisfaction for hospital registered nurses. Western Journal of Nursing Research, 13(4), 503-513.

Jones, C. B. (1990). Staff nurse turnover: Part II, measurements and results. Journal of Nursing Administration, 20(5), 27-32.

Jones, C. B. (1992). Calculating and updating nursing turnover costs. Nursing Economics, 10(1), 39-45.

Kanter, R. M. (1983). The change masters: Innovation and entrepreneurship in the american corporation. New York: Simon \& Schuster.

Kanter, R. M. (1989). When giants leam to dance: Mastering the challenge of strategy, management, and careers in the 1990s. New YorK: Simon and Schuster.

Kasprisin, C. A. \& Young, W. B. (1985). Nurse internship program reduces turnover, raises commitment. Nursing \& Health Care $6(3), 137-140$.

Kennerly, T. Professional values in health care. Health Services Management, 88(3), 19-21.

Kleinbaum, D. G., Kupper, L. L., \& Muller, K. E. (1988). Applied regression analysis and other multivariate methods (2nd ed.). Boston: PWS-Kent Publishing Company.

Kofman, F. \& Senge, P. M. (1993). Communities of commitment: The heart of learning organizations. Organizational Dynamics, 22(2), 5-23.

Kohler, H. (1988). Statistics for business and economics (2nd ed.). Glenview, IL: Scott, Foresman and Company.

Kotecki, C. N. (1992). Nursing internships: Taking a second look. Journal of Continuing Education in Nursing, 23(5), 201-205.

Kramer, M. (1968). Role models, role conceptions, and role deprivation. Nursing Research, 17(2), 115-120.

Kramer, M. (1969a). New graduate speaks--again. American Journal of Nursing, 69(9), 1903-1907. 
Kramer, M. (1969b). Collegiate graduate nurses in medical center hospitals: Mutual challenge or duel. Nursing Research, 18(3), 196-210.

Kramer, M. (1970). Role conceptions of baccalaureate nurses and success in hospital nursing. Nursing Research, 19(5), 428-439.

Kramer, M. (1974). Reality shock: Why nurses leave nursing. Saint Louis: The C. V. Mosby Company.

Kramer, M. (1985). Why does reality shock continue? In J. C. McCloskey \& H. K. Grace (Eds.), Current Issues in Nursing (2nd ed., p. 891-903). Boston: Blackwell Scientific Publications.

Kramer, M. (1990a). Trends to watch at the magnet hospitals. Nursing90, 20(6), 6774.

Kramer, M. (1990b). The magnet hospitals: Excellence revisited. Journal of Nursing Administration, 20(9), 35-44.

Kramer, M. \& Baker, C. (1971). The exodus: Can we prevent it? Journal of Nursing Administration, 1(3), 15-30.

Kramer, M. \& Hafner, L. P. (1989). Shared values: Impact on staff nurse job satisfaction and perceived productivity. Nursing Research, 38(3), 172-177.

Kramer, M. \& Schmalenberg, C. (1977). The first job-a proving ground: Basis for empathy development. Journal of Nursing Administration, 7(1), 13-20.

Kramer, M. \& Schmalenberg, C. (1978). Bicultural training and the new graduate role transformation. Nursing Digest, $5(4), 1-47$.

Kramer, M. \& Schmalenberg, C. (1988a). Magnet hospitals: Institutions of excellence, Part 1. Journal of Nursing Administration, 18(1), 13-24.

Kramer, M. \& Schmalenberg, C. (1988b). Magnet hospitals: Institutions of excellence, Part 2. Journal of Nursing Administration, 18(2), 11-19.

Kramer, M. \& Schmalenberg, C. (1991a). Job satisfaction and retention: Insights for the 90's, Part 1. Nursing91, 21(3), 50-55.

Kramer, M. \& Schmalenberg, C. (1991b). Job satisfaction and retention: Insights for the 90's, Part 2. Nursing91, 21(4), 51-55. 
Lewison, D. \& Gibbons, L. K. (1980). Nursing internships: A comprehensive review of the literature. Journal of Continuing Education in Nursing, 11(2), 32-38.

Liodtka, J. M. (1992). Formulating hospital strategy: Moving beyond a market mentality. Health Care Management Review, 17(1), 21-26.

Marchette, L. \& Merker, A. (1985). The effect of a nurse internship program on novice nurses' self-evaluation of clinical performance. Journal of Nursing Administration, 15(5), 6-7.

Martel, G. D. \& Edmunds, M. W. (1972). Nurse-internship program in Chicago. American Journal of Nursing, 72(5), 940-943.

May, L., Minehan, P. L., \& Deluty, L. (1981). Evaluating bicultural training. Journal of Nursing Administration, 11(5), 24-29.

McCloskey, J. (1974). Influence of rewards and incentives on staff nurse turnover rate. Nursing Research, 23(3), 239-247.

McCloskey, J. C. \& Grace, H. K. (1990). Current Issues in Nursing (3rd ed.). Boston: Blackwell Scientific Publications.

McClure, M., Poulin, M., Sovie, M., \& Wandelt, M. (1982). Magnet hospitals: Attraction and retention of professional nurses. Kansas City: American Nurses Association.

McDaniel, C. \& Stumpf, L. (1993). The organizational culture: Implications for nursing service. Lournal of Nursing Administration, 23(4), 54-60.

Mims, B. C. (1984). A critical care internship program. Dimensions of Critical Care Nursing, $3(1), 53-59$.

Minnick, A., Roberts, M.J., Curran, C.R., \& Ginzberg, E. (1989). What do nurses want? Priorities for action. Nursing Outlook, 37(5), 214-218.

Morgan, G. (1986). Images of organization. Newbury Park, CA: Sage Publications, Inc.

Munson, F. \& Heda, S. (1974). An instrument for measuring nursing satisfaction. Nursing Research, 23(2), 159-166.

National League for Nursing. (1979). Employment, mobility, and personal characteristics of nurses newly licensed in 1977. [Publication No. 19-2000 (DS7901)]. New York: National League for Nursing. 
Norusis, M. J. (1988). SPSS/PC+ advanced statistics, v2.0. Chicago: SPSS, Inc.

Nyberg, J. (1991). The nurse as professnocrat. Nursing Economics, 9(4), 244-247, 280.

Parasuraman, S. (1989). Nursing turnover: An integrated model. Research in Nursing \& Health, 12, 267-277.

Peters, T. J., \& Waterman, R. H. (1982). In search of excellence: Lessons from America's best run companies. New York: Harper \& Row.

Polit, D. F. \& Hungler, B. P. (1987). Nursing research: Principles and methods (3rd ed.). Philadelphia: J. B. Lippincott Co.

Pooyan, A., Eberhardt, B.J., \& Szigeti, E. (1990). Work-related variables and turnover intention among registered nurses. Nursing \& Health Care, 11(5), 255-258.

Porter-O'Grady, T. (1986). Creative nursing administration: Participative management into the 21st century. Rockville, Maryland: Aspen Publications.

Posner, B. Z., Kouzes, J. M., \& Schmidt, W. H. (1985). Shared values make a difference: An empirical test of corporate culture. Human Resource Management, 24(3), 293-309.

Prescott, P.A. and Bowen, S.A. (1987). Controlling nursing turnover. Nursing Management, 18(6), 60-66.

Prestholdt, P. H., Lane, I. M., \& Mathews, R. C. (1988). Predicting staff nurse turnover. Nursing Outlook, 36(3), 145-147.

Raelin, J. A. (1991). The clash of cultures: Managers managing professionals. Boston: Harvard Business School Press.

Ressler, K. A., Kruger, N. R., \& Herb, T. A. (1991). Evaluating a critical care internship program. Dimensions of Critical Care Nursing, 10(3), 176-184.

Roberts, B. L. (1989). Statistics and quantitative methods in medical-surgical nursing. In I. L. Abraham, D. M. Nadzam, \& J. J. Fitzpatrick (Eds.), Statistics and quantitative methods in nursing: Issues and strategies for research and education (p. 52-68). Philadelphia: W. B. Saunders Company.

Roell, S. M. (1981). Nurse-intern programs: How they're working. Journal of Nursing Administration, 11(10), 33-36. 
Rufo, K. (1984). Termination of a successful internship program. Journal of Nursing Administration, 14(6), 33-37.

Sams, L., Baxter, K., \& Palmer-Smith, P. (1990). A competency-based model for nurse internships. Journal of Nursing Staff Development, 6(2), 93-94.

Schaef, A. W. \& Wilson, D. (1988). The addictive organization. San Francisco: Harper \& Row, Publishers.

Senge, P. M. (1990). The fifth discipline: The art and practice of the leaming organization. New York: Doubleday.

Seybolt, J. W. (1986). Dealing with premature employee turnover. Journal of Nursing Administration, 16(2), 26-32.

Schempp, C. M. \& Rompre, R. M. (1986). Transition programs for new graduates: How effective are they? Journal of Nursing Staff Development, 2(4), 150-156.

Shockley-Zalabak, P. \& Morley, D. D. (1989). Adhering to organizational culture: What does it mean? Why does it matter? Group \& Organization Studies, 14(4), 483-500.

Sovie, M. D. (1993). Hospital culture: Why create one? Nursing Economics, 11(2), 69-75.

Speedling, E. J., Ahmadi, K., \& Kuhn-Weissman, G. (1981). Encountering reality: Reactions of newly hired RN's to the world of the medical center. International Journal of Nursing Studies, 18(4), 217-225.

Stevens, J. (1992). Applied multivariate statistics for the social sciences (2nd Ed.). Hillsdale, NJ: Lawrence Erlbaum Associates, Publishers.

Stratton, T. D., Dunkin, J. W., Juhl, N., Ludtke, R. L., Geller, J. M. (1991). Recruiting and retaining registered nurses in rural community hospitals. Journal of Nursing Administration, 21(11), 30-34.

Tabachnick, B. G. \& Fidell, L. S. (1989). Using multivariate statistics (2nd ed.). New York: Harper \& Row, Publishers.

Thomas, C., Ward, M., Chorba, C., \& Kumiega, A. (1990). Measuring and interpreting organizational culture. Journal of Nursing Administration, 20(6), 1724. 
Toothaker, L. E. (1991). Multiple comparisons for researchers. Newbury Park: Sage Publications.

Ullman, J. C. (1987). Understanding values: The key to managing? Business $\underline{\&}$ Economic Review, 33(3), 22-26.

Vanevenhoven, R. A., Stull, M. K., \& Pinkerton, S. (1988). The nursing shortage and staff nurse retention. In M. K. Stull \& S. Pinkerton (Eds.), Current strategies for nurse administrators (pp. 65-80). Rockville, Maryland: Aspen Publications.

Virginia Hospital Association. (1991). Health Manpower: 1921 Survey. Glen Allen, Virginia: Virginia Hospital Association.

Waltz, C. F. \& Bausell, R. B. (1981). Nursing research: Design, statistics, and computer analysis. Philadelphia: F. A. Davis Company.

Weick, K. E. (1979). The social psychology of organizing (2nd ed.). New York: Random House.

Weick, K. E. (1985). Sources of order in underorganized systems: Themes in recent organizational theory. In Y. S. Lincoln (Ed.), Organizational theory and inquiry: The paradigm revolution (p. 106-136). Newbury Park, CA: Sage Publications, Inc.

Weisman, C. S., Alexander, C. S., \& Chase, G. A. (1981). Determinants of hospital staff nurse turnover. Medical Care, 19(4), 431-443.

Weiss, S. J. (1984). The effect of transition modules on new graduate adaptation. Research in Nursing and Health, 7, 51-59.

Weiss, S. J. \& Ramsey, E. (1977). An interagency internship: A key to transitional adaptation. Journal of Nursing Administration, $\underline{7}(8), 36-42$.

Whaley, B. A., Young, W. B., Adams, C. J., \& Biordi, D. L. (1989). Targeting recruitment efforts for increased retention. Journal of Nursing Administration, 19(4), 34-38.

Winer, B. J. (1971). Statistical principles in experimental design (2nd ed.). New York: McGraw-Hill Book Company. 
Appendix A

Consent Form 


\section{CoVenture Nurse Transition Program}

All new graduates entering employment in Nursing Services at Pitt County Memorial Hospital are asked to complete the attached battery of research instruments. The purpose of this research study component is to collect baseline data on role transition from student to health care provider. Findings from this study will be used to help develop more effective ways to ease transition and to possibly combat the onset of "reality shock". Both nursing and education programs and agency nursing services will have access to the results, so you will by your participation, not only assist in "bridging the gap," but also in the search for a model to promote healthy adjustment to the work world.

Included in the battery are:

1. Profile sheet - contains questions on personal and demographic variables

2. Role Function Survey - which measures role expectations

3. Nursing Characteristics Index - records perception of nursing practice

4. Competency Self-assessment

Please circle one response for each item, unless otherwise indicated. If you have any questions, please do not hesitate to ask. The information is confidential, recorded by number only.

At a later time (3-4 weeks) you will be asked to complete a job satisfaction tool. This will be readministered with survey tool at 6 and 12 months.

We appreciate your thoughtful and completely candid self appraisal and ratings. Please do not hesitate to address questions or concerns regarding the CoVenture Program to the Director of Nurse Transition or you Educational Nurse Specialist.

\section{Participant consent form}

I have read the above description of the CoVenture Project and understand my participation during the next year.

Signature of Nurse

Project Number

Date

Group Initial 6 months 12 months 
Appendix B

Clinical and Seminar Competencies 


\section{CLINICAL COMPETENCY STATEMENTS}

\section{$\underline{\text { Assessment }}$}

Assesses the patient's/family's perception of health status and limitations.

Assesses the present status and pertinent past history of: cardiovascular function; respiratory function; skin integument function; neurological function; psychosocial function.

Assesses and incorporates deviations from normal into nursing diagnoses, develops and maintains nursing care plans.

\section{Intervention}

Uses assessment data and medical therapies for assigned patients to determine and prioritize the interventions to be carried out.

Plans equipment to be used and organizes work in order to carry out all necessary procedures.

Demonstrates the ability to carry out procedures according to the procedure manual.

Evaluates the procedures done and documents appropriately.

\section{Medications}

Assesses the actions, side effects, and dosage of the patient's prescribed medication and rationale for their use.

Demonstrates steps of preparing, administering, and charting medications according to the hospital's policies and procedures.

Identifies patient's reaction to medications and communicates and documents appropriately.

\section{Documentation}

Assesses the information to be documented and gives rationale for the documentation.

Documents the information on the appropriate form.

Documentation reflects the nursing care plan, provides information for other care givers, increases the data base, indicates change in the patient's status, and is legally sound. 


\section{$\underline{\text { Risk Management }}$}

Assesses the patient's environment to provide for safe patient care.

Utilizes proper lifting and moving techniques to protect her/himself and the patient.

Reports and documents incidents in a timely and accurate manner.

\section{Patient Support}

Assesses the patient's feelings about his hospitalization and sense of personal worth.

Assists the patient to maintain his sense of personal identity and self worth by listening and providing emotional support; by answering questions; by respecting the patient's privacy.

Assesses and provides for patient's rest and comfort needs.

Assesses and provides for patient's nutritional needs.

Assesses the patient's leaming needs: plans with patient/family and members of health care team to meet educational needs; provides knowledge and skills related to health status, procedures performed, coping with health status limitations, and community resources. 


\section{SEMINAR COMPETENCIES AND OBJECTIVES}

\section{$\underline{1983-1987}$}

"Getting Into The Group." Establishes self as a productive group member within the roles and functions of the group.

Defines the process of socialization into a small group.

Formulates strategies to obtain group membership.

"Using Clinical Judgment." Utilizes the nursing process in making clinical judgments regarding individual patients and/or groups of patients.

Identifies the relationship between nursing process and clinical judgment.

Identifies resources for direct and indirect assessment of the patient.

Identifies personal and situational factors influencing judgment.

Formulates strategies for handling situations involving individual patient and/or groups of patients.

"Selecting Priorities." Utilizes a model for making decisions in multiple-priority, fastaction situations.

Identifies factors influencing selecting priorities.

Identifies strategies for making judgments about priorities.

"Coping With The New Role." Establishes realistic, effective nursing role for self.

Verbalizes own ideal professional nursing role.

Identifies unrealistic role expectations of self and others.

Identifies past and/or current roles which impinge on nursing role.

Considers role expectations of others.

Blends components of the ideal role with reality for the development of an effective nursing role for self.

"Coping With Others." Understands and uses the resources of others.

Utilizes fellow workers' behaviors to formulate a hypothesis about their feeling state in order to communicate more effectively.

Uses behavioral approaches to interpersonal situations which facilitate individual growth and patient care. 
"Working Together." Analyzes the performance needs of others.

Observes behavior to determine when there is a performance problem.

Evaluates data to determine if the problem is important and requires a solution.

Obtains and analyzes data to determine the probable cause of the performance problem.

Determines a realistic solution based on individual differences and available options.

"Understanding The Hospital System." Understands the hospital as a business system.

Identifies the external and internal economic constraints.

Explains how cost containment effects nursing and the individual nurse.

Defines the staff nurse's responsibility for cost containment.

Understands the decision making process within the hospital.

Understands and utilizes the formal and informal communication systems.

"Blending Independence And The System." Meets individual need for professional fulfillment within hospital parameters.

Identifies own needs for satisfaction as a nurse.

Delineates specific barriers which inhibit need satisfaction.

Identifies strategies for coping with barriers to need satisfaction.

Identifies strategies for coping with barriers which are beyond individual resolution.

$\underline{1988-1992}$

"Transition Workshop." The goal of the seminar is to assist the new nurse in decision making and adjustment to the RN role.

Determines practical approaches to clinical judgment using Human Need Theory.

Develops strategies for setting priorities to satisfy unmet needs.

Identifies effective coping skills in adapting to the $\mathrm{RN}$ role.

Identifies stress management strategies to utilize with issues facing the new RN.

Delineates tasks appropriate to skill level of other caregivers.

Analyzes performance problems in self and others.

Identifies ethical issues and approaches for resolution. 
$\because$

153

Appendix C

Data Collection Instruments 
PROFILE DATA SHEET

Unless otherwise indicated, circle the one best response.

1. Your age is:
a) under 24 years
b) 25-29 years
c) 30-34 years
d) 35-39 years
e) 40 years and above

2. Sex:
a) Female
b) Male

3. Marital status:
a) Single
b) Married less than 1 year
c) Married more than 1 year
d) Separated
e) Divorced

4. Basic nursing education program:
a) Associate Degree
b) Diploma
c) Baccalaureate Degree
d) Other

5. What is the highest degree you expect to receive in your career?
a) B.S. in nursing
b) Masters in Nursing
c) Masters in another field
d) Doctorate in Nursing
e) Other Doctorate 
6. Which of the following describes your employment status while in school?
a) Full-time employment
b) Part-time employment
c) Summer employment only
d) None
e) Other

7. Was employment in \#6 some type of nursing?
a) Yes
b) No
c) Not applicable

8. Which experience(s) have you had in the hospital setting? (Circle as many as apply)
a) Nursing Assistant
b) LPN
c) Nurse Extern (at this hospital)
d) Nurse Extern (other than this hospital)
e) Other, please describe

9. What is your greatest area of interest in clinical practice?
a) Intensive Care/Emergency Nursing
b) Medical Nursing
c) Surgical Nursing
d) Pediatric Nursing
e) Maternity Nursing
f) Astrchliatrian
d) Always
e) Other than above (describe) 
11. Why have you chosen to work at this hospital? (Circle one)
a) Familiar territory
b) Good pay
c) Opportunity to continue education
d) Close to home
e) Developing medical center
f) Close to boy/girl friend/spouse
g) Positive work environment
h) Other

12. Which school did you attend?

[The schools listed for this item are omitted to protect the anonymity of the hospital study site; data from this item are not included in this study's analyses] 


\section{NURSING COMPETENCY ASSESSMENT}

This section lists nursing competencies and subcompetencies which are assessed in the transition program.

A. These are competencies which are performed in general nursing practice. Please indicate the level at which you are presently performing these competencies.

Scale for Level of Performance

$5=$ High Proficiency

$4=$ Moderate Proficiency

3 = Safe, Practice Needed

2 = Supervision Needed

$1=$ Supervision and

Instruction Needed

Able to perform competently and efficiently without supervision.

Able to perform without supervision with reasonable proficiency.

Able to perform without supervision but more practice is needed in order to perform efficiently.

Understand theory and principles, but would need supervision because of limited practice experience.

Have not been introduced to the theory and principles and need both instruction and supervision to complete this competency.

\section{$\underline{\text { ASSESSMENT }}$}

1. Assess patient/family perceptions of health status and limitations

-- Assess the present health status and pertinent health history of:

2. Cardiovascular function $\begin{array}{lllll}1 & 2 & 3 & 4 & 5\end{array}$

3. Respiratory function

$\begin{array}{lllll}1 & 2 & 3 & 4 & 5\end{array}$

4. Skin integument function $\begin{array}{lllll}1 & 2 & 3 & 4 & 5\end{array}$

5. Neurologic function

$\begin{array}{lllll}1 & 2 & 3 & 4 & 5\end{array}$


6. Psychosocial function

7. Assess and incorporate deviations from normal into nursing diagnoses, develop and maintain care plans

\section{INTERVENTION}

8. Use assessment data and medical therapies for assigned patients to determine and prioritize interventions

9. Plan equipment to be used and organize work in order to carry out procedures

10. Carry out procedures according to Procedure Manual

11. Evaluate procedures done and document appropriately

\section{MEDICATIONS}

12. Assess actions, side effects and dosage of patient medication and describe rationale for use

13. Identify patient reactions to medications, communicate and document appropriately

\section{DOCUMENTATION}

14. Assess information to be documented and rationale for documentation (e.g., know critical elements for common patient situations on unit-pre-op/neuro/ chemotherapy/cardiac)

15. Utilize proper forms for documentation (e.g., nurses notes, med sheets) $\begin{array}{lllll}1 & 2 & 3 & 4 & 5\end{array}$

$\begin{array}{lllll}1 & 2 & 3 & 4 & 5\end{array}$

$\begin{array}{lllll}1 & 2 & 3 & 4 & 5\end{array}$

$\begin{array}{lllll}1 & 2 & 3 & 4 & 5\end{array}$

$\begin{array}{lllll}1 & 2 & 3 & 4 & 5\end{array}$

$\begin{array}{lllll}1 & 2 & 3 & 4 & 5\end{array}$

$\begin{array}{lllll}1 & 2 & 3 & 4 & 5\end{array}$

$\begin{array}{lllll}1 & 2 & 3 & 4 & 5\end{array}$

$\begin{array}{lllll}1 & 2 & 3 & 4 & 5\end{array}$

$\begin{array}{lllll}1 & 2 & 3 & 4 & 5\end{array}$ 
16. Document so it reflects the nursing care plan, provide information for

1

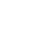

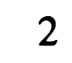

3

4

5 other caregivers, increase the data base, indicate change in patient status and is legally sound

\section{RISK MANAGEMENT}

17. Assess patient environment to provide for safe patient care (e.g., comfort, safety, infection control)

18. Utilize proper lifting and moving techniques to protect self/patient

19. Report and document incidents in a timely and accurate manner

$\begin{array}{lllll}1 & 2 & 3 & 4 & 5\end{array}$

$\begin{array}{lllll}1 & 2 & 3 & 4 & 5\end{array}$

$\begin{array}{lllll}1 & 2 & 3 & 4 & 5\end{array}$

\section{PATIENT SUPPORT}

20. Assess patient's feelings about hospitalization and sense of worth

21. Assist patient to maintain sense of personal identity and self worth by listening, provide emotional support, answer questions, respect privacy

22. Assess and provide patient rest/comfort needs

23. Assess and provide for patient nutritional needs

24. Assess patient leaming needs and provide patient/family teaching

$\begin{array}{lllll}1 & 2 & 3 & 4 & 5 \\ 1 & 2 & 3 & 4 & 5\end{array}$

$\begin{array}{lllll}1 & 2 & 3 & 4 & 5\end{array}$

$\begin{array}{lllll}1 & 2 & 3 & 4 & 5\end{array}$

$\begin{array}{lllll}1 & 2 & 3 & 4 & 5\end{array}$

B. Professionalization

The following items relate to your level of functioning in areas of your professional development.
25. Establish self as productive group
12
3
4
member in practice area 
26. Establish realistic, effective nursing role for self

27. Understand and utilize the resources of fellow workers

28. Analyze the performance needs of others

29. Understand the hospital as a business system (how it affects nursing, constraints, decision making process)

30. Meet own need for professional fulfillment within the hospital employment situation (identify barriers, develop strategies, etc.) $\begin{array}{lllll}1 & 2 & 3 & 4 & 5\end{array}$

$\begin{array}{lllll}1 & 2 & 3 & 4 & 5\end{array}$

$\begin{array}{lllll}1 & 2 & 3 & 4 & 5\end{array}$

$\begin{array}{lllll}1 & 2 & 3 & 4 & 5\end{array}$

$\begin{array}{lllll}1 & 2 & 3 & 4 & 5\end{array}$




\section{NURSING CHARACTERISTICS SCALE}

Please indicate your level of agreement on the following list of nursing characteristics.

Your responses will range on a 1 to 5 scale from strongly disagree (1), disagree (2), undecided (3), agree (4), to strongly agree (5).

\section{NURSING-}

1 . is a very respected occupation in our society

2. provides many opportunities for advancement

3. allows me to avoid working under very high pressure

4. fits my abilities and skills; lets me do things I can do best

5. fits my interests; something I really like

$\begin{array}{lllll}1 & 2 & 3 & 4 & 5\end{array}$

6. gives me a chance for leadership and responsibility

7. gives me a chance to help others; be useful to society

8. allows me to be creative and original

$\begin{array}{lllll}1 & 2 & 3 & 4 & 5\end{array}$

9. promises a secure future

$\begin{array}{lllll}1 & 2 & 3 & 4 & 5\end{array}$

10. brings a high income

$\begin{array}{lllll}1 & 2 & 3 & 4 & 5\end{array}$

11. leaves me relatively free of supervision by others

12. deals primarily with people rather than things

$\begin{array}{lllll}1 & 2 & 3 & 4 & 5\end{array}$


13. allows me time to pursue other interests 12

3

4 5 (hobbies, family, etc.)

14. gives me a chance to help bring out needed 1 changes

$\begin{array}{llll}2 & 3 & 4 & 5\end{array}$




\section{STAFF NURSE ROLE FUNCTION SURVEY}

The following items describe role expectations which are part of your position as a staff nurse. These represent competencies which develop over time as you become familiar with the work demands and environment. Please rate your present level of confidence on each of the areas described below.

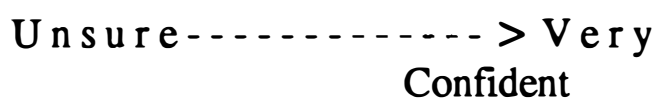

1. Utilize the nursing process in giving patient care

2. Able to accurately and consistently document care on proper charts and forms

3. Familiar with care protocols relating to clinical area

4. Familiar with unit policies and procedures

5. Familiar with hospital philosophy and objectives

6. Identify hospital resources both human and material which contribute to care delivery

7. Use hospital resources appropriately

8. Feel comfortable as part of unit work group

9. Adjust to time schedule and demands of staffing pattern

10. Set own personal goals for continued growth and professional development

11. Assess leaming needs and seek out educational opportunities

12. Understand charge nurse responsibilities
A B
C
D E

A $B \quad$ C $\quad$ D $\quad$ E

A $B \quad$ C $\quad$ D $\quad$ E

A $\quad$ B $\quad$ C $\quad$ D $\quad$ E

A $B \quad$ C $\quad$ D $\quad$ E

A $\quad$ B $\quad$ C $\quad$ D $\quad$ E

A $\quad$ B $\quad$ C $\quad$ D $\quad$ E

A $\quad$ B $\quad$ C $\quad$ D $\quad$ E

A $\quad$ B $\quad$ C $\quad$ D $\quad$ E

A $B \quad$ C $\quad$ D $\quad$ E

A $B \quad$ C D

A $B \quad$ C D 
13. Supervise and direct ancillary care personnel

A $\quad$ B $\quad$ C $\quad$ D $\quad$ E

14. Work with the organizational and bureaucratic system

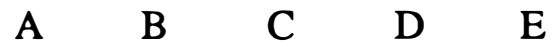

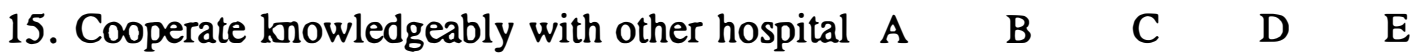
services and departments

16. Demonstrate good communications skills

$\begin{array}{lllll}\text { A } & \text { B } & \text { C } & \text { D } & \text { E }\end{array}$

17. Deal assertively with conflict situations

$\begin{array}{lllll}\text { A } & \text { B } & \text { C } & \text { D } & \text { E }\end{array}$

18. Relate collegially with the medical staff

A $\quad$ B $\quad$ C $\quad$ D $\quad$ E

19. Cope constructively with work stress

$\begin{array}{lllll}\text { A } & \text { B } & \text { C } & \text { D } & \text { E }\end{array}$

20. Solve most work related problems as they arise

$\begin{array}{lllll}\text { A } & \text { B } & \text { C } & \text { D } & \text { E }\end{array}$

21. Feel satisfied with working conditions

$\begin{array}{lllll}\text { A } & \text { B } & \text { C } & \text { D } & \text { E }\end{array}$ 


\section{JOB SATISFACTION INDEX}

How satisfied are you with the following job variables?
(A) Dissatisfied
(B) Less satisfied
(C) Middle range satisfied
(D) Satisfied
(E) Very Satisfied

Dissatisfied------------> Very Satisfied

1. Prestige inside the hospital

2. The opportunity to fully use my skills and abilities

3. The opportunity to do important and worthwhile things

4. A feeling of self-fulfillment

5. A sense of pressure

6. Financial rewards

7. Job security

8. Fair working conditions

9. The opportunity to exercise independent judgment

10. Working with likable people

11. Understanding by supervisors of my job problems

12. Understanding by others of my job problems

13. The opportunity to give help to others

14. Sharing in the determination of methods and procedures
A $\quad$ B $\quad$ C $\quad$ D $\quad$ E

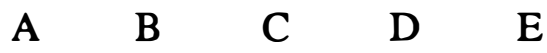

A $\quad$ B $\quad$ C $\quad$ D $\quad$ E

A $\quad$ B $\quad$ C $\quad$ D $\quad$ E

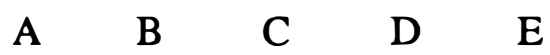

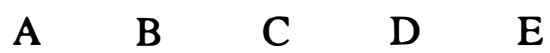

A $\quad$ B $\quad$ C $\quad$ D $\quad$ E

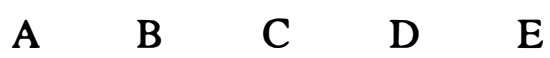

A $\quad$ B $\quad$ C $\quad$ D $\quad$ E

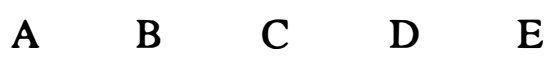

A $\quad$ B $\quad$ C $\quad$ D $\quad$ E

$\begin{array}{lllll}A & B & C & D & E\end{array}$

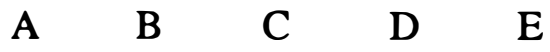

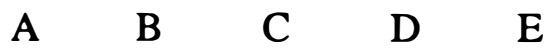


15. Having authority to direct others

A $B \quad$ C $\quad$ D $\quad$ E

16. Sharing in the setting of goals

A $B \quad$ C $\quad$ D $\quad$ E 